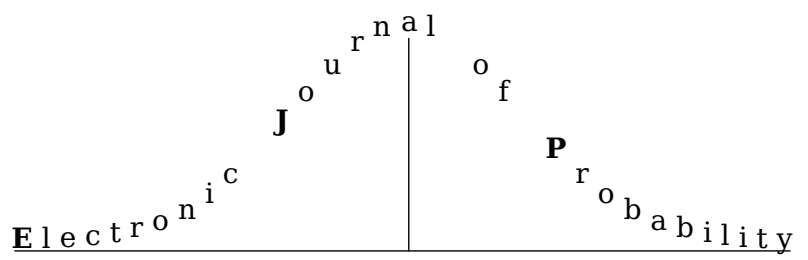

Electron. J. Probab. 26 (2021), article no. 145, 1-49.

ISSN: 1083-6489 https://doi.org/10.1214/21-EJP700

\title{
Priors leading to well-behaved Coulomb and Riesz gases versus zeroth-order phase transitions - a potential-theoretic characterization
}

\author{
Robert J. Berman*
}

\begin{abstract}
We give a potential-theoretic characterization of measures $\mu_{0}$ which have the property that the Coulomb gas, defined with respect to the prior $\mu_{0}$, is "well-behaved" and similarly for more general Riesz gases. This means that the laws of the empirical measures of the corresponding random point process satisfy a Large Deviation Principle with a rate functional which depends continuously on the temperature, in the sense of Gamma-convergence. Equivalently, there is no zeroth-order phase transition at zero temperature, in the mean field regime. This is shown to be the case for the Hausdorff measure on a compact Lipschitz hypersurface, as well as Lesbesgue measure on a bounded Lipschitz domain. We also provide constructions of priors $\mu_{0}$, absolutely continuous with respect to Lebesgue measure on a smoothly bounded domain, such that the corresponding $2 \mathrm{~d}$ Coulomb exhibits a zeroth-order phase transition. This is based on relations to Ullman's criterion in the theory of orthogonal polynomials and Bernstein-Markov inequalities.
\end{abstract}

Keywords: statistical mechanics type models; large deviations; phase transitions; fine potential theory.

MSC2020 subject classifications: $60 \mathrm{~K} 35 ; 60 \mathrm{~F} 10$; 82B26; 31C40.

Submitted to EJP on March 31, 2020, final version accepted on September 7, 2021.

\section{Introduction}

In broad terms, the main aim of the present work is to study the interplay between fine potential-theoretic properties of a measure $\mu_{0}$ in $\mathbb{R}^{d}$ (the "prior") and properties of the corresponding Coulomb gas in $\mathbb{R}^{d}$, in the mean-field regime. The pair-interaction potential of this gas (also known as the one-component plasma in the physics literature) is the fundemental solution of the Laplacian $\Delta$ in $\mathbb{R}^{d}$ and yields a family of random point processes on the support of $\mu_{0}$ in $\mathbb{R}^{d}$, parametrized by temperatures $\left.\left.T_{N} \in\right] 0, \infty\right]$. We will, in particular, give a potential-theoretic characterization of measures $\mu_{0}$ for which the

${ }^{*}$ E-mail: robertb@chalmers.se 
corresponding Coulomb gas is "well-behaved at zero-temperature", which is equivalent to the absence of a zeroth-order phase transition. In fact, the main results will be shown to hold in a more general setting involving a Riesz gas in $\mathbb{R}^{d}$, where the role of the Laplacian is played by the fractional Laplacian $-(-\Delta)^{\alpha / 2}$, assuming that $\left.\left.\alpha \in\right] 0,2\right]$. Complex-geometric analogs of the results, where the role of the Laplacian is played by the complex Monge-Ampère operator, are described in [9]. Our results yield new probabilistic constructions of capacities, equilibrium measures etc, using random point processes, in contrast to the usual probabilistic approach based on Markov processes (and their hitting probabilities [33, 22]). More precisely, the setting of a Riesz gas with $\alpha \in] 0,2]$ corresponds precisely to the class of symmetric stable Levy processes in $\mathbb{R}^{d}$ [20] (i.e. Brownian motion in the "Coulomb case" $\alpha=2$ ).

For concreteness we will introduce the main results in the Coulomb case $(\alpha=2)$, where the energy $E(\mu)$ of a compactly supported measure $\mu$ in $\mathbb{R}^{d}$, with $d \geq 2$, is defined by

$$
E(\mu)=\frac{1}{2} \int_{\mathbb{R}^{d}} W \mu \otimes \mu,
$$

where $W(x, y)$ denotes the standard Green function of the Laplacian $\Delta$, i.e. $W(x, y)$ is proportional to $|x-y|^{2-d}$ when $d \geq 3$ and to $-\log |x-y|$ when $d=2$. The potential $\psi_{\mu}$ of $\mu$ is the subharmonic function on $\mathbb{R}^{d}$ defined by

$$
\psi_{\mu}(x):=-\int_{\mathbb{R}^{d}} W(x, y) \mu(y)
$$

(using the opposite sign convention compared to the standard convention in physics). A bounded subset $S$ of is said to be polar if there exists a potential $\psi_{\mu}$ such that $S \Subset\left\{\psi_{\mu}=-\infty\right\}$. We will be particularly interested in measures $\mu_{0}$ not charging polar subsets (for example, this is the case if $\mu_{0}$ has finite energy or if $\mu_{0}$ is absolutely continuous wrt Lebesgue measure). We will denote by $\mathcal{P}(S)$ the space of all probability measures on a closed subset $S \subset \mathbb{R}^{d}$, endowed with the weak topology.

\subsection{Energy approximation and determining measures}

The main analytical result may be formulated in terms of potential theory and approximation theory as follows. Assume given a measure $\mu_{0}$ on $\mathbb{R}^{d}$ and denote by $S_{0}$ its support. We will say that $\mu_{0}$ has the Energy Approximation Property if for any measure $\mu$ supported on $S_{0}$ there exists a sequence $\mu_{j}$ converging weakly towards $\mu$ such that

- $\mu_{j}$ is absolutely continuous with respect to $\mu_{0}$

- $\lim _{j \rightarrow \infty} E\left(\mu_{j}\right)=E(\mu)$

Note that, by the lower semi-continuity of $W$, the second point is equivalent to

$$
\limsup _{j \rightarrow \infty} E\left(\mu_{j}\right) \leq E(\mu)
$$

Theorem 1.1 below relates the Energy Approximation Property to the potential-theoretic notion of determining measures. Given a weighted set $(S, \phi)$ consisting of a subset $S$ of $\mathbb{R}^{d}$ and a continuous function $\phi$ on $S$, a measure $\nu$ on $\mathbb{R}^{d}$ is said to be determining for $(S, \phi)$ if for all potentials $\psi$ on $\mathbb{R}^{d}$

$$
\psi \leq \phi \text { almost everywhere wrt } \nu \Longrightarrow \psi \leq \phi \text { on } S
$$

We will say that $\nu$ is determining for $S$ if $\nu$ is determining for $(S, 0)$ and strongly determining for $S$ if $\nu$ is determining for $(S, \phi)$ for all $\phi \in C(S)$. Similarly we will say that $\nu$ is (strongly) determining if it is (strongly) determining for its support. For example, 
Priors leading to well-behaved Coulomb, Riesz gases vs zeroth-order phase transitions

Lebesgue measure $1_{\Omega} d x$ on a bounded domain $\Omega$ in $\mathbb{R}^{d}$ is strongly determining if $\Omega$ is nonthin at all boundary points, in the classical sense (Prop 2.21). In general, if $\nu$ is strongly determining and does not charge polar subsets then the support of $\nu$ is automatically locally regular (see Section 2.7 for the potential-theoretic notions of regularity).

Theorem 1.1. Let $\mu_{0}$ be a measure on $\mathbb{R}^{d}$ which does not charge polar subsets and assume that the support $S_{0}$ of $\mu_{0}$ is compact and locally regular. Then $\mu_{0}$ has the Energy Approximation Property iff $\mu_{0}$ is strongly determining.

The main virtue of the property of beeing strongly determining is that it can often be verified using maximum (/domination) principle type arguments. For example, using [27], we will show that the $(d-1)$-dimensional Hausdorff measure on a Lipschitz hypersurface is strongly determining (Theorem 2.22).

Theorem 1.1 appears to be new even in the simplest case when $S_{0}$ is an interval in $\mathbb{R} \subset \mathbb{R}^{2}$, which is the classical setting where the notion of determining measures was first introduced by Ullman (as discussed in Section 1.3 below). In this special case a measure $\mu_{0}$ is determining iff it is strongly determining.

\subsection{The zero-temperature limit of the Coulomb gas in the mean-field regime}

The main motivation for Theorem 1.1 above comes from the study of the large deviations of the Coulomb gas on a measure $\mu_{0}$ in $\mathbb{R}^{d}$, where the Energy Approximation Property has previously appeared as a technical hypothesis [26, 29, 7, 32]. To give some background, assume given a continuous function $\phi$ on $\mathbb{R}^{d}$ and consider the corresponding mean field $N$-particle Hamiltonian

$$
H_{\phi}^{(N)}\left(x_{1}, \ldots x_{N}\right):=\frac{1}{(N-1)} \frac{1}{2} \sum_{i \neq j} W\left(x_{i}, x_{j}\right)+\sum_{i=1}^{N} \phi\left(x_{i}\right)
$$

describing the Coulomb energy of $N$-particles in the exterior potential $\phi$ (with the divergent self-energies removed) with a mean field scaling. In physical terms this means that each particle is subject to the average of the Coulomb potentials created by the other particles, plus the exterior potential $\phi$. We recall that the mean-field scaling is often used for systems with long-range interactions, i.e. such that $W(x, y) \leq C 1 /|x-y|^{d}$ (which includes general Riesz gases) [3, 23, 40]. Given a measure $\mu_{0}$ on $\mathbb{R}^{d}$ (the "prior") with compact support $S_{0}$ and a sequence of numbers $\left.T_{N} \in\right] 0, \infty[$ the corresponding (mean field) Coulomb gas at temperature $T_{N}$ on $\mu_{0}$ is defined as the probability space (canonical ensemble) $\left(\left(\mathbb{R}^{d}\right)^{N}, \mu_{T_{N}}^{(N)}\right)$, where

$$
\mu_{\phi, T_{N}}^{(N)}:=\frac{1}{Z_{N, \phi, T_{N}}} e^{-T_{N}^{-1} H_{\phi}^{(N)}} \mu_{0}^{\otimes N}, \quad Z_{N, \phi, T_{N}}:=\int_{\left(\mathbb{R}^{d}\right)^{N}} e^{-T_{N}^{-1} H_{\phi}^{(N)}} \mu_{0}^{\otimes N}
$$

The normalizing constant $Z_{N, \phi, T_{N}}$ is called the partition function and

$$
F_{N, \phi, T_{N}}:=-\frac{T_{N}}{N} \log Z_{N, \phi, T_{N}}
$$

is called the $N$-particle free energy at temperature $T_{N}$. It extends continuously to $T_{N}=0$ by setting

$$
F_{N, \phi, 0}:=\frac{1}{N} \inf _{S_{0}^{N}} H_{\phi}^{(N)}
$$

where $S_{0}$ is the support of $\mu_{0}$ (using that $e^{-H_{\phi}^{(N)}}$ is continuous; see the beginning of the proof of Lemma 5.3). In the case when $\phi=0$ we will simply drop the subscripts 
$\phi$. The mean field scaling $1 /(N-1)$ appearing in the definition of $H_{\phi}^{(N)}\left(x_{1}, \ldots x_{N}\right)$ could alternatively have been absorbed by the temperature $T_{N}$. Anyhow, throughout the paper we will employ the mean field scaling above and assume that the corresponding temperatures $T_{N}$ have a limit $T$ as $N \rightarrow \infty$ :

$$
T:=\lim _{N \rightarrow \infty} T_{N} \in[0, \infty[.
$$

For example, if, with the present mean field scaling, $T_{N}$ is taken to be proportional to $1 /(N-1)$, so that $\mu_{T_{N}}^{(N)}$ is the Gibbs measure for a Coulomb gas without a mean field scaling at a fixed positive temperature [52], then the corresponding limiting temperature $T$ above vanishes (compare Section 1.3). But here it will be important to allow nonvanishing limiting temperatures $T$, where entropy enters the picture.

As first shown in $[23,41]$, in the case when $T>0$ and $\mu_{0}$ is equal to Lebesgue measure on a compact domain $S_{0}$, the empirical measure

$$
\delta_{N}:=\frac{1}{N} \sum_{i=1}^{N} \delta_{x_{i}},
$$

viewed as a random measure on $\left(\left(\mathbb{R}^{d}\right)^{N}, \mu_{T_{N}}^{(N)}\right)$, converges in probability, as $N \rightarrow \infty$, towards a deterministic measure $\mu_{\phi, \beta}$

$$
\lim _{N \rightarrow \infty} \delta_{N}=\mu_{\phi, T}
$$

where $\mu_{\phi, T}$ is the unique minimizer of the following free energy functional $F_{\phi, T}$ on the space $\mathcal{P}\left(S_{0}\right)$ :

$$
F_{\phi, T}(\mu)=E_{\phi}(\mu)+T D_{\mu_{0}}(\mu), \quad E_{\phi}(\mu):=E(\mu)+\int \phi \mu
$$

where $D_{\mu_{0}}$ denotes the entropy of $\mu$ relative to $\mu_{0}$, using the sign convention making $D_{\mu}$ non-negative (see formula (3.2)). In particular,

$$
F_{\phi, 0}:=E_{\phi}
$$

and we denote by $\mu_{(S, \phi)}$ the equilibrium measure of a non-polar compact weighted set $(S, \phi)$, i.e. the unique minimizer of $E_{\phi}$ on $\mathcal{P}(S)$. It should be stressed that the presence of the entropy term in the free energy $F_{\phi, T}(\mu)$ entails that the minimizer $\mu_{T}$ is absolutely continuous wrt $\mu_{0}$ for any $T>0$, while this is often not the case for the equilibrium measure $\mu_{\left(S_{0}, \phi\right)}$. For example, if $\mu_{0}$ is Lesbesgue measure on a compact domain $S_{0}$ with smooth boundary and $\phi=0$, then $\mu_{\left(S_{0}, \phi\right)}$ is supported in the boundary of $S_{0}$ (in the Coulomb case).

Under appropriate regularity assumptions on $\mu_{0}$ it is shown in $[26,29,7,32]$ that the convergence of $\delta_{N}$ towards $\mu_{\phi, T}$ is, in fact, exponential in the sense of large deviation theory. More precisely, the laws of the empirical measures $\delta_{N}$ satisfy a Large Deviation Principle ( $L D P$ ) at speed $T_{N}^{-1} N$, whose rate functional $I_{\phi, T}$ coincides with $F_{\phi, T}$, up to an additive constant. In symbolic notation this may be expressed as

$$
\left(\delta_{N}\right)_{*}\left(e^{-T_{N}^{-1} H_{\phi}^{(N)}} \mu_{0}^{\otimes N}\right) \sim e^{-T_{N}^{-1} N F_{\phi, T}}, N \rightarrow \infty
$$

as measures on $\mathcal{P}\left(S_{0}\right)$. We recall that, in general, the rate functional $I$ for an LDP is a proper lower-semicontinuous function (the precise meaning of the LDP is recalled in Section 4). 
Priors leading to well-behaved Coulomb, Riesz gases vs zeroth-order phase transitions

\subsubsection{The LDP in the zero-temperature limit}

When $T>0$ the LDP for the Coulomb gas holds for any measure $\mu_{0}$ not charging polar subsets (Theorem 4.2). A natural question is thus what further conditions on $\mu_{0}$ need to be imposed in order to ensure that the LDP also holds for $T=0$ ? As shown in [26, 29, 7, 32], the Energy Approximation Property is a sufficient condition. However, as will be shown below, this condition is not necessary, but rather equivalent to a "wellbehaved" LDP. The starting point is the basic observation that the Energy Approximation Property is equivalent to a certain continuity property of the free energy functional $F_{T}$, namely that $F_{T}$ be continuous with respect to Gamma-convergence of functionals, as $T \rightarrow 0$. Recall that the notion of Gamma-convergence plays a prominent role in variational calculus (see definition 3.1) and corresponds to the Fell topology on the space of lower-semicontinuous functions [24]. It implies, in particular, that the minimizer $\mu_{T}$ of $F_{T}$ converges towards the minimizer of $F_{0}$. In the present setting the Gammaconvergence of the functional $F_{T}$ is, by a duality argument, equivalent to the continuity of

$$
f_{\phi}(T):=\inf _{\mu \in \mathcal{M}\left(S_{0}\right)} F_{\phi, T}(\mu)
$$

as $T \rightarrow 0$ for all exterior potentials $\phi$. The number $f_{\phi}(T)$ is usually called the free energy at temperature $T$ (wrt the exterior potential $\phi$ ). The main result may now be formulated as the following

Theorem 1.2. Let $\mu_{0}$ be a measure on $\mathbb{R}^{d}$ which does not charge polar subsets and assume that the support $S_{0}$ of $\mu_{0}$ is compact and locally regular. Then the following is equivalent:

- $\mu_{0}$ is strongly determining

- For any given continuous function $\phi$, the corresponding free energy $f_{\phi}(T)$ at temperature $T$,

$$
f_{\phi}(T):=\inf _{\mu \in \mathcal{M}\left(S_{0}\right)} F_{\phi, T}(\mu)
$$

is continuous wrt $T \in[0, \infty]$

- For any given potential $\phi$ the minimizer $\mu_{\phi, T}$ of the functional $F_{T, \phi}$ on $\mathcal{P}\left(S_{0}\right)$ converges, as $T \rightarrow 0$, towards the equilibrium measure of $\left(S_{0}, \phi\right)$, i.e. towards the minimizer $\mu_{\left(S_{0}, \phi\right)}$ of $F_{\phi, 0}$ :

$$
\lim _{T \rightarrow 0} \mu_{\phi, T}=\mu_{\left(S_{0}, \phi\right)}
$$

in the weak topology on $\mathcal{P}\left(S_{0}\right)$.

- The LDP for the Coulomb gas on $\mu_{0}$ holds for all exterior potentials $\phi$ and all $T \in[0, \infty[$ with a rate functional which is continuous wrt $T \in[0, \infty[$ in the sense of Gamma-convergence

In fact, in the present setting the LDP in the previous theorem is equivalent to the free energy asymptotics

$$
\lim _{N \rightarrow \infty} F_{N, \phi, T_{N}}=f_{\phi}(T), \quad T_{N} \rightarrow T,
$$

for any potential $\phi$ and sequence $T_{N} \in\left[0, \infty\left[\right.\right.$ such that $T_{N} \rightarrow T \in[0, \infty[$ for a function $f_{\phi}(T)$ which is continuous on $[0, \infty[$ (and a posteriori of the form (1.5)). The continuity of $f_{\phi}(T)$ when $T>0$ is automatic and, as discussed in Section 5, a discontinuity at $T=0$ can be interpreted as a zeroth-order phase transition. Another equivalent formulation is obtained by taking $\left.T_{N}=T \in\right] 0, \infty[$ and demanding that the limits $T \rightarrow 0$ and $N \rightarrow \infty$ of 
Priors leading to well-behaved Coulomb, Riesz gases vs zeroth-order phase transitions

$F_{N, \phi, T}$ commute (see Lemma 5.3). The existence of discontinuities at $T=0$ should be contrasted with the fact that

$$
\lim _{T \rightarrow \infty} \mu_{\phi, T}=\mu_{0}
$$

always holds (see Section 3.4). This means that if $\mu_{0}$ is strongly determining, then the curve $\mu_{T, \phi} \in \mathcal{P}\left(S_{0}\right)$ interpolates between $\mu_{0}$ at $T=\infty$ and the weighted equilibrium measure $\mu_{\left(S_{0}, \phi_{0}\right)}$ at $T=0$.

The previous theorem will be deduced from the following result concerning the case when the potential $\phi$ is fixed:

Theorem 1.3. Let $\mu_{0}$ be a measure on $\mathbb{R}^{d}$ which does not charge polar subsets and assume that the support $S_{0}$ of $\mu_{0}$ is compact and locally regular. For a given continuous function $\phi$ on $S_{0}$ the following is equivalent

- $\mu_{0}$ is determining for $\left(S_{0}, \phi\right)$

- The following convergence of free energies holds:

$$
\lim _{T \rightarrow 0} \lim _{N \rightarrow \infty} F_{N, \phi, T_{N}}=\inf _{\mathcal{P}\left(S_{0}\right)} E_{\phi}
$$

- The following weak convergence of the expectations $\mathbb{E}_{T, \phi}\left(\delta_{N}\right)$ of the empirical measure $\delta_{N}$ holds:

$$
\lim _{T \rightarrow 0} \lim _{N \rightarrow \infty} \mathbb{E}_{T, \phi}\left(\delta_{N}\right)=\mu_{\left(S_{0}, \phi\right)}
$$

We recall that the inverse of the infimum of the functional $E$ on $\mathcal{P}(K)$, for a given compact set $K$ in $\mathbb{R}^{d}$, is usually called the (Wiener) capacity of $K$ (see Section 6.1 for the general weighted setting).

Coming back to the energy approximation property in Section 1.1 we will also show, in Section 3.3, that the approximating sequence in question can be constructed quasiexplicitly.

\subsection{Relations to Bernstein-Markov measures, orthogonal polynomials and Ull- man's criterion}

Now specialize to the two-dimensional case and identify $\mathbb{R}^{2}$ with $\mathbb{C}$. Then the Gibbs measure (1.3) may, for $\phi=0$, be expressed as

$$
\mu_{\beta_{N}}^{(N)}:=\frac{1}{Z_{N, \beta_{N}}}\left|D^{(N)}\right|^{p_{N}} \mu_{0}^{\otimes N}, p_{N}:=2 \frac{1}{T_{N}(N-1)}
$$

where $D^{(N)}\left(z_{1}, \ldots, z_{N}\right)$ denotes the Vandermonde determinant, i.e. the polynomial on $\mathbb{C}^{N}$ defined by

$$
D^{(N)}\left(z_{1}, \ldots, z_{N_{k}}\right)=\prod_{1 \leq i<j \leq N}\left(z_{i}-z_{j}\right)=\operatorname{det}_{1 \leq i, j \leq N}\left(z_{i}^{j-1}\right)
$$

Accordingly, the corresponding partition function $Z_{N, T_{N}}$ is equal to the $L^{p_{N}}$-norm of $D^{(N)}$. More generally, introducing an exterior potential $\phi$ corresponds to replacing the $L^{p_{N}}-$ norms with weighted norms, i.e replacing $\mu_{0}$ with $e^{-T_{N}^{-1} \phi} \mu_{0}$. Note that for a fixed $p$, the corresponding $T_{N}$, tend to zero as $N \rightarrow \infty$, i.e. a fixed $p$ induces a vanishing limiting temperature $T$ (in terms of the mean field setup in the previous section). A notion of a phase transition for the Coulomb gas with respect to the parameter $p$ - from a liquid to a crystalline phase, as $p$ is increased towards a critical value - has been discussed extensively in the physics literature, supported by numerical studies [54] (and related to microscopic large deviation principles in [52, Section 4.6]). But this notion is different than the zeroth-order phase transitions discussed here, where the parameter $T$ is decreased towards zero. 
The Coulomb gas has been studied extensively in connection to Random Matrix Theory, in particular in the case when $\mu_{0}$ is Lesbegue measure on $\mathbb{R} \subset \mathbb{R}^{2}$ with $\phi$ of sufficent growth at infinity and $p=2$. The corresponding Gibbs measure $\mu_{\beta_{N}}^{(N)}$ then arises as the eigenvalue distribution of a Hermitian matrix [46, 41, 31] (see also [28, 1] for the case of general $p$ ). The asymptotics of the corresponding free energies, as $T_{N} \rightarrow 0$ was established in [38, Thm 2.1] and the LDP in [4].

We recall that a measure $\mu_{0}$ in $\mathbb{C}$ is said to satisfy a Bernstein-Markov inequality with weight $\phi$ if, for any given $\epsilon>0$ there exists a constant $C$ such that

$$
\sup _{S_{0}}\left|p_{k}\right|^{2} e^{-k \phi} \leq C e^{\epsilon k} \int_{\mathbb{C}}\left|p_{k}\right|^{2} e^{-k \phi} \mu_{0}
$$

for all polynomials $p_{k}$ on $\mathbb{C}$, where $k$ denotes the degree of $p_{k}$ (see the survey [17]). For such a measure the existence of the limit $f_{\phi}(0)$ of the corresponding $N$-particle free energies at $T=0$ was established in [15]. In fact, if the Bernstein-Markov inequality holds for all weights then the LDP holds at zero temperature, by the results in [5] (see also [16] for a different approach and [57] for relations to random polynomials). In view of Theorem 1.3 this means that the Coulomb gas on a measure $\mu_{0}$ with compact support $S_{0}$, which satisfies the weighted Bernstein-Markov inequality for $\left(S_{0}, \phi\right)$ - but which is not determining for $\left(S_{0}, \phi\right)$ - exhibits a zeroth-order phase transition at $T=0$. A general procedure for constructing such measures is explained in [17], where a concrete example of Totik on the interval is reported (see the appendix). We thus arrive at the following corollary, exhibiting a zeroth-order phase transition:

Corollary 1.4. Let $K$ be a compact domain in $\mathbb{C}$ with smooth boundary or equal to a disjoint finite union of intervals in $\mathbb{R}$. For any given continuous function $\phi$ on $K$ there exists a measure $\mu_{0}$ with support $K$ such that $\mu_{0}$ is absolutely continuous wrt $d x$ and such that the corresponding $2 d$ Coulomb gas satisfies a $L D P$ for any $T \in[0, \infty[$ with a rate functional which is discontinuous at $T=0$ in the sense of Gamma-convergence. More precisely, in the case $\phi=0$, the function

$$
f(T):=-\lim _{N \rightarrow \infty} \frac{T_{N}}{N} \log \int_{\mathbb{R}^{N}}\left|D^{(N)}\right|^{\frac{2}{T_{N} N}} \mu_{0}^{\otimes N} T=\lim _{N \rightarrow \infty} T_{N}
$$

is well-defined on $[0, \infty[$, continuous on $] 0, \infty[$, but discontinuous at $T=0$ (and similarly for a general $\phi$ ).

The property of being determining can, for a non-polar measure $\mu_{0}$, be viewed as a potential-theoretic refinement of the Bernstein-Markov inequality, where a polynomial $p_{k}$ of degree $k$ is replaced by $e^{T^{-1}} \psi_{\mu}$ for a general measure $\mu$ and positive number $T^{-1}$, playing the role of $k$. This was first shown in [12] in a general complex geometric setting. In Section 4 we will extend these notions to the case of general pair interaction potentials (the notion of Bernstein-Markov measures for Riesz interaction was introduced in [18]).

\subsubsection{Orthogonal polynomials and Ullman's criterion}

We recall that the Bernstein-Markov-inequality has it roots in the theory of orthogonal polynomials on $\mathbb{R}$. In fact, for a measure $\mu_{0}$ on $\mathbb{R}$ with compact and regular support the Bernstein-Markov inequality is equivalent to the notion of regular measures on $\mathbb{R}$ introduced in [55], whose definition involves the asymptotics of the degree $N$ orthogonal polynomials $p_{N}$ associated to $\mu_{0}$ (see the proof of Prop 4.13). In the case when the support of $\mu_{0}$ is $[-1,1]$ this notion goes back to Ullman. He also introduced the notion of determining measures on $[-1,1]$ to get a sufficient condition for regularity, known as Ullman's criterion in the general setting of measures on $\mathbb{R}$ [55]. In view of Theorem 1.2 
Ullman's criterion naturally fits into the probabilistic setting of the Coulomb gas, since it is equivalent to the continuity properties discussed above. It should be pointed out that Ullman originally used a different, but equivalent, capacity formulation of determining measures on $[-1,1]$ (see Prop 6.2 for the relation to the present setting). The definition of determining measures in the present general potential-theoretic setting on $\mathbb{R}^{d}$ mimics the definition used in the complex-geometric setting of [12], which goes back to [43].

\subsection{Towards the case of general pair interactions}

Finally, let us make some remarks about the case when the Gibbs measure (1.3) is defined by a general proper lsc function $W$. Then it essentially follows from [29, 7, 32] that the corresponding LDP holds for $T>0$ if and only if the corresponding free energy functional $F_{T}$ is a proper lsc functional (see Theorem 4.2). However, it seems challenging to find a general potential-theoretic characterization of measures $\mu_{0}$ such that the corresponding LDP also holds at $T=0$. In view of Theorem 1.2 (and its generalization to Riesz gases 4.8) the problem of characterizing measures $\mu_{0}$ such that the LDP is "well-behaved at $T=0$ " should be more accessible also in the general case. It seems likely that the answer should be given by determining measures for rather general interactions $W$, but we shall not pursue this here and only point out that in the case when $W(x, y)=W_{\alpha}(x, y)+K(x, y)$, where $W_{\alpha}$ denote the Riesz kernel and the perturbation $K(x, y)$ is continuous on $S_{0}$ the present results (concerning the case $K=0)$ directly generalize. Indeed, since $K(x, y)$ is continuous it influences neither the energy approximation property, nor the determining property appearing in Theorem 1.1. For example, this situation naturally appears in electrostatics in $\mathbb{R}^{3}$ with the screened Coulomb interaction (Yukawa potential) $W(x, y)=e^{-m|x-y|} /|x-y|$ for a given positive number $m$.

\subsection{Further relations to previous results}

The idea of studying the Gibbs measures corresponding to a general lsc pair interaction potential $W$, in the case $T=0$, by letting $T \rightarrow 0$ goes back to [41], which builds on the variational approach introduced in [47] (where the case of a continuous $W$ was considered). In the main result of [41] it is claimed that, in general, any limit point $\mu$ in $\mathcal{P}\left(\mathbb{R}^{d}\right)$ of the empirical measure $\delta_{N}$ minimizes the corresponding energy functional $E$. However, in the case of the Coulomb gas this is contradicted by the example in Theorem 4.12, where $\mu_{0}$ has support $[-1,1]$ and is absolutely continuous wrt $d x$ (see also Example 4.4 for a simple counter-example in the weighted setting). The mistake in [41] appears to be the claimed inequality $[41,3.12]$, which, in general, requires assumptions on $\mu_{0}$.

The "only if" direction in Theorem 1.3 was first shown in the complex-geometric setting of compact Kähler manifolds $X$ in [9] and generalized to the non-compact setting pluripotential setting of $\mathbb{C}^{n}$ in [9], using a compactification argument. See also [35] for a far-reaching generalization of [8, Theorem 2.1] to general measures not charging pluripolar subsets and bounded weights.

It should also be pointed out that there are particular situations where the rate of the convergence of $\mu_{T, \phi}$ towards the equilibrium measure $\mu_{\left(S_{0}, \phi\right)}$ can be quantified. In the setting of a compact Kähler manifold $X$, studied in [8], the rate of convergence of the $L^{\infty}$ norms of the corresponding potentials $\psi_{\mu_{T, \phi}}$ was shown to be of the order $O(T) \log T^{-1}$. In particular, the case when $X$ is the Riemann sphere implies (by a compactification argument, as in [9]) that such a rate holds for the Coulomb gas in $\mathbb{R}^{2}$ with $\mu_{0}=d x$ if $\phi$ has strictly super logarithmic growth. More general and precise quantitative convergence results for Coulomb gases in any dimension have very recently been established in 
[2] (see also [1, formula 18] for an explicit integral formula for $\mu_{T, \phi}$ in the case of the Coulomb gas on $\mathbb{R}$ with $\phi(x)=x^{2}$ and $\mu_{0}$ the Gaussian measure, so that $\mu_{T, \phi}$ converges, as $T \rightarrow 0$, to Wigner's semi-circle law). However, it should be stressed that in the general setting of determining measures $\mu_{0}$, studied in the present paper, one can not hope for quantitative rates, unless regularity assumptions on $\mu_{0}$ are imposed.

\subsection{On the proofs}

The core analytic result is Theorem 1.3 and its general form 3.10 (which applies to any Riesz interaction with $\alpha \leq 2$ ), saying, in particular, that the measure $\mu_{0}$ is determining iff the corresponding free energy $f(T)$ is continuous as $T \rightarrow 0$. The proof of the "if" direction" mimics the variational proof of a similar result in the complex geometric setting on a compact Kähler manifold $X$ [8, Theorem 2.1] (which applies, in particular, to the case $d=\alpha=2$ by taking $X$ to be the Riemann sphere). An important ingredient is the potential-theoretic analog of the Bernstein-Markov property for determining measures in Prop 3.6 (proved in [12] in the complex geometric setting). In the present setting we also have to deal with the non-compactness of $\mathbb{R}^{d}$ and the non-local properties of the fractional Laplacian $-(-\Delta)^{\alpha / 2}$ (for $\alpha<2$ ). In the case when $d=\alpha=2$ the "only if" direction could alternatively be deduced from the generalization in [35] of [8, Theorem $2.1]$ to arbitrary measures $\mu_{0}$, not charging pluripolar subsets. However, the proof in [35] (which is not variational) appears to exploit some special local features of the complex geometric setting, which do not seem to apply when $\alpha \neq 2$. Here we instead use a variational approach, which has the virtue of only demanding some rather general axioms of potential theory (compare Remark 2.1).

\subsection{Acknowledgments}

It is a pleasure to thank Sébastien Boucksom, David Witt-Nyström, Vincent Guedj and Ahmed Zeriahi for the stimulating collaborations [11, 12, 13], which provided important motivation for the present paper and Norm Levenberg for helpful comments. Thanks also to the referees for very helpful comments that improved the exposition. This work was supported by grants from the ERC, the KAW foundation and the Göran Gustafsson foundation.

\subsection{Organization}

In Section 2 we introduce the weighted potential theory needed for the proofs of the main analytic results. In particular, a dual representation of the energy $E(\mu)$ as a Legendre transform is given. In Section 1.1 we reformulate the Energy-Approximation Property in terms of Gamma-convergence of the free energy functional $F_{T}$, which in turn is a given a dual formulation using Legendre transforms. Then in Section 3.2 the proofs of the main analytic results for Riesz interactions are given, by relating Gamma-convergence of $F_{T}$ to determining measures. The connections to large deviation principles is studied in Section 4 and connections to Bernstein-Markov inequalities are explored. The results are then reformulated in terms of phase transitions in Section 5. In the appendix some construction of measures $\mu_{0}$ are provided, which illustrate the sharpness of the main results.

\subsection{General notation}

We will denote by $\mathcal{P}_{c}\left(\mathbb{R}^{d}\right)$ the space of all compactly supported probability measures on $\mathbb{R}^{d}$ and by $\mathcal{P}(K)$ the subset consisting of measures supported on a compact subset $K$ of $\mathbb{R}^{d}$. We endow the space $\mathcal{P}(K)$ with the weak topology. Throughout the paper we fix a probability measure $\mu_{0}$ with compact support, denoted by $S_{0}$. Given a compact subset $K$ 
of $\mathbb{R}^{d}$ we will denote by $\mathcal{C}(K)$ the space of all continuous functions on $K$.

We recall that a function $f$ on a topological space $X$, taking values in $] \infty, \infty]$ is lower semi-continuous (lsc) if $\{f \leq \alpha\}$ is closed for any $\alpha \in] \infty, \infty]$. We will say that $f$ is proper lower-semicontinuous under the further assumption that $f$ is not identically equal to $\infty$ (following standard terminology in convex analysis). If $X$ is compact and $f$ is lsc the latter condition equivalently means that $\inf _{X} f$ is finite. Hence, under the map $f \mapsto f-\inf _{X} f$ the space of proper lsc functions on $X$ corresponds to the space of rate functionals, in the sense of large deviation theory. Finally, it will be convenient to work with inverse temperatures $\beta_{N}:=T_{N}^{-1}$ and $\beta:=T^{-1}$ rather than temperatures.

\section{Weighted potential theory and Legendre transforms}

In this section we develop the weighted potential theory needed for the proofs of the main results. The key result is the Legendre transform representation of the energy in Theorem 2.12. The presentation is inspired by the complex-geometric framework in $[11,12,13]$, which covers in particular the Coulomb case in $\mathbb{R}^{2}$ (see also [51, 26] for different points of view).

\subsection{Potential-theoretic preliminaries}

We start by recalling some basic potential-theoretic results. We follow the classical reference [42], but with a different sign convention for the kernels and the potentials (ensuring that the potentials are subharmonic in the Coulomb case).

We will denote by $W_{\alpha}(x, y)$ the Riesz kernel with parameter $\left.\alpha \in\right] 0, d[$, i.e. the lsc function on $\mathbb{R}^{d} \times \mathbb{R}^{d}$ defined by

$$
W_{\alpha}(x, y):=\frac{1}{|x-y|^{d-\alpha}} .
$$

When $d=2$ we will allow the case $\alpha=d=2$, by setting

$$
W_{2}(x, y)=-2 \log |x-y|
$$

The definition ensures that when $d \geq 2$ the function $W_{2}(x, y)$ is a (up to multiplication by a negative constant) a Green's kernel for the Laplacian $\Delta$ on $\mathbb{R}^{d}$. Accordingly, we will refer to the case $\alpha=2$ as the "Coulomb case" and the special case $\alpha=2=d$ the "logarithmic case".

The energy $E(\mu)$ of a measure $\mu \in \mathcal{P}_{c}\left(\mathbb{R}^{d}\right)$ is defined by

$$
\left.\left.E(\mu)=\frac{1}{2} \int_{\mathbb{R}^{d}} W_{\alpha} \mu \otimes \mu \in\right]-\infty, \infty\right]
$$

Given a measure $\mu$ on $\mathbb{R}^{d}$ we will denote by $\psi_{\mu}$ its potential:

$$
\psi_{\mu}(x):=-\int_{\mathbb{R}^{d}} W_{\alpha}(x, y) \mu(y),
$$

Since $W_{\alpha}$ is symmetric the following symmetry property holds:

$$
\int \psi_{\nu} \mu=\int \nu \psi_{\mu}
$$

if $\mu$ and $\nu$ are in $\mathcal{P}_{c}\left(\mathbb{R}^{d}\right)$ and of finite energy. Moreover, $W_{\alpha}$ defines a strictly positive definite bilinear form in the following sense:

$$
-\int\left(\psi_{\mu}-\psi_{\nu}\right)(\mu-\nu) \geq 0
$$


with equality iff $\nu=\mu$. This implies that the map $\mu \mapsto \psi_{\mu}$ is injective and we will denote the inverse operator by $\Delta_{\alpha}$, which coincides with the ordinary Laplacian when $\alpha=2 .{ }^{1}$ A bounded set $S$ is said to be polar if $S \subset\left\{\psi_{\mu}=-\infty\right\}$ for some measure $\mu$ (equivalently, $S$ has vanishing outer capacity) ${ }^{2}$. If $S$ is compact then $S$ is polar iff $E(\mu)=\infty$ for any measure $\mu \in \mathcal{P}(S)$. A property is said to hold quasi-everywhere (q.e) if it holds on the complement of a polar set.

If $\mu_{j} \in \mathcal{P}(K)$ for a compact subset $K$ and $\mu_{j} \rightarrow \mu$ weakly, then, for any given $x \in \mathbb{R}^{d}$

$$
\limsup _{j \rightarrow \infty} \psi_{\mu_{j}}(x) \leq \psi_{\mu}(x)
$$

as follows directly from the lower semi-continuity of $W$. Moreover, for q.e. $x$ in $\mathbb{R}^{d}$,

$$
\limsup _{j \rightarrow \infty} \psi_{\mu_{j}}(x)=\psi_{\mu}(x)
$$

(see [42, Therem 3.8, page 190]). As a consequence [42, page 191, Remark 2],

$$
\left(\limsup _{j \rightarrow \infty} \psi_{\mu_{j}}\right)^{*}=\psi_{\mu}
$$

on all of $\mathbb{R}^{d}$, where the limsup is defined point-wise and $f^{*}$ denotes the upper semicontinuous regularization of a function $f$ on $\mathbb{R}^{d}$ :

$$
f^{*}(x)=\sup \left\{f\left(x_{j}\right): x_{j} \rightarrow x\right\}
$$

where the sup runs over all sequences $x_{j}$ converging to $x$. We will be mainly interested in the case when $\alpha \leq 2$, since the following domination principle then applies [42, Thm 1.29]: for a given constant $C$

$$
\psi_{\nu} \leq \psi_{\mu}+C \mu-\text { a.e } \Longrightarrow \psi_{\nu} \leq \psi_{\mu}+C,
$$

assuming that $\mu$ has finite energy.

Remark 2.1. When $\alpha \leq 2$ it is also known that the space of potentials is preserved under the max operation [42, Thm 1.31] (the case $\alpha=2$ follows directly from subharmonicity). But for our purposes it will be enough to use the domination principle. This should be useful in order to extend Theorem 3.11 to more general kernels $W(x, y)$ appearing in axiomatic potential theory, where the domination principle (aka the second maximum principle) is often is taken as an axiom [42, Page 364]. For example, the domination principle holds when $W(x, y)$ is the potential kernel of a Markov process satisfying Hunt's hypothesis (H) [30] (then $-\psi_{\mu}$ is called the excessive function associated to $\mu$ ). But we shall not go further into this here.

In order to simplify the notation we we will omit the dependence on $\alpha$ of the potentialtheoretic objects associated to the Riesz kernel $W_{\alpha}(x, y)$, such as the energy $E(\mu)$, potentials $\psi_{\mu}$ and the corresponding inverse operator $\Delta$ (which coincides with the Laplacian when $\alpha=2$ ). As $\alpha$ will be fixed this should not cause any confusion.

\footnotetext{
${ }^{1}$ In general, $\Delta_{\alpha}$ is a fractional Laplacian $\Delta_{\alpha}:=-(-\Delta)^{\frac{\alpha}{2}}$ in the sense of functional calculus, which in the case $\alpha \in] 0,2]$ corresponds precisely to the generator of a symmetric stable Levy process [19, 20].

${ }^{2}$ This terminology is standard, but different from the one in [42], where the terminology polar is used for the sets which are precisely equal to some $\left\{\psi_{\mu}=-\infty\right\}$ (such sets are called completely polar in modern terminology).
} 
Priors leading to well-behaved Coulomb, Riesz gases vs zeroth-order phase transitions

\subsection{Function spaces}

We will use the notation

$$
\mathcal{L}_{c}\left(\mathbb{R}^{d}\right):=\left\{\psi: \psi=\psi_{\mu}+C, \mu \in \mathcal{P}_{c}\left(\mathbb{R}^{d}\right), C \in \mathbb{R}\right\}
$$

We endow the space $\mathcal{L}_{c}\left(\mathbb{R}^{d}\right)$ with the $L_{\text {loc }}^{1}$-topology induced from the inclusion $\mathcal{L}_{c}\left(\mathbb{R}^{d}\right) \Subset$ $L_{\text {loc }}^{1}\left(\mathbb{R}^{d}\right)$. Denote by $\mathcal{E}_{c}\left(\mathbb{R}^{d}\right)$ the subspace of $\mathcal{L}_{c}\left(\mathbb{R}^{d}\right)$ satisfying $E(\Delta \psi)<\infty$. Given a compact subset $K$ of $\mathbb{R}^{d}$ we will write $\mathcal{L}_{K}\left(\mathbb{R}^{d}\right)$ and $\mathcal{E}_{K}\left(\mathbb{R}^{d}\right)$ for the subspaces of $\mathcal{L}_{c}\left(\mathbb{R}^{d}\right)$ and $\mathcal{E}_{c}\left(\mathbb{R}^{d}\right)$, respectively, obtained by demanding that $\Delta \psi$ be a probability measure supported in $K$. The definitions are made so that, for any compact $S$, we have a bijection (whose inverse is $\Delta$ ) :

$$
\mu \mapsto \psi_{\mu} \mathcal{P}(S) \longleftrightarrow \mathcal{L}_{S}\left(\mathbb{R}^{d}\right) / \mathbb{R}
$$

Proposition 2.2. (Compactness) Let $S$ be a compact subset of $\mathbb{R}^{d}$ and fix a closed ball $B$ containing $S$. Then the subspace of $\mathcal{L}_{S}\left(\mathbb{R}^{d}\right)$ consisting of all $\psi$ which are "mean normalized", i.e.

$$
\int_{B} \psi d x=0
$$

is compact wrt the $L_{\text {loc }}^{1}$-topology. Moreover, given a compact subset $K$ there exists a constant $C$ such that

$$
\sup _{K} \psi \leq \frac{\int_{B} \psi d x}{\int_{B} d x}+C
$$

on $\mathcal{L}_{S}\left(\mathbb{R}^{d}\right)$.

\section{Proof. Step 1: Compactness for "mean-normalized functions"}

The compactness is a consequence of the following general properties of the kernel $-W_{\alpha}$ : it is symmetric, usc, continuous on the complement of the diagonal, in $L_{l o c}^{1}$ and the corresponding integral operator yields a bijection, as in formula (2.7). To see this take any $\mu \in \mathcal{P}(S)$ and first observe that

$$
\int_{B} \psi_{\mu} d x=\int f \mu
$$

for the continuous function $f:=\psi_{\nu}$ with $\nu:=1_{B} d x / \int_{B} d x$ (using the symmetry property (2.2)). Now decompose any mean-normalized $\psi$ as

$$
\psi=\left(\psi+\int f \mu\right)-\int f \mu
$$

Since $\psi$ is normalized the bijection (2.7) shows that the first term equals $\psi_{\mu}$, where $\mu:=\Delta \psi$. Let now $\psi_{j}$ be a sequence in $\mathcal{L}_{S}\left(\mathbb{R}^{d}\right)$ and set $\mu_{j}:=\Delta \psi_{j}$. Since $S$ is compact the space $\mathcal{P}(S)$ is also compact and hence there exists $\mu \in \mathcal{P}(S)$ such that $\mu_{j} \rightarrow \mu$ in $\mathcal{P}(S)$, after perhaps passing to a subsequence. All that remains is thus to verify that $\psi_{\mu_{j}} \rightarrow \psi_{\mu}$ in $L_{l o c}^{1}$. To this end first note that, since $-W_{\alpha}$ is usc, we have

$$
\limsup _{j \rightarrow \infty} \psi_{\mu_{j}} \leq \psi
$$

Moreover, by symmetry,

$$
\int_{B} \psi_{\mu_{j}} d x=\int f \mu_{j} \rightarrow \int f \mu:=\int_{B} \psi d x
$$

since $f$ is continuous. But then it follows from general integration theory that $\psi_{\mu_{j}} \rightarrow \psi$ in $L^{1}(B, d x)$. Finally, the $L_{l o c}^{1}$ convergence on the complement of $S$ follows directly from the fact that $W_{\alpha}$ is continuous on the complement of the diagonal. 
Priors leading to well-behaved Coulomb, Riesz gases vs zeroth-order phase transitions

Step 2: the upper bound on $\sup _{K} \psi$

By the previous step it will be enough to prove that the functional $\psi \mapsto \sup _{K} \psi$ is usc on $\mathcal{L}_{S}\left(\mathbb{R}^{d}\right)$. To this end first observe that, if $\mu_{j} \rightarrow \mu$ in $\mathcal{P}(S)$ and $x_{j} \rightarrow x$ then

$$
\limsup \psi_{\mu_{j}}\left(x_{j}\right) \leq \psi_{\mu}(x),
$$

using that the kernel $-W_{\alpha}$ is usc (see [42, Thm 1.3]). As consequence, if $\psi_{j} \rightarrow \psi_{\infty}$ in $\mathcal{L}_{S}\left(\mathbb{R}^{d}\right)$ then the previous inequality still holds if $\psi_{\mu_{j}}$ and $\psi_{\mu}$ are replaced by $\psi_{j}$ and $\psi_{\infty}$, respectively (using the decomposition argument in the previous step). Now, taking $x_{j}$ so that $\psi_{j}\left(x_{j}\right)=\sup _{K} \psi_{j}$ concludes the proof of Step 2 .

\subsection{Energy functionals on $\mathcal{P}(S)$ and the equilibrium measure of a weighted set} $(S, \phi)$

If $\mu$ has compact support and $\phi$ is a continuous function on $\mathbb{R}^{d}$ we set

$$
\left.\left.E_{\phi}(\mu):=-\frac{1}{2} \int \psi_{\mu} \mu+\int \phi \mu \in\right]-\infty, \infty\right]
$$

The definition is made so that $E_{0}(\mu):=E(\mu)$ is the classical energy of $\mu$ (formula (2.1)).

Proposition 2.3. Let $S$ be a non-polar subset. Then the restriction of $E$ to $\mathcal{P}(S)$ is lsc and strictly convex. Hence, so is $E_{\phi}$ for any given continuous function $\phi$.

Proof. This follows from (and is equivalent to) the positivity (2.3).

Thus, if $S$ is a non-polar compact subset, then $E_{\phi}$ admits a unique minimizer $\mu_{(S, \phi)}$ on $\mathcal{P}(S)$, which is called the equilibrium measure of the weighted set $(S, \phi)$.

Given a non-polar compact weighted set $(S, \phi)$ it will also be convenient to consider a normalized version of the functional $E_{\phi}$ on $\mathcal{P}(S)$ defined by

$$
E_{\omega_{\phi}}:=E_{\phi}-\inf _{\mathcal{P}(S)} E_{\phi},
$$

which is invariant under $\phi \mapsto \phi+C$ for any constant $C$.

Remark 2.4. The notation is inspired by the corresponding complex-geometric setup, using the notation in [5,9], applied to the special case when $d=\alpha=2$. In this case, when $\phi$ is expressed as the restriction to $S$ of a function with logarithmic growth in $\mathbb{C}$, the symbol $\omega_{\phi}$ denotes the signed measure $\omega_{\phi}:=\Delta \phi$ (extended by zero from $\mathbb{C}$ to the Riemann sphere) and $E_{\omega_{\phi}}$ is the corresponding pluricomplex energy. Since the normalized energy, defined by formula (2.10), is invariant under $\phi \mapsto \phi+C$ it depends, indeed, only on $\Delta \phi$.

Lemma 2.5. Given a weighted non-polar compact subset $(S, \phi)$ the potential $\psi_{\mu} \in \mathcal{L}_{c}\left(\mathbb{R}^{d}\right)$ of the equilibrium measure $\mu:=\mu_{(S, \phi)}$ has the following property: there exists a constant $C$ such that

$$
\begin{gathered}
\text { (i) } \psi_{\mu} \leq \phi+C \text { q.e.on } S \\
\text { (ii) } \psi_{\mu} \geq \phi+C \text { on } S_{\mu}
\end{gathered}
$$

where $S_{\mu}$ denotes the support of $\mu$.

Proof. This goes back to Frostman and is proved in [26, Theorem 1.2] when $S=\mathbb{R}^{n}$ and in [51] in the logarithmic case. The proof in the general case is essentially the same and follows from rather general variational considerations. Indeed, one first observes that $u_{\mu}:=-\left(\psi_{\mu}-\phi\right)$ is a sub-gradient for the functional $E_{\phi}(\mu)$. Hence, if $\mu$ minimizes $E_{\phi}$ on $\mathcal{P}(S)$, then $\left\langle u_{\mu}, \nu-\mu\right\rangle \geq 0$ for any $\nu \in \mathcal{P}(S)$ of finite energy. Applying this inequality to 
$\nu=\mu(1+f)$ for any $f \in L^{\infty}(\mu)$ such that $\int f \mu=0$ and $\|f\|_{L^{\infty}(\mu)}<1$ gives $\left\langle u_{\mu}, f \mu\right\rangle \geq 0$. Replacing $f$ with $-f$ yields the reversed inequality, showing that $\left\langle u_{\mu}, f \mu\right\rangle=0$ for any $f$ such that $\int f \mu=0$. But this implies that $u_{\mu}=-C \mu$-a.e. for some constant $C$. Since, $u$ is lsc it follows that $u_{\mu} \leq-C$ on $S_{\mu}$. As a consequence, $\left\langle u_{\mu}, \nu\right\rangle \geq-C$ for any $\nu \in \mathcal{P}(S)$ of finite energy, which implies $u_{\mu} \geq-C$ q.e. on $S$ (using that $u_{\mu}$ is lsc and that any subset of positive capacity has a compact subset of positive capacity).

To a compact weighted set $(S, \phi)$ we now attach the following function in $\mathcal{L}_{S}\left(\mathbb{R}^{d}\right)$ :

$$
\psi_{(S, \phi)}:=\psi_{\mu_{(S, \phi)}}-C
$$

where $C$ is the constant appearing in the previous lemma.

\subsection{The projection operator $P_{S}$}

In this section we assume that $\alpha \leq 2$ (so that the domination principle (2.6) applies).

Now assume given a weighted set $(S, \phi)$, i.e. a subset $S$ of $\mathbb{R}^{d}$ and a continuous function $\phi \in C(S)$. Consider the function

$$
\left(\Pi_{S} \phi\right)(x):=\sup _{\mathcal{L}_{c}\left(\mathbb{R}^{d}\right)}\{\psi(x): \quad \psi \leq \phi \text { on } S\}
$$

Its upper semi-continuous regularization will be denoted by

$$
P_{S} \phi:=\left(\Pi_{S} \phi\right)^{*}
$$

Proposition 2.6. Let $(S, \phi)$ be a weighted subset and assume that $S$ is compact and non-polar. Then

$$
\psi_{(S, \phi)}=P_{S} \phi
$$

In particular, $\Delta\left(P_{S} \phi\right)$ is the unique minimizer of the functional $E_{\phi}$ on $\mathcal{P}(S)$. Moreover, as a consequence of (2.13),

$$
P_{S} \phi \in \mathcal{E}_{S}\left(\mathbb{R}^{d}\right)
$$

and the operator $P_{S}$ satisfies the following "orthogonality relation"

$$
\int\left(\phi-P_{S} \phi\right) \Delta\left(P_{S} \phi\right)=0 .
$$

More generally, if $S$ is bounded and equal to the union of increasing compact subsets, then $P_{S} \phi \in \mathcal{E}_{\bar{S}}\left(\mathbb{R}^{d}\right)$ for any given continuous function $\phi$ on $\mathbb{R}^{d}$.

Proof. Combining Lemma 2.5 with the domination principle (2.6) gives $\Pi_{S} \phi \leq \psi_{(S, \phi)}$ and hence $P_{S} \phi \leq \psi_{(S, \phi)}$. Indeed, let $\psi$ be a candidate for the sup defining $\Pi_{S} \phi$. By Lemma (2.19) $\psi \leq \phi \leq \psi_{(S, \phi)}$ on the support of $\Delta\left(\psi_{(S, \phi)}\right)$. Hence, the domination principle implies that $\psi \leq \psi_{(S, \phi)}$ everywhere. Moreover, since, by Lemma 2.5, $\psi_{(S, \phi)} \leq \phi$ on $S-N$ where $N$ is polar we also have $\psi_{(S, \phi)} \leq P_{S-N} \phi$. The proof is thus concluded by invoking the fact that $P_{T \cup N} \phi=P_{T} \phi$ for any bounded Borel set $T$ and polar subset $N$ (applied to $T:=S-N$ ). To see this first note that, trivially, $P_{T \cup N} \leq P_{T}$. To prove the converse fix $\psi_{N} \in \mathcal{L}_{c}\left(\mathbb{R}^{d}\right)$ such that $\psi_{N}=-\infty$ on $N$ and $\psi_{N} \leq \phi$ on $T$. The existence of $\psi_{N}$ follows form the fact that, by definition, any polar subset $N$ is contained in the $-\infty$-locus of some potential $\psi_{N}$. Using the compactness of $S$ we can then arrange that $\psi_{N} \in \mathcal{L}_{c}\left(\mathbb{R}^{d}\right)$ and $\psi_{N} \leq \phi$ on $S$ and hence also $\psi_{N} \leq \phi$ on $T$. Thus, for any $\psi \in \mathcal{L}_{c}\left(\mathbb{R}^{d}\right)$ such that $\psi \leq 0$ on $T$ we get $\psi_{\epsilon}:=(1-\epsilon) \psi+\epsilon \psi_{N} \leq \phi$ on $T \cup N$. Hence, $\psi_{\epsilon} \leq \Pi_{T \cup N} \phi$. Letting $\epsilon \rightarrow 0$ gives $\Pi_{T} \phi \leq \Pi_{T \cup N} \phi$ on the complement of the polar subset $\left\{\psi_{N}=-\infty\right\}$ and hence $P_{T} \phi \leq P_{T \cup N} \phi$ everywhere, as desired (using that if $\psi_{\mu} \leq \psi_{\nu}+C$ q.e. for a 
measure $\nu$ of finite energy and a constant $C$, then $\psi_{\mu} \leq \psi_{\nu}+C$ everywhere, as a special case of the domination principle, since $\nu$ does not charge polar subsets).

Finally, if $S$ it the union of increasing compact subsets $K_{i}$ then $P_{S} \phi \leq \psi_{i}:=P_{K_{i}} \phi$ for any $i$. By the previous step $\psi_{i}$ is a decreasing sequence in $\mathcal{E}_{\bar{S}}\left(\mathbb{R}^{d}\right)$ and $\psi_{i} \leq \phi$ on $K_{i}-N_{i}$, where $N_{i}$ is polar. Hence, by Prop 2.2, $\psi_{i}$ converges in $L_{l o c}^{1}$ to $\psi_{\infty} \in \mathcal{E}_{\bar{S}}\left(\mathbb{R}^{d}\right)$, where $\psi_{\infty} \leq \phi$ on $S-N$, where $N$ is the union of the polar sets $N_{i}$ and hence polar (since the outer capacity is sub-additive). This means that $P_{S} \phi \leq \psi_{\infty}$ and $\psi_{\infty} \leq P_{S-N} \phi$. Since, as explained in the proof of the previous step, $P_{S-N} \phi=P_{S} \phi$ this concludes the proof.

Lemma 2.7. Let $S$ be a compact subset. The operator $P_{S}$ defines a (non-linear) increasing concave operator from $\mathcal{C}(S)$ onto $\mathcal{L}_{S}\left(\mathbb{R}^{d}\right)$. Moreover, for any $\psi \in \mathcal{L}_{c}\left(\mathbb{R}^{d}\right)$

$$
P_{S} \psi \geq \psi
$$

with equality if $\psi \in \mathcal{L}_{S}\left(\mathbb{R}^{d}\right)$. Hence, $P_{S}$ is a projection operator, i.e. $P_{S}^{2}=P_{S}$.

Proof. It follows directly from the definitions that $\phi_{0} \leq \phi_{1}$ implies that $\Pi_{S} \phi_{0} \leq \Pi_{S} \phi_{1}$ and hence also $P_{S} \phi_{0} \leq P_{S} \phi_{1}$, i.e. $P_{S}$ is increasing. Concavity of $\Pi_{S}$ follows directly from the definition as a sup of linear functionals (defined by evaluation) and this implies the concavity of $P_{S}$, as well. Next, if $\psi \in \mathcal{L}_{c}\left(\mathbb{R}^{d}\right)$ then $\Pi_{S} \psi \geq \psi$ (since $\psi$ is a candidate for the sup defining $\left.\Pi_{S} \psi\right)$. Since $\psi^{*}=\psi$ it follows that $P_{S} \psi \geq \psi$. The fact that $P_{S} \psi \in \mathcal{L}_{S}\left(\mathbb{R}^{d}\right)$ was proved in the previous proposition. Conversely, if $\psi \in \mathcal{L}_{S}\left(\mathbb{R}^{d}\right)$ then it follows, directly from the domination principle, that $\Pi_{S} \psi \leq \psi$ and hence also $P_{S} \psi \leq \psi \leq P_{S} \psi$, which proves the projection property in question.

\subsection{The primitive functional $\mathcal{E}$ on $\mathcal{L}_{c}\left(\mathbb{R}^{d}\right)$ and its projection $\mathcal{F}$ to $C(S)$.}

The operator $\Delta$ can be naturally identified with a one-form on the convex space $\mathcal{L}_{c}\left(\mathbb{R}^{d}\right) \cap C\left(\mathbb{R}^{d}\right)$ :

$$
\langle\Delta \psi, v\rangle:=\int \Delta \psi v
$$

According to the next proposition this one-form admits a primitive that we shall denote by $\mathcal{E}$, i.e. a functional on $\mathcal{L}_{c}\left(\mathbb{R}^{d}\right) \cap C\left(\mathbb{R}^{d}\right)$ whose differential is the operator $\Delta$ :

$$
(d \mathcal{E})(\psi)=\Delta \psi
$$

Since $\mathcal{L}_{c}\left(\mathbb{R}^{d}\right) \cap C\left(\mathbb{R}^{d}\right)$ is convex the primitive $\mathcal{E}$ is uniquely determined up to an overall constant, which may be fixed by imposing the normalization condition

$$
\mathcal{E}\left(\psi_{0}\right)=0
$$

for a fixed reference element $\psi_{0} \in \mathcal{L}_{c}\left(\mathbb{R}^{d}\right) \cap C\left(\mathbb{R}^{d}\right)$. We will sometimes use a subscript $\mathcal{E}_{\psi_{0}}$ to indicate the dependence on the choice of $\psi_{0}$. Integrating along an affine line in $\mathcal{L}_{c}\left(\mathbb{R}^{d}\right) \cap C\left(\mathbb{R}^{d}\right)$ suggests the following explicit formula, that we shall take as the definition of $\mathcal{E}_{\psi_{0}}$ on the whole space $\mathcal{L}_{c}\left(\mathbb{R}^{d}\right)$ :

$$
\mathcal{E}_{\psi_{0}}(\psi):=\frac{1}{2} \int\left(\psi-\psi_{0}\right)\left(\Delta \psi+\Delta \psi_{0}\right) \in[-\infty, \infty[
$$

Moreover, it will be convenient to allow the reference $\psi_{0}$ to be in $\mathcal{E}_{c}\left(\mathbb{R}^{d}\right)$.

Proposition 2.8. The functional $\mathcal{E}_{\psi_{0}}$ on $\mathcal{L}_{c}\left(\mathbb{R}^{d}\right)$ has the following properties

(1) $\mathcal{E}_{\psi_{0}}(\psi)>-\infty$ iff $\psi \in \mathcal{E}_{c}\left(\mathbb{R}^{d}\right)$. Moreover, $\Delta \psi_{j} \rightarrow \Delta \psi$ in $\mathcal{P}(S)$ and $E\left(\Delta \psi_{j}\right) \rightarrow E\left(\Delta \psi_{j}\right)$ for $S$ compact iff $\psi_{j} \rightarrow \psi$ in $L_{l o c}^{1}$ and $\mathcal{E}_{\psi_{0}}\left(\psi_{j}\right) \rightarrow \mathcal{E}_{\psi_{0}}(\psi)$

(2) $\mathcal{E}_{\psi_{0}}$ is usc on $\mathcal{L}_{c}\left(\mathbb{R}^{d}\right)$ 
Priors leading to well-behaved Coulomb, Riesz gases vs zeroth-order phase transitions

(3) Given $\psi_{1}, \psi_{2} \in \mathcal{E}_{c}\left(\mathbb{R}^{d}\right)$ we have

$$
{\frac{d \mathcal{E}_{\psi_{0}}\left(\psi_{1}+t\left(\psi_{2}-\psi_{1}\right)\right)}{d t}}_{\mid t=0}=\int \Delta \psi_{1}\left(\psi_{2}-\psi_{1}\right)
$$

(4) $\mathcal{E}_{\psi_{0}}$ is concave on $\mathcal{L}_{c}\left(\mathbb{R}^{d}\right)$

(5) $\mathcal{E}_{\psi_{0}}$ is strictly increasing on $\mathcal{E}_{c}\left(\mathbb{R}^{d}\right)$ : if $\psi \leq \Psi$, then $\mathcal{E}_{\psi_{0}}(\psi) \leq \mathcal{E}_{\psi_{0}}(\Psi)$ with equality iff $\psi=\Psi$.

(6) The following cocycle property holds: for any triple $\psi_{i} \in \mathcal{E}_{c}\left(\mathbb{R}^{d}\right)$ the difference

$$
\mathcal{E}_{\psi_{0}}\left(\psi_{2}\right)-\mathcal{E}_{\psi_{0}}\left(\psi_{1}\right)
$$

is independent of $\psi_{0}$.

(7) For any $c \in \mathbb{R}$ we have $\mathcal{E}_{\psi_{0}}(\psi+c)=\mathcal{E}_{\psi_{0}}(\psi)+c$

Proof. To prove item 1 we may, by the cocycle property 6 proved below, assume that $\Delta \psi_{0}=\rho d x$ for a continuous function $\rho$ with compact support. Now decompose

$$
\mathcal{E}_{\psi_{0}}(\psi)-\frac{1}{2} \int \psi \Delta \psi=-\frac{1}{2} \int \psi_{0} \Delta \psi+\frac{1}{2} \int \psi \Delta \psi_{0}+C_{0}
$$

Since $\psi_{0}$ is bounded on the support of $\Delta \psi$ the first term in the rhs above is finite and so is the second one since $\psi \in L_{l o c}^{1}$. The same argument proves the convergence statement. Item 2 also follows from the previous decomposition, using that $W$ is usc, just as in the proof of Prop 2.2. As for the formula in item 3 it follows directly from the symmetry (2.2). Similarly, the concavity of $\mathcal{E}_{\psi_{0}}$ follows from the fact that $\langle\Delta u, u\rangle \geq 0$ if $u=\psi_{2}-\psi_{1}$ for $\psi_{i} \in \mathcal{E}_{c}\left(\mathbb{R}^{d}\right)$ (by the positivity (2.3)). That $\mathcal{E}_{\psi_{0}}$ is increasing follows directly from item 3, since $\Delta \psi \geq 0$ for any $\psi \in \mathcal{L}_{c}\left(\mathbb{R}^{d}\right)$ and strictly increasing follows from the domination principle when $\alpha \leq 2$. In the general case it follows from the strict concavity of $\mathcal{E}_{\psi_{0}}$, which in turn follows from the strict positivity in (2.3)). The cocycle property in item 6 follows directly from expressing $\mathcal{E}_{\psi_{0}}\left(\psi_{2}\right)-\mathcal{E}_{\psi_{0}}\left(\psi_{1}\right)$ as an integral on $[0,1]$ of the derivative $d \mathcal{E}_{\psi_{0}}\left(\psi_{1}+t\left(\psi_{2}-\psi_{1}\right)\right) / d t$ and noting that, by item 3 , the derivative is independent of $\psi_{0}$. Finally, item 7 also follows directly from item 3.

Remark 2.9. If $\psi_{0}$ is normalized so that $\psi_{0}=\psi_{\Delta \psi_{0}}$, then it follows directly from the symmetry (2.2) that $-\mathcal{E}_{\psi_{0}}\left(\psi_{\mu}\right)=E(\mu)+C_{0}$. However, it will be important to consider the functional $\mathcal{E}$ defined on all of $\mathcal{E}_{c}\left(\mathbb{R}^{d}\right)$.

Next, assume that $\alpha \leq 2$ (so that the domination principle (2.6) applies). Given a weighted compact and non-polar set $(S, \phi)$, consider the following functional defined on $C(S)$ :

$$
-\mathcal{F}_{(S, \phi)}(u):=\mathcal{E}_{\psi_{0}} \circ P_{S}(\phi-u)-\mathcal{E}_{\psi_{0}}\left(P_{S}(\phi)\right)
$$

Equivalently, this means, by the cocycle property in Prop 2.8, that, if choose the particular canonical reference weight

$$
\psi_{0}:=P_{S}(\phi)
$$

(canonically attached to the weighted set $(S, \phi)$ ), then

$$
\mathcal{F}_{(S, \phi)}(u)=-\mathcal{E}_{\psi_{0}} \circ P_{S}(\phi-u) .
$$

This choice of reference $\psi_{0}$ ensures the normalization $\mathcal{F}_{(S, \phi)}(0)=0$. 
Priors leading to well-behaved Coulomb, Riesz gases vs zeroth-order phase transitions

Proposition 2.10. The functional $\mathcal{F}_{(S, \phi)}$ is convex and Gateaux differentiable on $\mathcal{C}(S)$ and its differential at $u$ is represented by the measure $\Delta\left(P_{S}(\phi-u)\right)$, i.e.

$$
{\frac{d \mathcal{F}_{(S, \phi)}(u+t v)}{d t}}_{\mid t=0}=\int v \Delta\left(P_{S}(\phi-u)\right)
$$

for any $v \in C(S)$.

Proof. This can be shown directly using the orthogonality relation (2.14) (as in the complex geometric setting in [11], which covers the logarithmic case $d=\alpha=2$ ). Alternatively, by Theorem 2.12 below $\mathcal{F}_{(S, \phi)}$ is the Legendre-Fenchel transform of the strictly convex functional $E_{\omega_{\phi}}$. Hence, by basic convex duality theory $\mathcal{F}_{(S, \phi)}$ is Gateaux differentiable and the differential at $u$ is the minimizer of $E_{\omega_{\phi-u}}$, i.e. $\mu_{\phi-u}$, which is equal to $\Delta P_{S}(\phi-u)$, by Prop 2.6.

\subsection{Energy and Legendre transforms}

In this section we assume that $\alpha \leq 2$ or (so that the domination principle (2.6) applies) and show that the functional $\mathcal{F}_{(S, \phi)}$ can be viewed as a Legendre-Fenchel transform of $E_{\omega_{\phi}}$.

Lemma 2.11. Assume that, on $S, \phi=\psi_{\nu}$, for a probability measure $\nu$ on $S$ (which implies that the canonical reference weight $\psi_{0}:=P_{S} \phi$ coincides with $\left.\psi_{\nu}\right)$. Then, for any $\mu \in \mathcal{P}_{S}\left(\mathbb{R}^{d}\right)$ such that $E(\mu)<\infty$, the corresponding normalized energy functional is given by

$$
E_{\omega_{\phi}}(\mu)=\mathcal{E}_{\psi_{0}}\left(\psi_{\mu}\right)-\int\left(\psi_{\mu}-\psi_{0}\right) \mu
$$

Proof. First rewrite

$$
\mathcal{E}_{\psi_{0}}(\psi)=\frac{1}{2} \int\left(\psi-\psi_{0}\right) \Delta\left(\psi-\psi_{0}\right)+\int\left(\psi-\psi_{0}\right) \Delta \psi_{0}
$$

Next, note that, in general, if $\mu$ and $\nu$ have finite energy, then there exists a constant $C_{1}$, depending on $\nu$, such that

$$
\frac{1}{2} \int\left(\psi_{\mu}-\psi_{\nu}\right) \Delta\left(\psi_{\mu}-\psi_{\nu}\right)=\frac{1}{2} \int \psi_{\mu} \Delta \psi_{\mu}-\int \psi_{\mu} \Delta \psi_{\nu}+C_{1}=:-E_{\phi}(\mu)+C_{1}
$$

using the symmetry (2.2) in the first equality. This shows that formula (2.18) holds up to an over all constant $C_{2}$. But the rhs in the formula vanishes for $\psi=\psi_{\nu}$ and so does $E_{\omega_{\phi}}(\nu)$, i.e. the minimum of $E_{\omega_{\phi}}(\mu)$ is realized for $\mu=\nu$, as follows from "completing the square". Hence, $C_{2}=0$, as desired.

Before stating the next theorem we recall the general definition of the LegendreFenchel transform. Let $f$ be a function on a topological vector space $V$. The LegendreFenchel transform $\widehat{f}$ of $f$ is defined as following convex lower semi-continuous function $\widehat{f}$ on the topological dual $V^{*}$

$$
\widehat{f}(w):=\sup _{v \in V}\langle v, w\rangle-f(v)
$$

in terms of the canonical pairing between $V$ and $V^{*}$. In the present setting we will take $V=\mathcal{C}(S)$ and $V^{*}=\mathcal{M}(S)$, the space of all continuous functions and the space of all signed Borel measures, respectively, on a compact topological space $S$. Then the Legendre-Fenchel transform is involutive [25]. Given a compact subset $S$ of $\mathbb{R}^{d}$ we will denote by $\chi_{\mathcal{P}(S)}$ the lsc functional on the space of all signed measures $\mathcal{M}(S)$ on $S \Subset \mathbb{R}^{d}$ which is equal to 0 on $\mathcal{P}(S)$ and equal to $\infty$ on the complement of $\mathcal{P}(S)$ in $\mathcal{M}(S)$. The definition is made so that the functional $\chi_{\mathcal{P}(S)}+E_{\left(S, \omega_{\phi}\right)}$ on $\mathcal{M}(S)$ is equal to $E_{\left(S, \omega_{\phi}\right)}$ if $\mu \in \mathcal{P}(S)$ and otherwise equal to $\infty$. 
Priors leading to well-behaved Coulomb, Riesz gases vs zeroth-order phase transitions

Theorem 2.12. Let $S$ be a compact subset of $\mathbb{R}^{d}$ and $\phi$ a continuous function on $S$. Consider the functional $\chi_{\mathcal{P}(S)}+E_{\omega_{\phi}}$ on the space $\mathcal{M}(S)$ of all signed measures on $S$. Its Legendre-Fenchel transform is given by

$$
\chi_{\mathcal{P}(S)+E_{\omega_{\phi}}}=\mathcal{F}_{(S, \phi)},
$$

where $\mathcal{F}_{(S, \phi)}$ is the functional defined in formula (2.17). Conversely,

$$
\chi_{\mathcal{P}(S)}+E_{\omega_{\phi}}=\widehat{\mathcal{F}(S, \phi)}
$$

Moreover, for any $\mu \in \mathcal{P}(S)$ such that $E(\mu)<\infty$

$$
E_{\omega_{\phi}}(\mu)=\mathcal{E}_{P_{S} \phi}\left(\psi_{\mu}\right)-\int\left(\psi_{\mu}-\phi\right) \mu
$$

Proof. In order to prove (2.20) and (2.21) it is, since the Legendre-Fenchel transform is involutive on $\mathcal{M}(S)$, enough to prove (2.20), or equivalently that, for any given $u \in \mathcal{C}(S)$,

$$
\inf _{\mathcal{P}(S)}\left(E_{\omega_{\phi}}(\mu)+\langle u, \mu\rangle\right)=\mathcal{E}_{P_{S} \phi}\left(P_{S}(\phi+u)\right) .
$$

Setting $\Phi=\phi+u$ and using that the inf above is attained at $\mu=\Delta\left(P_{S} \Phi\right)$ (by Prop 2.6) gives

$$
\inf _{\mathcal{P}(S)}\left(E_{\phi}(\mu)+\langle u, \mu\rangle\right)=E_{\phi}\left(\Delta\left(P_{S} \Phi\right)\right)+\left\langle\Phi-\phi, \Delta\left(P_{S} \Phi\right)\right\rangle .
$$

Rewriting the first term in the rhs as

$$
E_{\phi}\left(\Delta\left(P_{S} \Phi\right)\right)=E_{P \phi}\left(\Delta\left(P_{S} \Phi\right)\right)+\left\langle\left(\phi-P_{S} \phi\right), \Delta\left(P_{S} \Phi\right)\right\rangle
$$

yields

$$
\inf _{\mathcal{P}(S)}\left(E_{\omega_{\phi}}(\mu)+\langle u, \mu\rangle\right)=\left(E_{P_{S} \phi}\left(\Delta\left(P_{S} \Phi\right)\right)-E_{\phi}\left(\left(\Delta\left(P_{S} \phi\right)\right)\right)+\left\langle\Phi-P_{S} \phi, \Delta\left(P_{S} \Phi\right)\right\rangle .\right.
$$

Using the orthogonality relation in Prop 2.6 reveals that $E_{\phi}\left(\left(\Delta\left(P_{S} \phi\right)\right)=E_{P \phi}\left(\left(\Delta\left(P_{S} \phi\right)\right)\right.\right.$. Hence, by the previous lemma the first term in the rhs of the previous equation may be expressed as

$$
E_{P_{S} \phi}\left(\Delta\left(P_{S} \Phi\right)\right)=\mathcal{E}_{P_{S} \phi}(\Phi)-\left\langle\left(\Phi-P_{S} \phi\right), \Delta\left(P_{S} \Phi\right)\right\rangle
$$

Since the second term in the latter equation cancels the second term in former equation we thus get

$$
\inf _{\mathcal{P}(S)}\left(E_{\omega_{\phi}}(\mu)+\langle u, \mu\rangle\right)=\mathcal{E}_{P_{S} \phi}(\Phi)
$$

which coincides the right hand side in formula (2.23).

To prove the final statement we proceed essentially as above. First observe that, by definition,

$$
E_{\phi}(\mu)=E_{P_{S} \phi}(\mu)+\left\langle\phi-P_{S} \phi, \mu\right\rangle .
$$

Hence, applying the previous lemma to the weight $P_{S} \phi$, shows that there exists a constant $C$ (only depending on $\phi$ ) such that

$$
\begin{gathered}
E_{\omega_{\phi}}(\mu)=\mathcal{E}_{P_{S} \phi}\left(\psi_{\mu}\right)-\left\langle\psi_{\mu}-P_{S} \phi, \mu\right\rangle+\left\langle\phi-P_{S} \phi, \mu\right\rangle+C= \\
=\mathcal{E}_{P_{S} \phi}\left(\psi_{\mu}\right)-\left\langle\psi_{\mu}-\phi, \mu\right\rangle+C .
\end{gathered}
$$

Now, evaluating the previous equality for $\mu=\Delta\left(P_{S} \phi\right)$ and using that $\psi_{\mu}-P_{S} \phi$ is constant, by Prop 2.6 (so that we can replace $\psi_{\mu}$ with $P_{S} \phi$ in the rhs of the previous equation, using item 7 in Prop 2.8) gives

$$
0=0-\left\langle P_{S} \phi-\phi, \Delta\left(P_{S} \phi\right)\right\rangle+C .
$$

Finally, by the orthogonality relation in Prop 2.6, it follows that $C=0$, which concludes the proof of the theorem. 
Priors leading to well-behaved Coulomb, Riesz gases vs zeroth-order phase transitions

\subsection{Regularity}

In this section we assume that $\alpha \leq 2$. A weighted set $(S, \phi)$ will be said to be regular if $P_{S} \phi \leq \phi$ and a set $S$ is said to be regular if $(S, 0)$ is regular. A compact set $K$ is said to be locally regular if it is regular at any point $x \in K$, i.e. if $\left(P_{K \cap U} 0\right)(x) \leq 0$ for any open ball $U$ centered at $x$. In general, $P_{S} \phi \leq \phi$ always holds in the interior of $S$ (see the beginning of the proof of Lemma 2.15).

Lemma 2.13. A non-polar weighted compact set $(S, \phi)$ is regular iff $\sup _{S}\left(P_{S} \phi-\phi\right)=0$ iff $P_{S} \phi=\Pi_{S} \phi$ iff $P_{S} \phi$ is continuous.

Proof. The first equivalence follows from the extremal definition of $P_{S} \phi$, combined with Lemma 2.5 and Prop 2.6. To prove the second equivalence we note that if $(S, \phi)$ is regular, then $P_{S} \phi$ is a candidate for the sup defining $\Pi_{S} \phi$ and hence $P_{S} \phi \leq \Pi_{S} \phi$. Since the reverse inequality always holds we conclude that $P_{S} \phi=\Pi_{S} \phi$. Finally, let us show $(S, \phi)$ is regular iff $P_{S} \phi$ is continuous. First assume that $(S, \phi)$ is regular. By the previous step $P_{S} \phi=\Pi_{S} \phi$. Now, $P_{S} \phi$ is, by construction, usc. Hence, $\Pi_{S} \phi$ is continuous iff it is lsc. Accordingly, to prove that $\Pi_{S} \phi$ is continuous it is enough to show the following claim: the sup defining $\Pi_{S} \phi$ can be taken over all continuous $\psi \in \mathcal{L}_{c}\left(\mathbb{R}^{d}\right)$ satisfying $\psi \leq \phi$. To this end first note that there exists a sequence $\psi_{j} \in \mathcal{L}_{c}\left(\mathbb{R}^{d}\right) \cap C\left(\mathbb{R}^{d}\right)$ such that $\psi_{j} \rightarrow P_{S} \phi$ in $L_{l o c}^{1}$ and such that $\psi_{j}(x) \rightarrow P_{S} \phi(x)$ for any $x$ (as follows from [42, Thm 1.11 or Thm 3.7]). Moreover, $\psi_{j}$ may be taken to in $\mathcal{L}_{K}\left(\mathbb{R}^{d}\right)$ for some compact set $K$ containing $S$. Now, since $(S, \phi)$ is regular Prop 3.6 gives that the functional $\psi \mapsto \sup _{S}(\psi-\phi)$ is continuous on $\mathcal{L}_{K}\left(\mathbb{R}^{d}\right)$. Hence, replacing $\psi_{j}$ with $\tilde{\psi}_{j}:=\psi_{j}-\sup _{S}\left(\psi_{j}-\phi\right)$ and using that $\sup _{S}\left(P_{S} \phi-\phi\right)=0$ we may as well assume that $\psi_{j} \leq \phi$. But then the point-wise convergence of $\psi_{j}$ towards $P_{S} \phi$ proves the claim. Hence, $P_{S} \phi$ is continuous. Conversely, if $P_{S} \phi$ is continuous, then $P_{S} \phi \leq \phi$ q.e on $S$ implies that $P_{S} \phi \leq \phi$ everywhere on $S$, which means that $(S, \phi)$ is regular.

Lemma 2.14. Let $K$ be a non-polar compact set $K$. Then $(K, \phi)$ is regular for any $\phi \in C(K)$ iff $K$ is locally regular.

Proof. This is shown as in the complex setting [48, Prop 6.1]. First assume that $K$ is locally regular. Since $\phi$ is continuous we have that $\phi \leq \phi(x)+\delta(\epsilon)$ on an open ball $B_{\epsilon}(x)$ of radius $\epsilon$ centered at $x$, where $\delta(\epsilon) \rightarrow 0$ as $\epsilon \rightarrow 0$. Hence,

$$
P_{K} \phi \leq P_{K \cap B_{\epsilon}(x)} \phi \leq P_{K \cap B_{\epsilon}(x)}(\phi(x)+\delta(\epsilon)) \leq P_{K \cap B_{\epsilon}(x)} 0+\phi(x)+\delta(\epsilon) .
$$

Letting $\epsilon \rightarrow 0$ thus gives $\left(P_{K} \phi\right)(x) \leq \phi(x)$, showing that $(K, \phi)$ is regular. Conversely, assume that $(K, \phi)$ is regular for all $\phi \in \mathcal{C}(K)$. Take a point $x \in X$ and an open ball $B$ centered at $x$. Define a function $\phi$ on $K$ by setting $\phi=0$ on $K \cap B$ and $\phi=P_{K \cap B} 0$ on $K-B$. The function $\phi$ is clearly usc and hence there exists a sequence $\phi_{j} \in \mathcal{C}(K)$ decreasing to $\phi$. Now, if $\psi$ is candidate for the sup defining $\Pi_{K \cap B} 0$, then $\psi \leq 0$ on $K \cap B$ and hence $\psi \leq \Pi_{K \cap B} 0$ everywhere. As a consequence, $\psi \leq \phi$ on $K$, which, in turn, implies $\psi \leq P_{K} \phi_{j} \leq \phi_{j}$ for any $j$, using in the last equality that $\left(K, \phi_{j}\right)$ is assumed regular. Hence, taking the sup over all such $\psi$ and using that $\phi_{j}$ is continuous on $K$ gives $P_{K \cap B} 0 \leq \phi_{j}$. Finally, letting $j \rightarrow \infty$ we conclude that $P_{K \cap B} 0 \leq 0$ on $K \cap B$, as desired.

\subsubsection{Compact domains}

Now consider the case when $K$ is a compact domain, i.e. $K$ is the closure of an open bounded set $\Omega$. Following standard classical terminology $\Omega$ is said to be thin at $x_{0} \in \partial K:=K-\Omega$ if there exists some potential $\psi_{\mu}$ such that $\lim _{\sup } \operatorname{sux}_{x \rightarrow} \psi_{\mu}(x)<\psi_{\mu}\left(x_{0}\right)$, assuming $x \in \Omega$ (see the definition and discussion in [42, page 307]). 
Priors leading to well-behaved Coulomb, Riesz gases vs zeroth-order phase transitions

Lemma 2.15. Let $K$ be a compact domain, i.e. $K$ is the closure of an open bounded set $\Omega$. If $\Omega$ is non-thin at all boundary points, then $(K, \phi)$ is regular for any continuous $\phi$.

Proof. Set $\psi:=P_{K} \phi$. Then it follows from the continuity of $\phi$ that $\psi \leq \phi$ in $\Omega$. Indeed, by definition $\psi$ is the upper semi-continuous regularization $\left(\Pi_{K} \phi\right)^{*}$ of the function $\Pi_{K} \phi$, which satisfies $\Pi_{K} \phi \leq \phi$ on $K$. Thus, given $x \in \Omega$ and using that any ball $B_{\epsilon}(x)$ centered at $x$ of sufficiently small radius $\epsilon$ is contained on $\Omega$, we deduce that

$$
\psi(x):=\lim _{\epsilon \rightarrow 0} \sup _{B_{\epsilon}(x)}\left(\Pi_{K} \phi\right) \leq \lim _{\epsilon \rightarrow 0} \sup _{B_{\epsilon}(x)} \phi=\phi(x),
$$

since $\phi$ is continuous. Finally, given $x_{0} \in K-\Omega$, the assumption of non-thinness implies that $\psi\left(x_{0}\right) \leq \lim \sup _{x \rightarrow x_{0}} \psi(x) \leq \phi\left(x_{0}\right)$ since $x \in \Omega$ and $\phi$ is continuous.

The notion of thinness of a set $E$ at a point $x_{0}$ can equivalently be formulated in terms of Wiener's capacity criterion (see [42, Thm 5.2] and [42, Thm 5.10]), which, in turn, is equivalent to the following capacity criterion ([42, formula 5.1.7]): for a given number $q \in] 0,1[$,

$$
\sum_{m=1}^{\infty} \frac{\mathcal{C}_{\alpha}\left(E^{(m)}\right)}{q^{m(d-\alpha)}}<\infty, E^{(m)}:=E \cap\left\{x \in \mathbb{R}^{d}:\left|x-x_{0}\right|<q^{m}\right\},
$$

where $\mathcal{C}_{\alpha}$ denotes the capacity corresponding to $\alpha$ (recalled in Section 6.1 in the appendix). Here we have assumed that we are not in the logarithmic case $d=\alpha=2$, where a similar capicity criterion applies (see [42, Thm 5.6]).

Example 2.16. In the three-dimensional Coulomb case, $d=3$ and $\alpha=2$, algebraic cusps are non-thin at the vertex, while Lebesgue cusps are thin at the vertex [42, page 287]. These cusp as defined for a given $m>0$, by the surfaces of revolution in $\mathbb{R}^{3}$ where $\theta \leq r^{m}$ and $\theta \leq e^{-m / r}$ respectively (using planar polar coordinates). See also [37] for more general results in the Coulomb case.

For general $\alpha \in] 0,2]$ the capacity criterion above yields the following

Proposition 2.17. Let $K$ be a compact domain in $\mathbb{R}^{d}$ satisfying the interior cone condition, i.e. for any point $x_{0} \in \partial K$ there exists a cone contained in $K$ with a vertex at $x_{0}$. Then $K$ is locally regular.

Proof. By the previous lemma it is equivalent to show that $\left(P_{K} \phi\right)(x) \leq \phi(x)$ for any $\phi \in C(K)$ and $x \in K$. Given $x_{0} \in \partial K$ denote by $C_{x_{0}}$ a cone in $K$ with vertex at $x_{0}$. Note that it is enough to verify that interior of a cone $C_{x_{0}}$ is non-thin at the vertex. Indeed, since $\phi$ is continuous $P_{K} \phi \leq 0$ in the interior of $K$ and hence in the interior of $C_{x_{0}}$. Taking a sequence of points $x_{i}$ in the interior of $C_{x_{0}}$ converging towards $x_{0}$ and exploiting that $C_{x_{0}}$ is non-thin at $x_{0}$ it thus follows that $P_{S} \phi\left(x_{0}\right) \leq \lim \sup _{x \rightarrow x_{0}} P_{S} \phi(x) \leq \phi\left(x_{0}\right)$, using in the last inequality that $\phi$ is continuous. Finally, to verify that the interior of a compact cone $C_{x_{0}}$ is non-thin at $x_{0}$ first observe that, since $W_{\alpha}$ is translationally invariant, we may as well assume that $x_{0}$ is the origin. But then $E:=C_{x_{0}}$ has the scaling property that $E^{(m)}=q^{m} \cdot E^{(1)}$. It thus follows from the scaling properties of the kernel $W_{\alpha}$ that $\mathcal{C}_{\alpha}\left(E^{(m)}\right)=q^{m(d-\alpha)} \mathcal{C}_{\alpha}\left(E^{(1)}\right)$ and hence the capacity criterion $(2.24)$ is trivially satisfied (for notational simplicity we have assued that we are not in the logarithmic case $d=2=\alpha$, where essentially the same argument applies).

\subsection{Determining measures}

The definition of (strongly) determining measures was given in Section 1.1. It may be equivalently formulated as follows. A measure $\nu$ on $\mathbb{R}^{d}$ is said to be determining for a 
Priors leading to well-behaved Coulomb, Riesz gases vs zeroth-order phase transitions

weighted set $(S, \phi)$ if for all $\psi \in \mathcal{L}_{c}\left(\mathbb{R}^{d}\right)$

$$
\sup _{S} e^{\psi-\phi}=\left\|e^{\psi-\phi}\right\|_{L^{\infty}(S, \nu)}
$$

A measure $\nu$ is said to be determining for $S$ if $\nu$ is determining for $(S, 0)$ and strongly determining if $\nu$ is determining for $(S, \phi)$ for all $\phi \in C(S)$. Similarly we will say that $\nu$ is (strongly) determining if it is (strongly determining) for its support.

Proposition 2.18. If $\mu_{0}$ does not charge polar subsets, has compact support $S_{0}$ and is (strongly) determining, then $S_{0}$ is (locally) regular.

Proof. Since $\psi_{\phi}:=P_{S_{0}} \phi \leq \phi$ q.e. and $\mu_{0}$ does not charge polar subsets it follows that $\psi_{\phi} \leq \phi$ a.e. wrt $\mu_{0}$. By assumption this means that $\psi_{\phi} \leq \phi$ on $S_{0}$, i.e. $\left(S_{0}, \phi\right)$ is regular, as desired.

Any compact weighted regular compact subset carries determining measures:

Proposition 2.19. Let $(K, \phi)$ be a regular weighted compact set. Then the corresponding equilibrium measure $\mu_{(K, \phi)}$ is determining for $(K, \phi)$. As a consequence, if $\mu_{0}$ has the property that $\mu_{(K, \phi)}$ is absolutely continuous with respect to $\mu_{0}$, then $\mu_{0}$ is also determining for $(K, \phi)$.

Proof. Assume that $\psi \leq \phi$ a.e. wrt $\mu_{(K, \phi)}$. Recall that, by Prop 2.6, $\mu_{(K, \phi)}=\Delta\left(P_{K} \phi\right)$ and $\phi=P_{K} \phi$ a.e. wrt to $\Delta\left(P_{K} \phi\right)$. Hence, by the domination principle (2.6), $\psi \leq$ $P_{K} \phi$ everywhere. Finally, since $(K, \phi)$ is assumed regular it thus follows that $\psi \leq \phi$ everywhere, as desired.

This follows directly from the domination principle (2.6).

\subsubsection{Compact domains}

We will next consider the special case when $S_{0}$ is a compact domain (i.e. $S_{0}$ is the closure of an open bounded set), using the following lemma:

Lemma 2.20. Lebesgue measure $d x$ is strongly determining for any open subset $U \subset \mathbb{R}^{d}$. In other words, the measure $1_{U} d x$ is strongly determining.

Proof. Fix a smooth compactly supported function $\rho$ such that $\rho d x \in \mathcal{P}\left(\mathbb{R}^{n}\right)$ and set $\rho_{\delta}:=\delta^{n} \rho(x / \delta)$. Now, if $\psi_{\mu} \leq \phi$ a.e. on $U$, then, for any given compact subset $K$ of $U$, there exists a sequence $\epsilon_{j} \rightarrow 0$ such that $\psi_{j}:=\psi_{\mu} * \rho_{j^{-1}} \leq \phi+\epsilon_{j}$ on $K$. But $\psi_{j}=\psi_{\mu * \rho_{j}-1}$ and hence, by (2.5),

$$
\psi(x) \leq \limsup _{j \rightarrow \infty}\left(\phi(x)+\epsilon_{j}\right)=\phi(x)
$$

for any $x \in K$ and hence for any $x \in U$.

Note that in the Coulomb case, $\alpha=2$, the previous lemma follows directly from the submean property of subharmonic functions.

Proposition 2.21. Let $S_{0}$ be a compact domain, i.e. $S_{0}=\bar{\Omega}$, where $\Omega$ is an open bounded set. If $\Omega$ is non-thin at all boundary points, i.e. at all points in $X-\Omega$, then $\mu_{0}:=1_{\Omega} d x$ is strongly determining for $S_{0}$. In particular, if $S_{0}$ satisfies the interior cone condition (appearing in Prop 2.17), then $1_{\Omega} d x$ is strongly determining.

Proof. Assume that $\psi_{\mu} \leq \phi$ a.e. wrt $1_{\Omega} d x$. Then, by the previous lemma, $\psi_{\mu} \leq \phi$ on $\Omega$. Now take $x_{0} \in S_{0}-\Omega$. By the non-thinness assumption $\psi_{\mu}\left(x_{0}\right) \leq \lim \sup _{x \rightarrow x_{0}} \psi_{\mu}(x)$ for any sequence of points $x \in \Omega$ converging towards $x_{0}$. Since $\phi$ is continuous we deduce that $\psi_{\mu}\left(x_{0}\right) \leq \phi\left(x_{0}\right)$, showing that $\mu_{0}:=1_{\Omega} d x$ is strongly determining. 
Priors leading to well-behaved Coulomb, Riesz gases vs zeroth-order phase transitions

In particular, $1_{\Omega} d x$ is strongly determining if $\Omega$ is a bounded Lipschitz domain. Moreover, as shown next, the Hausdorff measure on the boundary $\partial \Omega$ of a bounded Lipschitz domain is strongly determining for $\partial \Omega$, in the Coulomb case.

\subsubsection{Lipschitz hypersurfaces}

Theorem 2.22. Consider the Coulomb case $\alpha=2$. The $(d-1)$-dimensional Hausdorff measure $\mu_{0}$ on a Lipschitz hypersurface $K$ in $\mathbb{R}^{d}$ without boundary is strongly determining.

Proof. Denote by $T$ be the closure of a bounded tubular neighborhood of $K$ and decompose it into two closed domains $T_{ \pm}$intersecting along $K$ :

$$
T=T_{-} \bigcup T_{+} .
$$

By assumption, the domains $T_{ \pm}$may be taken to be compact Lipschitz domains. Given a potential $\psi$ in $\mathbb{R}^{d}$ we fix a constant $C$ such that $\psi \leq C$ on $T$. Denote by $f_{ \pm}$the continuous function on $\partial T_{ \pm}$which is equal to a given continuous function $\phi$ on $K$ and equal to $C$ on $\partial T$. We denote by $h_{ \pm}$the harmonic extension of $f_{ \pm}$to $T_{ \pm}$. The function $h_{ \pm}$is in $\mathcal{C}\left(T_{ \pm}\right)$, as follows from the fact that $T_{ \pm}$satisfies the interior cone condition (see 2.17). Now, by assumption, $\psi \leq f_{ \pm}$almost everywhere with respect to the Hausdorff measure $\sigma_{ \pm}$on $\partial T_{ \pm}$. But then it follows from [27] that

$$
\psi \leq h_{ \pm} \text {, in the interior of } T_{ \pm} .
$$

Accepting, this for the moment and denoting by $h$ the continuous function on $T$ which is equal to $h_{ \pm}$on $T_{ \pm}$we get $\psi \leq h$ on $T$. Hence, since $\psi$ is subharmonic, for any $x \in K$ we have

$$
\psi(x) \leq \frac{1}{\left|B_{\delta}(x)\right|} \int_{B_{\delta}(x)} h d x
$$

Letting $\delta \rightarrow 0$ and using that $h$ is continuous and equal to $\phi(x)$ at $x$ we conclude that $\psi(x) \leq \phi(x)$, as desired.

Finally, we note that the inequality (2.25) is a standard consequence of the result in [27], saying, in particular, that for a Lipschitz domain $D$ the harmonic measure $\nu_{x}$ on $\partial D$ is absolutely continuous wrt the $(d-1)$-dimensional Hausdorff measure $\sigma$ on $\partial D$, for any $x \in \partial D$. Indeed, by the standard maximum principle for subharmonic functions

$$
\psi(x) \leq \int \nu_{x} \psi
$$

if $x$ is in the interior of $D$ (this is immediate in the case when $\psi$ is continuous in a neighborhood of $D$ and then general case then follows writing $\psi$ as a decreasing limit of such functions). Hence, applying (2.26) to $D=T_{ \pm}$and using that $\psi \leq f_{ \pm}$almost everywhere with respect $\sigma_{ \pm}$gives

$$
\psi(x) \leq \int \nu_{x} f_{ \pm}=h_{ \pm},
$$

proving (2.25).

Remark 2.23. The method of proof can be adapted to many other situations. Indeed, it only requires the existence of a neighborhood $T$ of $K$ such that the harmonic measures on the corresponding boundaries of $T_{ \pm}$are absolutely continuous wrt the corresponding Hausdorff measures. 


\section{Determining measures, Energy approximation and Gamma- convergence}

Given a probability measure $\mu_{0}$ with compact support $S_{0}$ and a continuous function $\phi$ on $\mathbb{R}^{d}$ the corresponding free energy functional $F_{\phi, \beta}$ at inverse temperature $\left.\left.\beta \in\right] 0, \infty\right]$ is defined by the following functional on $\mathcal{P}_{c}\left(\mathbb{R}^{d}\right)$ :

$$
F_{\phi, \beta}(\mu)=E_{\phi}(\mu)+\frac{1}{\beta} D_{\mu_{0}}(\mu), E_{\phi}(\mu):=E(\mu)+\int \phi \mu
$$

where $D_{\mu_{0}}$ denotes the entropy of $\mu$ relative to $\mu_{0}$ (also known as the Kullback-Leibler Divergence), i.e.

$$
D_{\mu_{0}}(\mu):=\int_{\mathbb{R}^{d}} \log \frac{\mu}{\mu_{0}} \mu
$$

when $\mu$ is absolutely continuous wrt to $\mu_{0}$ and otherwise $D_{\mu_{0}}(\mu):=\infty$. We define $F_{\phi, \infty}:=E_{\phi}$. Note that

$$
F_{\phi, \beta} \geq E_{\phi},
$$

since $D_{\mu_{0}} \geq 0$. We also recall that the functional $D_{\mu_{0}}$ is lower semi-continuous (lsc) on $\mathcal{P}(K)$, for any given compact subset $K$ [25].

Similarly, when replacing the energy $E_{\phi}$ with its normalized version $E_{\omega_{\phi}}(2.10)$ we will write

$$
F_{\omega_{\phi}, \beta}:=E_{\omega_{\phi}}(\mu)+\frac{1}{\beta} D_{\mu_{0}}(\mu)=F_{\phi, \beta}-\inf _{\mathcal{P}\left(S_{0}\right)} E_{\phi},
$$

where $S_{0}$ denotes the support of $\mu_{0}$. When $\phi=0$ we will simply use the notation $F_{\beta}:=F_{\phi, \beta}=F_{0, \beta}$.

\subsection{The Energy Approximation property vs Gamma-convergence of free ener- gies}

We recall the definition of Gamma-convergence, introduced by De Georgi (see the book [21] for background on Gamma-convergence):

Definition 3.1. A family of functions $F_{\beta}$ on a topological space $\mathcal{M}$ is said to Gammaconverge to a function $F$ on $\mathcal{M}$, as $\beta \rightarrow \infty$, if

$$
\begin{array}{ccc}
\mu_{\beta} \rightarrow \mu \text { in } \mathcal{M} & \Longrightarrow & \liminf _{\beta \rightarrow \infty} F_{\beta}\left(\mu_{\beta}\right) \geq F(\mu) \\
\forall \mu & \exists \mu_{\beta} \rightarrow \mu \text { in } \mathcal{M}: & \lim _{\beta \rightarrow \infty} F_{\beta}\left(\mu_{\beta}\right)=F(\mu)
\end{array}
$$

A sequence (family) $\mu_{\beta}$ as in the last point above is called a recovery sequence (family) for $\mu$. The limiting functional $F_{\infty}$ is automatically lower semi-continuous on $\mathcal{M}$.

We first make the following simple observation:

Lemma 3.2. A measure $\mu_{0}$ satisfies the Energy Approximation Property (section 1.1) iff the free energy $F_{\beta}$ Gamma-converges towards the energy $E$ on $\mathcal{P}(K)$.

Proof. First suppose that the Gamma-convergence holds. Given $\mu \in E(\mu)$ such that $E(\mu)<\infty$ we take a recovery family $\mu_{\beta}$, i.e.

$$
E(\mu) \geq \limsup _{\beta \rightarrow \infty} F_{\beta}\left(\mu_{\beta}\right)
$$

Since $F_{\beta} \geq E$ this directly implies the inequality (1.1) and hence the Energy Approximation Property. To prove the converse first observe that, since $E$ and $D_{\mu_{0}}$ are lsc on $\mathcal{P}(K)$ it is enough to show that for any $E(\mu)<\infty$ there exists a recovery family, which, in turn, is equivalent to finding a family $\mu_{\beta}$ such that $(i) \mu_{\beta} \rightarrow \mu$ in $\mathcal{P}(K),(i i) E\left(\mu_{\beta}\right) \rightarrow E(\mu)$, 
and $\beta^{-1} D_{\mu_{0}}\left(\mu_{\beta}\right) \rightarrow 0$. Moreover, the latter condition may be replaced by the condition that (iii) $D_{\mu_{0}}\left(\mu_{\beta}\right)<\infty$. Indeed, by relabeling the family $\mu_{\beta}$ we can then arrange that $D_{\mu_{0}}\left(\mu_{\beta}\right) \leq \beta^{1 / 2}$, say. Now, assuming that the energy approximation property holds, there exists a family $\mu_{\beta}$ satisfying the conditions $(i)$ and (ii) and such that $\mu_{\beta}=\rho_{\beta} \mu_{0}$ for some $\rho_{\beta} \in L^{1}\left(\mu_{0}\right)$. For any positive integer $j$ we set

$$
\mu_{\beta, j}:=\max \left(\rho_{\beta}, j\right) \mu_{0} / \int \max \left(\rho_{\beta}, j\right) \mu_{0} \in \mathcal{P}(K),
$$

which satisfies $D_{\mu_{0}}\left(\mu_{\beta, j}\right)<\infty$. Moreover, by the monotone convergence theorem, $E\left(\mu_{\beta, j}\right) \rightarrow E\left(\mu_{\beta}\right)$ and $\mu_{\beta, j} \rightarrow \mu_{\beta}$, as $j \rightarrow \infty$. Hence, we can conclude using a standard diagonal argument.

Gamma-convergence is stable under addition by continuous functionals, as follows directly from the definition. We will make use of the following criterion for Gammaconvergence on $\mathcal{P}(K)$, formulated in terms of the Legendre-Fenchel transform (Definition (2.19)):

Proposition 3.3. Let $F_{\beta}$ be a family of functions on the space $\mathcal{P}(K)$ of all probability measures on a compact space $K$ and extend $F_{\beta}$ by infinity to all of $\mathcal{M}(K)$. Assume that

$$
\lim _{\beta \rightarrow \infty} \widehat{F_{\beta}}(\phi)=f(\phi)
$$

for any $\phi \in C(X)$ and that $f$ defines a Gateaux differentiable function on $\mathcal{C}(K)$. Then $F_{\beta}$ Gamma-converges to $\widehat{f}$ on $\mathcal{M}(K)$ (the converse holds without any differentiability assumption).

See [6] for the proof of the previous proposition. Unraveling definitions reveals that, in the present setting, where $F_{\beta}$ is the free energy functional we have

$$
\lim _{\beta \rightarrow \infty} \widehat{F_{\beta}}(\phi)=\widehat{E}(\phi)
$$

iff

$$
\lim _{\beta \rightarrow \infty} \inf _{\mathcal{P}\left(S_{0}\right)} F_{\phi, \beta}=\inf _{\mathcal{P}\left(S_{0}\right)} E_{\phi}
$$

Thus in order to establish the Energy Approximation property, or equivalently, the Gamma-convergence of $F_{\beta}$ towards $E$, it is equivalent to establish the asymptotics above for the infima of $F_{\phi, \beta}$. for all continuous weights $\phi$.

Remark 3.4. Lemma 3.2 still holds if the entropy $D_{\mu_{0}}(\mu)$ is replaced by any lsc functional $\tilde{D}$ on $\mathcal{P}(K)$ with the property that $\tilde{D}(\mu)<\infty$ implies that $\mu$ is absolutely continuous with respect to $\mu_{0}$ and such that $\tilde{D}$ is finite on $L^{\infty}(K) \mu_{0}$ (using the same proof). But in the proof of Theorem 3.10 we will (implicitly) exploit that the Legendre-Fenchel transform of $\beta^{-1} D_{\mu_{0}}$ has good monotonicity and convergence properties with respect to $\beta$. Indeed, as is well-known, the Legendre-Fenchel transform of $\beta^{-1} D_{\mu_{0}}$ (extended by $\infty$ to the space $\mathcal{M}\left(S_{0}\right)$ of all signed measures on $\left.S_{0}\right)$ is the functional on $\mathcal{C}\left(S_{0}\right)$ defined by $u \mapsto \beta^{-1} \log \int e^{\beta u} \mu_{0}$, which increases to $\sup _{X} u$ as $\beta \rightarrow \infty$. But the actual proof of Theorem 3.10 does not explicitly invoke the Legendre-Fenchel transform, since we will need to allow $u$ to be non-continuous, namely of the form $\psi-\phi$, where $\psi$ is a potential.

\subsection{Determining measures vs Gamma-convergence of the free energies}

In this section we will assume that $\alpha \leq 2$ (so that the domination principle (2.6) applies). 
Priors leading to well-behaved Coulomb, Riesz gases vs zeroth-order phase transitions

Lemma 3.5. Assume given $\mu_{0}$ in $\mathcal{P}\left(\mathbb{R}^{d}\right)$ not charging polar subsets and of compact support $S_{0}$. Then, for any $\phi \in C\left(\mathbb{R}^{d}\right)$ and $\left.\beta \in\right] 0, \infty[$, the corresponding free energy functional $F_{\phi, \beta}$ on $\mathcal{P}\left(S_{0}\right)$ admits a unique minimizer $\mu_{\phi, \beta}$. Moreover, $\mu_{\phi, \beta}=\Delta \psi_{\phi, \beta}$, where $\psi_{\phi, \beta}$ is the unique solution in $\mathcal{E}_{S_{0}}\left(\mathbb{R}^{d}\right)$ of the following equation:

$$
\Delta \psi=e^{\beta(\psi-\phi)} \mu_{0}
$$

and we have

$$
\inf _{\mathcal{M}\left(S_{0}\right)} F_{\omega_{\phi}, \beta}=\sup _{\mathcal{E}_{c}\left(\mathbb{R}^{d}\right)} \mathcal{G}_{\phi, \beta}=\sup _{\mathcal{E}_{S_{0}}\left(\mathbb{R}^{d}\right)} \mathcal{G}_{\phi, \beta}=\mathcal{G}_{\phi, \beta}\left(\psi_{\phi, \beta}\right)
$$

where $\mathcal{G}_{\phi, \beta}$ is the following functional on $\mathcal{L}_{S}\left(\mathbb{R}^{d}\right)$, taking values in $[-\infty, \infty[$ :

$$
\mathcal{G}_{\phi, \beta}(\psi):=\mathcal{E}_{\psi_{0}}(\psi)-\mathcal{I}_{\beta}(\psi), \quad \psi_{0}:=P_{S_{0}}(\phi)
$$

where

$$
\mathcal{I}_{\beta}(\psi):=\beta^{-1} \log \int e^{\beta(\psi-\phi)} \mu_{0}
$$

(where we have suppressed the dependence on $\phi$ in the notation $\mathcal{I}_{\beta}$ ).

Proof. Step 1: $\sup _{\mathcal{E}_{c}\left(\mathbb{R}^{d}\right)} \mathcal{G}_{\phi, \beta}=\sup _{\mathcal{E}_{S_{0}}\left(\mathbb{R}^{d}\right)} \mathcal{G}_{\phi, \beta}=\mathcal{G}_{\phi, \beta}\left(\psi_{\phi, \beta}\right)$

To simplify the notation we will write $\mathcal{E}_{\psi_{0}}=\mathcal{E}$. First observe that $\mathcal{I}_{\beta}(\psi)>-\infty$ on $\mathcal{L}_{c}\left(\mathbb{R}^{d}\right)$. Indeed, if $\mathcal{I}_{\beta}(\psi)=-\infty$ then $\mu_{0}$ charges the polar set $\{\psi=-\infty\}$, which contradicts the assumption on $\mu_{0}$. Now fix any compact set $S$ containing $S_{0}$ and consider the functional $\mathcal{G}_{\phi, \beta}$ on $\mathcal{L}_{S}\left(\mathbb{R}^{d}\right)$. We note that $\mathcal{G}_{\beta}$ is usc. Indeed, by Prop $2.8 \mathcal{E}$ is usc and so is $-\mathcal{I}_{\beta}$, by Fatou's lemma. Moreover, $\mathcal{G}_{\phi, \beta}(\psi+c)=\mathcal{G}_{\phi, \beta}(\psi)$ and hence it follows from the compactness in Prop 2.2 that $\mathcal{G}_{\beta}$ admits a maximizer $\psi_{\beta}$. Since $\mathcal{I}_{\beta}(\psi)>-\infty$ we have $\psi_{\beta} \in \mathcal{E}_{S}\left(\mathbb{R}^{d}\right)$. All that remains is to verify that $\psi_{\beta}$ satisfies the equation (3.4) (after perhaps shifting $\psi_{\beta}$ by a constant). To this end fix a continuous bounded function $u, u \in \mathcal{C}_{b}\left(\mathbb{R}^{d}\right)$, and set

$$
g(t):=\mathcal{E}\left(P_{S}\left(\psi_{\beta}+t u\right)\right)-\mathcal{I}_{\beta}\left(\psi_{\beta}+t u\right) .
$$

The maximum of the function $g$ is attained at $t=0$. Indeed, for any $\psi \in \mathcal{E}_{S}\left(\mathbb{R}^{d}\right)+\mathcal{C}\left(S_{0}\right)$ we have that $\mathcal{I}_{\beta}\left(P_{S} \psi\right) \leq \mathcal{I}_{\beta}(\psi)$, using that $P_{S} \psi \leq \psi$ q.e. on $S$ and hence a.e. with respect to $\mu_{0}$ (since $\mu_{0}$ does not charge polar subsets). As a consequence,

$$
\mathcal{E}\left(P_{S}(\psi)\right)-\mathcal{I}_{\beta}(\psi) \leq \mathcal{E}\left(P_{S}(\psi)\right)-\mathcal{I}_{\beta}\left(P_{S}(\psi)\right):=\mathcal{G}_{\beta}\left(P_{S} \psi\right)
$$

In particular, since $P_{S} \psi \in \mathcal{E}_{S}\left(\mathbb{R}^{d}\right)$,

$$
\sup _{\psi \in \mathcal{E}_{S}\left(\mathbb{R}^{d}\right)+C\left(S_{0}\right)}\left(\mathcal{E}\left(P_{S}(\psi)\right)-\mathcal{I}_{\beta}(\psi)\right) \leq \sup _{\psi \in \mathcal{E}_{S}\left(\mathbb{R}^{d}\right)} \mathcal{G}_{\phi, \beta}=\mathcal{G}_{\phi, \beta}\left(\psi_{\phi, \beta}\right) .
$$

Thus, restricting $\psi$ in the lhs above to be in $\psi_{\beta}+\mathbb{R} u$ shows that the maximum of the function $g$ is, indeed, attained at $t=0$. Moreover, by Prop $2.10 g(t)$ is differentiable and hence $g^{\prime}(0)=0$ shows, using that $P_{S} \psi=\psi$, that the equation (3.4) holds when integrated against any $u \in \mathcal{C}_{b}\left(\mathbb{R}^{d}\right)$. Since a probability measure is uniquely determined by its action on $C_{b}\left(\mathbb{R}^{d}\right)$ this conclude the proof of Step 1.

Step 2: $\inf _{\mathcal{M}\left(S_{0}\right)} F_{\omega_{\phi}, \beta}=\sup _{\mathcal{E}_{S_{0}}\left(\mathbb{R}^{d}\right)} \mathcal{G}_{\phi, \beta}$, where the infimum is realized precisely at $\Delta \psi_{\phi, \beta}$

Since $E_{\omega_{\phi}}$ is convex and $D_{\mu_{0}}$ is strictly convex on the subset $\left\{D_{\mu_{0}}<\infty\right\} \subset \mathcal{P}\left(S_{0}\right)$ (by Jensen's inequality) the functional $F_{\omega_{\phi}, \beta}$ has at most one minimizer. By the previous step it will thus be enough to show that $\mu_{\phi, \beta}:=\Delta \psi_{\phi, \beta}$ minimizes $F_{\omega_{\phi}, \beta}$. But this follows 
Priors leading to well-behaved Coulomb, Riesz gases vs zeroth-order phase transitions

directly from the fact that $-\left(\psi_{\mu}-\phi\right)$ is a subgradient for $E_{\phi}$ and $\log \left(\mu / \mu_{0}\right)$ is a subgradient for $D_{\mu_{0}}$ (by convexity). Finally, evaluating $F_{\omega_{\phi}, \beta}$ at $\mu_{\phi, \beta}$ and using formula (2.22) gives

$$
F_{\omega_{\phi}, \beta}\left(\mu_{\phi, \beta}\right)=\mathcal{E}_{P_{S} \phi}\left(\psi_{\phi, \beta}\right)-\int\left(\psi_{\phi, \beta}-\phi\right) \mu+\frac{1}{\beta} \int \log e^{\beta\left(\psi_{\phi, \beta}-\phi\right)}=\mathcal{E}_{P_{S} \phi}\left(\psi_{\phi, \beta}\right) .
$$

Finally, since $e^{\beta\left(\psi_{\phi, \beta}-\phi\right)}\left(=\Delta \psi_{\phi, \beta}\right)$ is a probability measure we have that $\mathcal{I}_{\beta}\left(\psi_{\beta, \phi}\right)=0$ and hence $\mathcal{E}_{P_{S} \phi}\left(\psi_{\phi, \beta}\right)=\mathcal{G}_{\phi, \beta}\left(\psi_{\phi, \beta}\right)$, which concludes the proof of Step 2 .

Step 3: The solution $\psi_{\phi, \beta}$ of equation (3.4) is uniquely determined.

By the previous step $\Delta\left(\psi_{\phi, \beta}\right)$ is the unique minimizer of $F_{\omega_{\phi}, \beta}$ on $\mathcal{P}\left(S_{0}\right)$. Hence, $\psi_{\phi, \beta}$ is uniquely determined up to an additive constant. But, since, as explained in the previous step, $\mathcal{I}_{\beta}\left(\psi_{\beta, \phi}\right)=0$ the constant in question vanishes.

We next establish an approximate reversed Hölder type inequality for measures $\mu_{0}$ not charging polar subsets. The result mimics the logarithmic case, which is covered by the complex-geometric setting in [12, Thm 1.14] and shows that $\mu_{0}$ is determining iff $\mu_{0}$ satisfies a potential-theoretic analog of the Bernstein-Markov inequality for polynomials:

Proposition 3.6. Assume that $\mu_{0}$ has compact support $S_{0}$ and does not charge polar subsets. Then the following is equivalent for a given continuous function $\phi$ :

- $\mu_{0}$ is determining for $\left(S_{0}, \phi\right)$

- For all $\epsilon>0$ there exist a constant $C$ such that

$$
\sup _{S_{0}} e^{\psi-\phi} \leq C^{1 / p} e^{\epsilon}\left\|e^{\psi-\phi}\right\|_{L^{p}\left(S_{0}, \mu_{0}\right)}
$$

for any $\psi \in \mathcal{L}_{K}\left(\mathbb{R}^{d}\right)$ and $p>0$.

As a consequence, if $K$ is compact and $(K, \phi)$ is regular then the functional

$$
\mathcal{L}_{K}(\psi):=\sup _{K}(\psi-\phi)
$$

is continuous on $\mathcal{L}_{S}\left(\mathbb{R}^{d}\right)$ for any given compact set $S$.

Proof. Given the general properties recalled in Section 2.1 and the compactness result in Prop 2.2 the proof follows, more or less verbatim, from the proof of the corresponding result in [12, Thm 1.14]. For completeness we provide the argument here.

Step 1: The functional $\mathcal{L}_{K}$ is usc on $\mathcal{L}_{K}\left(\mathbb{R}^{n}\right)$ for any compact set $K$.

This is shown exactly as in the case $\phi=0$ appearing in the proof of Step 2 in Prop 2.2.

Step 2: If $\mu_{0}$ does not charge polar sets, then the functional $\mathcal{I}_{p}$ (formula (3.7)) is continuous on $\mathcal{L}_{K}\left(\mathbb{R}^{n}\right)$ for any $p>0$.

If $\psi_{j}$ is a sequence of functions in $\mathcal{L}_{K}\left(\mathbb{R}^{n}\right)$ converging in $L_{l o c}^{1}$ towards $\psi$, then, by (2.8) and (2.4)

$$
\text { (i) } \sup _{K} \psi_{j} \leq C, \quad \lim \sup \psi_{j}=\psi \mu_{0}-\text { a.e }
$$

since $\mu_{0}$ does not charge polar sets. The continuity of the functional $\mathcal{I}_{p}$ now follows from a Hilbert space argument using convex combinations of $f_{j}:=e^{\psi_{j}-\phi}$, by repeating the argument in the proof of [12, Thm 1.14] word by word.

Step 3:In general, $\mathcal{I}_{p}$ is increasing in $p$ and

$$
\lim _{p \rightarrow \infty} \mathcal{I}_{p}(\psi)=\mathcal{L}_{\infty}(\psi):=\log \left\|e^{\psi-\phi}\right\|_{L^{\infty}\left(S, \mu_{0}\right)}
$$

Indeed, this follows from Hölder's inequality and standard integration theory. 
Priors leading to well-behaved Coulomb, Riesz gases vs zeroth-order phase transitions

Now, if $\mu_{0}$ does not charge polar sets, then, combining Step 2 and Step 3, reveals that the functional $\mathcal{L}_{\infty}$ is lsc. If $\mu_{0}$ is moreover determining then $\mathcal{L}_{\infty}=\mathcal{L}_{S_{0}}$ and hence $\mathcal{L}_{\infty}$ is also usc continuous by Step 1 and hence continuous. To conclude the proof of the inequality (3.8) it will be enough to show that $f_{p}:=\mathcal{I}_{p}-\mathcal{L}_{\infty}$ converges uniformly to 0 on $\mathcal{L}_{S_{0}}\left(\mathbb{R}^{d}\right)$. Since $f_{p}(\psi+c)=f_{p}(\psi)$ it is enough to prove this on the subspace of all meannormalized $\psi$. But since the latter space if compact (Prop 2.2) the uniform convergence in question follows from Step 3, using Dini's lemma. Conversely, if the inequality (3.8) holds, then letting $p \rightarrow \infty$ gives $\mathcal{L}_{S_{0}} \leq \mathcal{L}_{\infty}$ on $\mathcal{L}_{S_{0}}\left(\mathbb{R}^{d}\right)$, i.e. $\mu_{0}$ is determining (since trivially $\mathcal{L}_{\infty} \leq \mathcal{L}_{S_{0}}$ ).

Finally, the last statement in the proposition is obtained by taking $\mu_{0}$ to be the equilibrium measure of $(K, \phi)$ and using Prop 2.19.

We note that for any measurable function $u$ on a measure space $\left(S, \mu_{0}\right)$

$$
\sup _{\mu_{0}} u:=\log \left\|e^{u}\right\|_{L^{\infty}\left(S, \mu_{0}\right)},
$$

is called the essential sup of $u$ on $\left(S, \mu_{0}\right)$. Given a measure $\mu_{0}$ we now define the following function on $\mathbb{R}^{d}$, taking values in $\left.] 0, \infty\right]$ :

$$
\left(\Pi_{\mu_{0}} \phi\right)(x):=\sup _{\mathcal{L}_{S_{0}}\left(\mathbb{R}^{d}\right)}\left\{\psi(x): \sup _{\mu_{0}}(\psi-\phi) \leq 0\right\},
$$

where $S_{0}$ denotes the support of $\mu_{0}$. Its upper semi-continuous regularization is denoted by

$$
P_{\mu_{0}} \phi:=\left(\Pi_{\mu_{0}} \phi\right)^{*}
$$

This definition should be compared with definition of $P_{S} \phi$, in formula (2.12). However, in general, $P_{\mu_{0}} \phi$ can be different from $P_{S_{0}} \phi$, for the support $S_{0}$ of $\mu_{0}$ (unless $\mu_{0}$ is determining).

Remark 3.7. In the logarithmic case $d=\alpha=2$ the function $P_{\mu_{0}} \phi$ coincides with the minimal carrier Green function [55] when $\phi=0$. For a general $\phi$ it coincides with the quasi-plurisubharmonic envelope on Kähler manifolds $X$ introduced in [35] (specialized to the case when $X$ is the Riemann sphere).

Lemma 3.8. Let $\mu_{0}$ be a measure on $\mathbb{R}^{d}$ which does not charge polar subsets and with compact support $S_{0}$ and $\phi$ a continuous function on $\mathbb{R}^{d}$. Then

$$
P_{\mu_{0}} \phi \in \mathcal{E}_{S_{0}}\left(\mathbb{R}^{d}\right)
$$

and

$$
\sup _{\mu_{0}}\left(P_{\mu_{0}} \phi-\phi\right)=0
$$

Proof. Step 1: $\Pi_{\mu} \psi$ is locally bounded from above

Given a large ball $B$ it is enough to show the existence of a constant $C$ such that

$$
\delta(\psi):=\sup _{B}(\psi-\phi)-\sup _{\mu}(\psi-\phi) \leq C .
$$

By Step 1 in the proof of Prop 3.6 the first functional in the lhs above is usc on $\mathcal{L}_{S_{0}}\left(\mathbb{R}^{d}\right)$ for any compact set $B$. Moreover, as explained in the proof of Prop 3.6 the second functional is lsc for any measure $\mu_{0}$ not charging polar subsets. This means that the functional $\delta(\psi)$ is usc on $\mathcal{L}_{S_{0}}\left(\mathbb{R}^{d}\right)$ and satisfies $\delta(\psi+c)=\delta(\psi)$ for any $c \in \mathbb{R}$. By the compactness of the subspace of $\mathcal{L}_{S_{0}}\left(\mathbb{R}^{d}\right)$ consisting of mean-normalized functions this yields the existence of a constant $C$ as above.

Step 2: $P_{\mu_{0}} \phi \in \mathcal{E}_{S_{0}}\left(\mathbb{R}^{d}\right)$ and $\sup _{\mu_{0}}\left(P_{\mu_{0}} \phi-\phi\right)=0$ 
First we recall "Choquet's lemma": let $\left\{u_{\alpha}\right\}_{\alpha \in A}$ be a family of real valued functions on a metric separable space $X$ (that we shall take to be $\mathbb{R}^{d}$ ). Suppose furthermore that this family is locally bounded from above. Then there exists a countable subset $B$ of $A$ such that

$$
\left(\sup \left\{u_{\beta}\right\}_{\beta \in B}\right)^{*}=\left(\sup \left\{u_{\alpha}\right\}_{\alpha \in A}\right)^{*},
$$

where $\sup \left\{u_{\beta}\right\}$ denotes the function on $\mathbb{R}^{d}$ defined as the point-wise sup. Thus, by Choquet's lemma, there exists a countable family of functions $\psi_{i}$, which are candidates for the sup defining $P_{\mu} \phi$, satisfying

$$
\left(\sup \left\{\psi_{i}\right\}\right)^{*}=\left(P_{\mu} \phi\right) .
$$

Recall that, in general, a Borel subset $S \subset \mathbb{R}^{d}$ is called a $\mu_{0}$-carrier of a measure $\mu_{0}$ on $\mathbb{R}^{d}$ if $\mu(S)=\mu\left(\mathbb{R}^{d}\right)$, i.e. if $\mu_{0}\left(\mathbb{R}^{d}-S\right)=0$. Since $\sup _{\mu_{0}}\left(P_{\mu_{0}} \psi_{i}-\psi_{i}\right)=0$ we have that $\psi_{i} \leq \phi$ on a $\mu_{0}$-carrier $S_{i}$. Denote by $S$ the intersection of all $S_{i}$. Then $S$ is also a $\mu_{0}$-carrier. Take a subset $K_{\sigma} \Subset S$ which is a union of increasing compact subsets of $S$ such that $\mu_{0}\left(K_{\sigma}\right)=\mu_{0}(S)$ (the existence of $K_{\sigma}$ follows from the fact that a Borel measure $\mu_{0}$ is, in particular, interior regular). Since $\psi_{i} \leq \phi$ on $K_{\sigma}$ we have $\psi_{i} \leq P_{K_{\sigma}} \phi$. Moreover, by Prop 2.6, $P_{K_{\sigma}} \phi \in \mathcal{L}_{S_{0}}\left(\mathbb{R}^{d}\right)$ and $P_{K_{\sigma}} \phi \leq \phi$ q.e. on the $\mu_{0}$-carrier $K_{\sigma}$. Hence, $P_{\mu} \phi \leq$ $P_{K_{\sigma}} \phi \leq P_{\mu} \phi$, using in the last inequality that $P_{K_{\sigma}} \phi \leq \phi \mu_{0}$-almost everywhere, since $\mu_{0}$ does not charge polar sets. This shows that $P_{\mu} \phi \in \mathcal{E}_{S_{0}}\left(\mathbb{R}^{d}\right)$ and $\sup _{\mu_{0}}\left(P_{\mu_{0}} \phi-\phi\right) \leq 0$. But then the extremal definition of $\Pi_{\mu_{0}}$ forces $\sup _{\mu_{0}}\left(P_{\mu_{0}} \phi-\phi\right)=0$.

We shall also need the following

Lemma 3.9. Suppose that $K$ is compact and $(K, \phi)$ is regular. Then

$$
\inf _{\mathcal{P}(K)} E_{\omega_{\phi}}=\sup _{\psi \in \mathcal{L}_{c}\left(\mathbb{R}^{d}\right)} \mathcal{G}_{K}=\sup _{\psi \in \mathcal{L}_{K}\left(\mathbb{R}^{d}\right)} \mathcal{G}_{K}
$$

where

$$
\mathcal{G}_{K}(\psi):=\mathcal{E}(\psi)-\sup _{K}(\psi-\phi) .
$$

Moreover, $P_{K} \phi$ is the unique maximizer of the functional $\mathcal{G}_{K}$ subject to the normalization $\sup _{K}(\psi-\phi)=0$. Similarly, if $\mu_{0}$ has compact support and does not charge polar subsets, then $P_{\mu_{0}} \phi$ is the unique maximizer of the functional

$$
\mathcal{G}_{\infty}(\psi):=\mathcal{E}(\psi)-\sup _{\mu_{0}}(\psi-\phi),
$$

subject to the normalization $\sup _{\mu_{0}}(\psi-\phi)=0$.

Proof. By Theorem 2.12 the lhs in formula (3.10) is given by $\mathcal{E}\left(P_{K} \phi\right)$ which in turn is given by $\mathcal{G}_{K}\left(P_{K} \phi\right)$, by the regularity assumption. Moreover, if $\psi \in \mathcal{L}_{c}\left(\mathbb{R}^{d}\right)$ and $\sup _{K}(\psi-\phi)=0$, then $\psi \leq P_{K} \phi$ (by the very definition of $P_{K} \phi$ ) and hence $\mathcal{G}_{K}(\psi) \leq$ $\mathcal{G}_{K}\left(P_{K} \phi\right)$, since $\mathcal{E}$ is increasing (Prop 2.8). Moreover, the uniqueness in question follows from the fact that $\mathcal{E}$ is strictly increasing. The corresponding results for $\mathcal{G}_{\infty}(\psi)$ are shown in a similar way, now using that $\sup _{\mu_{0}}\left(P_{\mu_{0}} \phi-\phi\right)=0$, by the previous lemma.

We are now ready for the proof of the core analytic result of the present paper:

Theorem 3.10. Let $\mu_{0}$ be a measure on $\mathbb{R}^{d}$ which does not charge polar subsets and assume that $\mu_{0}$ has compact support $S_{0}$. Given a continuous function $\phi$ on $\mathbb{R}^{d}$ the following is equivalent:

(1) $\mu_{0}$ is determining for $\left(S_{0}, \phi\right)$ 
Priors leading to well-behaved Coulomb, Riesz gases vs zeroth-order phase transitions

(2) $\left(S_{0}, \phi\right)$ is regular and

$$
\lim _{\beta \rightarrow \infty} \inf _{\mathcal{P}\left(S_{0}\right)} F_{\phi, \beta}=\inf _{\mathcal{P}\left(S_{0}\right)} E_{\phi}
$$

(3) $\left(S_{0}, \phi\right)$ is regular and the minimizers $\mu_{\phi, \beta}$ of $F_{\phi, \beta}$ converge weakly towards the minimizer $\mu_{\left(S_{0}, \phi\right)}$ of $E_{\phi}$ as $\beta \rightarrow \infty$ (and then convergence in energy automatically holds)

(4) $\left(S_{0}, \phi\right)$ is regular and the solution $\psi_{\beta, \phi}$ of the equation (3.4) converges towards $P_{S_{0}} \phi$ in energy, as $\beta \rightarrow \infty$.

Proof. First note that since $F_{\phi, \beta}$ coincides with $F_{\omega_{\phi}, \beta}$ up to an additive constant, which is independent of $\beta$, it is equivalent to prove the theorem with $F_{\phi, \beta}$ and $E_{\phi}$ replaced by $F_{\omega_{\phi}, \beta}$ and $E_{\omega_{\phi}}$, respectively.

Step 1: 1 implies 2 and 3 and 4.

By Lemma 3.5

$$
\inf _{\mathcal{M}\left(S_{0}\right)} F_{\omega_{\phi}, \beta}=\sup _{\mathcal{E}_{S_{0}}\left(\mathbb{R}^{d}\right)} \mathcal{G}_{\phi, \beta}
$$

and hence, by the previous lemma, the convergence (3.13) may be reformulated as

$$
\lim _{\beta \rightarrow \infty} \sup _{\mathcal{L}_{S_{0}}\left(\mathbb{R}^{d}\right)} \mathcal{G}_{\beta}=\sup _{\psi \in \mathcal{L}_{S_{0}}\left(\mathbb{R}^{d}\right)} \mathcal{G}_{S_{0}}
$$

Now, if $\mu_{0}$ is determining for $\left(S, \phi_{0}\right)$, then, by Prop 3.6, for any $\epsilon>0$ there exists a constant $C$ such that

$$
\sup _{S_{0}}(\psi-\phi)-C / \beta-\epsilon \leq \mathcal{I}_{\beta}(\psi) \leq \sup _{S_{0}}(\psi-\phi)+C / \beta
$$

Since the functional $\mathcal{E}$ is usc this immediately implies the convergence in item 2. Moreover, by compactness (Prop 2.2) we may, after perhaps passing to a subsequence, assume that the maximizer $\psi_{\beta, \phi}$ of $\mathcal{G}_{\phi, \beta}$ converges towards a maximizer of $\mathcal{G}_{S_{0}}$. Hence, by the previous lemma, $\psi_{\beta, \phi}$ converges towards $P_{S_{0}} \phi$, which combined with (3.15) gives $\mathcal{E}\left(\psi_{\beta, \phi}\right) \rightarrow \mathcal{E}\left(P_{S_{0}} \phi\right)$. This implies (Prop 2.8) that $E_{\omega_{\phi}}\left(\mu_{\beta, \phi}\right) \rightarrow E_{\omega_{\phi}}\left(\Delta P_{S_{0}} \phi\right)=E_{\omega}\left(\mu_{\left(S_{0}, \phi\right.}\right)$ and hence 2,3 and 4 follow.

Step 2: 2 implies 1

First note that, since trivially, $\mathcal{I}_{\beta} \leq \mathcal{L}_{\infty} \leq \mathcal{L}_{S_{0}}$ we have that

$$
\liminf _{\beta \rightarrow \infty} \sup _{\mathcal{L}_{S_{0}}\left(\mathbb{R}^{d}\right)} \mathcal{G}_{\beta} \geq \sup _{\psi \in \mathcal{L}_{S_{0}}\left(\mathbb{R}^{d}\right)} \mathcal{G}_{\infty} \geq \sup _{\psi \in \mathcal{L}_{S_{0}}\left(\mathbb{R}^{d}\right)} \mathcal{G}_{S_{0}}
$$

Combined with (3.14) this means that if item 2 holds, then the inequalities above must be equalities and hence

$$
\sup _{\mathcal{L}_{S_{0}}\left(\mathbb{R}^{d}\right)} \mathcal{G}_{\infty}=\sup _{\psi \in \mathcal{L}_{S_{0}}\left(\mathbb{R}^{d}\right)} \mathcal{G}_{S_{0}}
$$

But this implies that $P_{\mu_{0}} \phi=P_{S_{0}} \phi$. Indeed, by definition, we have $P_{\mu_{0}} \phi \geq P_{S_{0}} \phi$ and since $\mathcal{L}_{S_{0}}\left(P_{S_{0}} \phi\right)=0=\mathcal{L}_{\infty}\left(P_{\mu_{0}} \phi\right)$ the equality (3.17) forces $\mathcal{E}\left(P_{\mu_{0}} \phi\right) \geq \mathcal{E}\left(P_{S_{0}} \phi\right)$. By the strict monotonicity of $\mathcal{E}$ this means that $P_{\mu_{0}} \phi=P_{S_{0}} \phi$. Since, by definition, $P_{\mu_{0}} \phi$ and $P_{S_{0}} \phi$ are defined as the upper semi-continuous regularizations of $\Pi_{\mu_{0}} \phi$ and $\Pi_{S_{0}} \phi$, respectively, it thus follows that

$$
\Pi_{\mu_{0}} \phi \leq\left(\Pi_{\mu_{0}} \phi\right)^{*}=\left(\Pi_{S_{0}} \phi\right)^{*} \leq \phi
$$

using that $\left(S_{0}, \phi\right)$ is assumed regular in the last equality. Hence, $\mu_{0}$ is determining for $\left(S_{0}, \phi\right)$.

Step 3: The weak convergence in item 3 implies convergence in energy and items 4 and 2 
Priors leading to well-behaved Coulomb, Riesz gases vs zeroth-order phase transitions

Assume that $\mu_{\phi, \beta}$ converges towards $\mu_{\left(S_{0}, \phi\right)}$. By compactness (Prop 2.2) this means that there exist constants $C_{\beta}$ such that

$$
\psi_{\beta, \phi}+C_{\beta} \rightarrow P_{S_{0}} \phi
$$

in $L_{\text {loc }}^{1}$. Since $\mathcal{L}_{\beta, \phi}\left(\psi_{\beta, \phi}\right)=0$ it follows that

$$
\lim _{\beta \rightarrow \infty} C_{\beta}=\lim _{\beta \rightarrow \infty} \mathcal{L}_{\beta, \phi}\left(P_{S_{0}} \phi\right)=\mathcal{L}_{\infty, \phi}\left(P_{S_{0}} \phi\right)=\mathcal{L}_{S_{0}, \phi}\left(P_{S_{0}} \phi\right)=0
$$

using in the next to last equality that $P_{S_{0}} \phi$ is continuous (by Lemma 2.13), since $\left(S_{0}, \phi\right)$ is assumed regular. Hence, $\psi_{\beta, \phi}$ converges towards $P_{S_{0}} \phi$ in $L_{l o c}^{1}$ and the lower bound (3.16) gives

$$
\liminf _{\beta \rightarrow \infty} \mathcal{E}\left(\psi_{\beta, \phi}\right) \geq \mathcal{E}\left(P_{S_{0}} \phi\right) .
$$

Since $\mathcal{E}$ is usc this shows that, in fact,

$$
\mathcal{E}\left(\psi_{\beta, \phi}\right) \rightarrow \mathcal{E}\left(P_{S_{0}} \phi\right) .
$$

Hence, item 4 holds. Now, by Lemma 3.5,

$$
\beta^{-1} D_{\mu_{0}}\left(\mu_{\beta, \phi}\right)=\int\left(\psi_{\beta, \phi}-\phi\right) \mu_{\beta, \phi} \rightarrow \int\left(P_{S_{0}} \phi-\phi\right) \Delta\left(P_{S_{0}} \phi\right)=0
$$

using (3.18) in the convergence step and the orthogonality relation (2.14) in the last equality. All in all this means the weak convergence in 3 implies the convergence in energy of $\mu_{\beta, \phi}$, as well as the convergence of free energies in item 2 .

Finally, combining the previous theorem with Prop 3.3 (and the subsequent discussion) and Lemma 3.2 we arrive at the following result, which contains, in particular, Theorem 1.1 and Theorem 1.2 stated in the introduction, except the LDP statement proved in Section 4.1.

Theorem 3.11. Let $\mu_{0}$ be a measure on $\mathbb{R}^{d}$ which does not charge polar subsets and assume that the support $S_{0}$ of $\mu_{0}$ is compact. Then the following is equivalent:

(1) The measure $\mu_{0}$ is strongly determining

(2) $S_{0}$ is locally regular and $\inf _{\mathcal{P}\left(S_{0}\right)} F_{\phi, \beta} \rightarrow \inf _{\mathcal{P}\left(S_{0}\right)} E_{\phi}$, as $\beta \rightarrow \infty$, for any given $\phi \in C\left(S_{0}\right)$.

(3) $S_{0}$ is locally regular and the functional $F_{\beta}$ converges towards $E$, as $\beta \rightarrow \infty$, in the sense of Gamma-convergence.

(4) $S_{0}$ is locally regular and the measure $\mu_{0}$ has the Energy Approximation Property.

(5) $S_{0}$ is locally regular and for any given $\phi \in \mathcal{C}\left(S_{0}\right)$ the measures $\mu_{\phi, \beta}$ converge weakly towards $\mu_{\left(S_{0}, \phi\right)}$, as $\beta \rightarrow \infty$ (and then convergence in energy automatically holds)

\subsection{Quasi-explicit approximations using finite energy weights $\phi$}

In this section we provide a constructive procedure for obtaining the approximation in Theorem 1.1. To this end we assume, as before, that $\alpha \leq 2$ and consider generalized weights

$$
\phi \in \mathcal{C}(S)+\mathcal{E}_{S}\left(\mathbb{R}^{d}\right)-\mathcal{E}_{S}\left(\mathbb{R}^{d}\right) .
$$

(for the construction in question it is enough to work with $\mathcal{C}(S)+\mathcal{E}_{S}\left(\mathbb{R}^{d}\right)$, but since it requires not extra effort we will consider the more general setting). First recall that by basic Hilbert space duality theory we have the following 
Priors leading to well-behaved Coulomb, Riesz gases vs zeroth-order phase transitions

Lemma 3.12. Let $S$ be a compact subspace of $\mathbb{R}^{d}$. Then a function $\phi$ is in $\mathcal{E}_{S}\left(\mathbb{R}^{d}\right)$ iff $|\langle\phi, \mu\rangle|<\infty$ for all measures $\mu \in \mathcal{P}(S)$ satisfying $E(\mu)<\infty$. Moreover, if $\phi \in \mathcal{E}_{S}\left(\mathbb{R}^{d}\right)$, then the functional $\langle\phi, \cdot\rangle$ is continuous wrt the weak topology on any sublevel set $\{E \leq C\}$ in $\mathcal{P}(S)$.

Now, given a generalized weight $\phi \in \mathcal{C}(S)+\mathcal{E}_{S}\left(\mathbb{R}^{d}\right)-\mathcal{E}_{S}\left(\mathbb{R}^{d}\right)$ we define the corresponding weighted energy by

$$
E_{\phi}(\mu):=E(\mu)+\langle\phi, \mu\rangle
$$

if $E(\mu)<\infty$ and otherwise $E_{\phi}(\mu):=\infty$.

Lemma 3.13. Let $S$ be a non-polar subset. Then the restriction of $E_{\phi}$ to $\mathcal{P}(S)$ is lsc and strictly convex.

Proof. This is shown using Hilbert space theory exactly as in the proof of [10, Thm 3.21] (which concerns the case when $d=\alpha=2$ ).

In particular, if $S$ is a non-polar compact set, then $E_{\phi}$ admits a unique minimizer on $\mathcal{P}(X)$ that we shall call denote, as before, by $\mu_{(S, \phi)}$. Combining the previous two lemmas yields the following generalization of the convergence in item 5 of Theorem 3.11 to generalized weights $\phi$ :

Proposition 3.14. Let $\mu_{0}$ be a measure in $\mathbb{R}^{d}$ with compact support $S_{0}$ such that $\mu_{0}$ does not charge polar sets and $\mu_{0}$ is determining and assume that

$$
\phi \in \mathcal{C}\left(S_{0}\right)+\mathcal{E}_{S_{0}}\left(\mathbb{R}^{d}\right)-\mathcal{E}_{S_{0}}\left(\mathbb{R}^{d}\right) .
$$

Then the corresponding free energies $F_{\beta, \phi}$ converge, as $\beta \rightarrow \infty$, to $E_{\phi}$ in the sense of Gamma-convergence. As a consequence, the minimizer $\mu_{\beta, \phi}$ of $F_{\beta, \phi}$ converges in energy towards the weighted equilibrium measure $\mu_{\left(S_{0}, \phi\right)}$ of $S_{0}$.

Proof. Since $D_{\mu_{0}} \geq 0$ the lower bound in the Gamma-convergence follows directly from the lower semi-continuity of $E_{\phi}$ in the previous lemma. The reconstruction property then follows from the reconstruction property in the case when $\phi=0$ using the continuity statement in Lemma 3.12. That is to say that any reconstruction sequence for $E(\mu)$ is also a reconstruction sequence for $E_{\phi}(\mu)$.

We thus arrive at the following constructive version of the approximation in Theorem 1.1:

Theorem 3.15. Let $\mu_{0}$ be a measure in $\mathbb{R}^{d}$ with compact support $S_{0}$ such that $\mu_{0}$ does not charge polar sets and $\mu_{0}$ is determining. Given $\mu \in \mathcal{P}\left(S_{0}\right)$ such that $E(\mu)<\infty$, let $\mu_{\beta}$ be the minimizer of the free energy functional $F_{\beta, \psi_{\mu}}$ on $\mathcal{P}\left(S_{0}\right)$, i.e. $F_{\beta, \psi_{\mu}}:=E_{\psi}+\beta^{-1} D_{\mu_{0}}$. Then $\mu_{\beta}$ converges in energy towards $\mu$, as $\beta \rightarrow \infty$.

Proof. By definition $\mu=\Delta \psi_{\mu}$ and $\mu$ minimizes $E_{\psi_{\mu}}$ on $\mathcal{P}\left(S_{0}\right)$. Hence, the convergence follows directly from the convergence in the previous proposition.

This is a constructive approximation in the sense that there are quasi-explicit ways of approximating the minimizer $\mu_{\beta, \psi}$ of $F_{\beta, \psi}$, for a given $\psi \in \mathcal{E}_{S_{0}}\left(\mathbb{R}^{d}\right)$. For example, when $\psi \in \mathcal{E}_{S_{0}}\left(\mathbb{R}^{d}\right)$ is assumed bounded on $S_{0}$ it follows from Cor 4.9 below that the measure $\mu_{N, \beta}$ defined as the expectations $\mathbb{E}\left(\delta_{N}\right)$ of the empirical measure of the Riesz gas associated to the finite measure $e^{-\beta \psi} \mu_{0}$, converges in energy towards $\mu_{\beta}$ :

$$
\mu_{N, \beta}:=\mathbb{E}\left(\delta_{N}\right)=\frac{\int_{\left(\mathbb{R}^{d}\right)^{N-1}} e^{-\beta H\left(x, x_{2}, \ldots x_{N}\right)}\left(e^{-\beta \psi} \mu_{0}\right)^{\otimes N-1}}{\int_{\left(\mathbb{R}^{d}\right)^{N}} e^{-\beta H\left(x_{1}, \ldots x_{N}\right)}\left(e^{-\beta \psi} \mu_{0}\right)^{\otimes N}} e^{-\beta \psi(x)} \mu_{0},
$$


Priors leading to well-behaved Coulomb, Riesz gases vs zeroth-order phase transitions

where $H$ denotes the Hamiltonian of the Riesz gas. In the general case we can simply replace $\psi$ with $\max \{\psi,-R\}$ for a given parameter $R>0$ and obtain $E\left(\mu_{\beta}\right)$ from the double limit where first $N \rightarrow \infty$ and then $R \rightarrow \infty$. By a diagonal argument this yields a sequence of measures $\mu_{N, R_{N}}$, absolutely continuous with respect to $\mu_{0}$ and converging in energy towards a given measure $\mu \in \mathcal{P}\left(S_{0}\right)$ of finite energy. This is a quasi-explicit approximation in the sense that $\mu_{N, \beta}$ is given by a quotient of two integrals, whose integrands are explicitly given.

\subsection{The limit $\beta \rightarrow 0$}

Before turning to large deviations we note that in the opposite (infinite temperature) limit $\beta \rightarrow 0$ the minimizers $\mu_{\beta}$ always converge towards the reference measure $\mu_{0}$.

Proposition 3.16. Let $\mu_{0}$ be a measure on $\mathbb{R}^{d}$ which does not charge polar subsets and of compact support $S_{0}$ and assume that $\phi \in \mathcal{C}\left(S_{0}\right)$. Then $\beta F_{\beta, \phi} \rightarrow D_{\mu_{0}}$ as $\beta \rightarrow 0$ in the sense of Gamma-convergence on $\mathcal{P}\left(S_{0}\right)$. In particular, the minimizer $\mu_{\beta}$ of $F_{\beta}$ converges weakly towards $\mu_{0}$.

Proof. First observe that, since $D_{\mu_{0}} \leq \beta F_{\beta}:=D_{\mu_{0}}+\beta E_{\phi}$ and $D_{\mu_{0}}$ is lsc, the lower bound in the definition of Gamma-convergence is satisfied. All that remains is thus to show that for any $\mu \in \mathcal{P}\left(S_{0}\right)$ such that $D_{\mu_{0}}(\mu)<\infty$ there exists a recovery family $\mu_{\beta}$. In the case when $E(\mu)<\infty$ we can trivially take $\mu_{\beta}=\mu$. Next, note that there exists a sequence $\nu_{j} \in \mathcal{P}\left(S_{0}\right)$ such that $E\left(\nu_{j}\right)<\infty$ and $D_{\mu_{0}}\left(\nu_{j}\right) \rightarrow D_{\mu_{0}}\left(\mu_{0}\right)$ in $\mathcal{P}\left(S_{0}\right)$, as $j \rightarrow \infty$. Indeed applying Lemma 3.5 to $\phi=0$ and a fixed $\beta$, say $\beta=1$, shows that there exists a measure $\nu$ of finite energy which is absolutely continuous wrt $\mu_{0}$ :

$$
\nu=\rho \mu_{0}, \quad \rho \in L^{1}\left(\mu_{0}\right)
$$

Hence, the truncated sequence $\nu_{j}:=\max (\rho, j) / \int \nu_{j}$ has the required properties. Using a truncation argument again and the monotone convergence theorem then shows that any $\mu$ such that $D_{\mu_{0}}(\mu)<\infty$ has the property that there exists a sequence $\mu_{j} \in \mathcal{P}\left(S_{0}\right)$ such that $E\left(\mu_{j}\right)<\infty$ and $D_{\mu_{0}}\left(\mu_{j}\right) \rightarrow D_{\mu_{0}}(\mu)$. But then the recovery property for any $\mu$ follows by a simple diagonal argument.

\section{Large deviations}

We start with the following general setup. Let $X$ be a compact topological space and $W$ a symmetric proper lsc function on $X \times X$ called the pair interaction potential. Given a probability measure $\mu_{0}$ with support $X$ the corresponding Gibbs measures at inverse temperature $\left.\beta_{N} \in\right] 0, \infty[$ are defined as the following sequence of symmetric probability measures on $X^{N}$ :

$$
\mu_{\beta_{N}}^{(N)}:=\frac{1}{Z_{N, \beta_{N}}} e^{-\beta_{N} H^{(N)}} \mu_{0}^{\otimes N},
$$

where

$$
H^{(N)}\left(x_{1}, \ldots x_{N}\right):=\frac{1}{(N-1)} \frac{1}{2} \sum_{i \neq j} W\left(x_{i}, x_{j}\right)
$$

and the normalization constant $Z_{N, \beta_{N}}$ is assumed to be non-zero (it is automatically finite, since $W$ is lsc and $X$ is compact). We also assume that the following limit exists:

$$
\left.\left.\beta:=\lim _{N \rightarrow \infty} \beta_{N} \in\right] 0, \infty\right]
$$

Setting

$$
E(\mu):=\frac{1}{2} \int_{X^{2}} W \mu^{\otimes 2}
$$


Priors leading to well-behaved Coulomb, Riesz gases vs zeroth-order phase transitions

the corresponding free energy functional $F_{\beta}$ on $\mathcal{P}(X)$ is defined as in formula (3.1) (with $\phi=0$ ). The empirical measure $\delta_{N}$ (formula (1.4)) defines a $\mathcal{P}(X)$-valued random variable on $\left(X^{N}, \mu_{\beta_{N}}^{(N)}\right)$. By definition, its law is the probability measure

$$
\Gamma_{N}:=\left(\delta_{N}\right)_{*} \mu_{\beta_{N}}^{(N)}
$$

on $\mathcal{P}(X)$.

We recall the general definition of a Large Deviation Principle (LDP) for a sequence of measures [25], which is modeled on the classical Laplace steepest descent principle for integrals:

Definition 4.1. Let $Y$ be a compact Polish space, i.e. a compact complete separable metric space.

(i) A function $I: Y \rightarrow]-\infty, \infty]$ is a rate function if it is lower semi-continuous and $\inf _{Y} I=0$

(ii) A sequence $\Gamma_{N}$ of measures on $Y$ satisfies a large deviation principle with speed $r_{N}$ and rate function $I$ if

$$
\limsup _{N \rightarrow \infty} \frac{1}{r_{N}} \log \Gamma_{N}(\mathcal{F}) \leq-\inf _{\mu \in \mathcal{F}} I(\mu)
$$

for any closed subset $\mathcal{F}$ of $Y$ and

$$
\liminf _{N \rightarrow \infty} \frac{1}{r_{N}} \log \Gamma_{N}(\mathcal{G}) \geq-\inf _{\mu \in \mathcal{G}} I(\mu)
$$

for any open subset $\mathcal{G}$ of $Y$.

Fixing a metric on $Y$ The LDP may also be equivalently expressed in terms of $\Gamma_{N}\left(B_{\epsilon}(\mu)\right)$, where $B_{\epsilon}(\mu)$ denotes the closed ball of radius $\epsilon$ centered at $\mu \in Y$. For example, if $\Gamma_{N}$ is the law of the empirical measure $\delta_{N}$ of a random point process, then the LDP is equivalent [25, Theorems 4.1.11, 4.1.18 ] to

$$
\begin{aligned}
\lim _{\epsilon \rightarrow 0} \liminf _{N \rightarrow \infty} \frac{1}{r_{N}} \log \mathbb{P}\left(\frac{1}{N} \sum_{i=1}^{N} \delta_{x_{i}} \in B_{\epsilon}(\mu)\right) & =\lim _{\epsilon \rightarrow 0} \limsup _{N \rightarrow \infty} \frac{1}{r_{N}} \log \mathbb{P}\left(\frac{1}{N} \sum_{i=1}^{N} \delta_{x_{i}} \in B_{\epsilon}(\mu)\right) \\
& =-I(\mu)
\end{aligned}
$$

for some functional $I(\mu)$ (which, as a consequence, thus has to be lower semi-continuous).

Given $W$ and $\mu_{0}$ and a sequence $\beta_{N}$ as above we will say that the corresponding LDP holds at inverse temperature $\beta$ if the Gibbs measures $\mu_{\beta_{N}}^{(N)}$ are well-defined and the laws $\Gamma_{N}$ of the corresponding empirical measures on $X^{N}$ satisfy a LDP.

Theorem 4.2. Assume given a proper lsc pair interaction potential $W$ and a measure $\mu_{0}$ with compact support $X$.

- When $\beta \in] 0, \infty\left[\right.$ the corresponding LDP holds with speed $\beta N$ iff the functional $F_{\beta}$ is proper lsc on $\mathcal{P}\left(S_{0}\right)$ iff there exists a measure of finite energy, which is absolutely continuous wrt $\mu_{0}$. Then the rate functional is given by $F_{\beta}-\inf _{\mathcal{P}(X)} F_{\beta}$.

- When $\beta=\infty$ the corresponding LDP holds with speed $N \beta_{N}$ if $F_{\beta}$ is proper lsc for all $\beta \in] 0, \infty]$ and Gamma-continuous as $\beta \rightarrow \infty$. The rate functional is then given by $E-\inf _{\mathcal{P}(X)} E$.

Proof. This result is essentially contained in [29, 7, 32]. But for completeness we provide some details. First observe that since $X$ is compact $F_{\beta}$ is proper lsc iff $\inf _{\mathcal{P}\left(S_{0}\right)} F_{\beta}<\infty$. Since $E$ is bounded from below on $\mathcal{P}\left(S_{0}\right)$, the latter condition immediately implies 
Priors leading to well-behaved Coulomb, Riesz gases vs zeroth-order phase transitions

the existence of a measure $\mu$ of finite energy and which is absolutely continuous wrt $\mu_{0}$. Conversely, if such a measure $\mu$ exists then writing $\mu=\rho \mu_{0}$ and setting $\nu:=$ $\max \{1, \rho\} \mu_{0} / C$, where $C$ ensures that $\nu \in \mathcal{P}(K)$ gives $F_{\beta}(\nu)<\infty$. Indeed, $D_{\mu_{0}}(\nu)<\infty$ and $E(\nu)<\infty$, using that $W$ is bounded from below on $S_{0} \times S_{0}$.

Next, if $\inf _{\mathcal{P}\left(S_{0}\right)} F_{\beta}<\infty$ then the LDP for $\beta<\infty$ essentially follows from the results in $[29,7,32]$ (the converse is trivial since the rate functional of an LDP is proper lsc). For completeness let us recall the argument given in [7], which builds on the variational approach introduced in [47] (see also [40, 23] for similar results). Fix a continuous functional $\Phi$ on $\mathcal{P}\left(S_{0}\right)$ and set $H_{\Phi}^{(N)}:=H^{(N)}+N \delta_{N}^{*} \Phi, F_{\beta, \Phi}:=F_{\beta}+\Phi$ and

$$
\mathcal{F}_{\beta_{N}}^{(N)}[\Phi]:=-\frac{1}{N \beta_{N}} \log \int e^{-\beta_{N} H_{\Phi}^{(N)}} \mu_{0}^{\otimes N}
$$

Using Bryc's criterion for a LDP it is, as explained in [7], enough to prove that

$$
\lim _{N \rightarrow \infty} \mathcal{F}_{\beta_{N}}^{(N)}[\Phi]=\inf _{\mathcal{P}\left(S_{0}\right)} F_{\beta, \Phi}
$$

Note if $F_{\beta}$ is proper lsc, then so is $F_{\beta, \Phi}$. The starting point of the proof of the asymptotics (4.3) is Gibbs variational principle (which follows from Jensen's inequality):

$$
\mathcal{F}_{\beta_{N}}^{(N)}[\Phi]=N^{-1} \inf _{\mu_{N}}\left(\int_{X^{N}} H_{\Phi}^{(N)} \mu_{N}+D_{\mu_{0}^{\otimes N}}\left(\mu_{N}\right)\right)
$$

It implies, using that $W$ is lsc (to handle the energy term) and the sub-additivity of the entropy (see [7]), the lower bound

$$
\inf _{\mathcal{P}\left(S_{0}\right)} F_{\beta, \Phi} \leq \liminf _{N \rightarrow \infty} \mathcal{F}_{\beta_{N}}^{(N)}[\Phi]
$$

As for the corresponding upper bound

$$
\limsup _{N \rightarrow \infty} \mathcal{F}_{\beta_{N}}^{(N)}[\Phi] \leq \inf _{\mathcal{P}\left(S_{0}\right)} F_{\beta, \Phi}
$$

it is shown by taking $\mu_{N}=\mu^{\otimes N}$ in the rhs of formula (4.4), where $\mu$ realizes the infimum of the proper lsc functional $F_{\beta, \Phi}$ using that

$$
N^{-1} \int_{X^{N}} H^{(N)} \mu^{\otimes N}+\beta^{-1} N^{-1} D_{\mu_{0}^{\otimes N}}\left(\mu^{\otimes N}\right)=E(\mu)+\beta^{-1} D_{\mu}(\mu),
$$

if $E(\mu)<\infty$ (by the Fubini-Tonelli theorem) together with the basic fact $\left(\delta_{N}\right)\left(\mu^{\otimes N}\right) \rightarrow \delta_{\mu}$ weakly on $\mathcal{P}(X)$ to handle the term depending on $\Phi$.

Next consider the case when $\beta=\infty$. As pointed out above, in order to establish the LDP in question, it is enough to show that the limit (4.3) also holds for $\beta=\infty$. To this end first observe that the corresponding lower bound is easier since the entropy term can be dropped. Moreover, to prove the corresponding upper bound fix $\beta>0$ and note that, by Hölder's inequality,

$$
\mathcal{F}_{\beta_{N}}^{(N)}[\Phi] \leq \mathcal{F}_{\beta}^{(N)}[\Phi]
$$

for $N$ sufficiently large. Hence, the upper bound (4.6) for $\beta=\infty$ is obtained by first letting $N \rightarrow \infty$, then using the corresponding upper bound for $\beta<\infty$ and finally letting $\beta \rightarrow \infty$ and using the assumed Gamma-convergence of $F_{\beta}$ towards $F_{\infty}$.

Example 4.3. Even if $W$ is assumed bounded, the LDP may hold at $\beta=\infty$ with a rate functional which is different than $E-\inf E$. A simple such example is obtained by taking $X=[0,1], \mu_{0}=d x$ and $W(x, y):=V(x)+V(y)$ where $V$ is the proper lsc function 
Priors leading to well-behaved Coulomb, Riesz gases vs zeroth-order phase transitions

defined by $V(x)=0$ for $x \neq 0$ and $V(0)=-1$, say. Since $V=0$ a.e. wrt $d x$ we have that $\mu_{\beta}^{(N)}=d x^{\otimes N}$. But if the LDP would hold with a rate functional $E-\inf E$, then

$$
\lim _{N \rightarrow \infty}-\frac{1}{N \beta_{N}} \log Z_{N}=\inf _{\mathcal{P}([0,1])} E=\inf _{\mathcal{P}([0,1])} \int_{[0,1]} V \mu=\inf _{[0,1]} V=-1,
$$

which contradicts $Z_{N}=\int_{[0,1]} d x=1$. This example also illustrates that the expectations of the empirical measure $\delta_{N}$ (which here equals $d x$ ) may, in general, not converge to a minimizer of $E$ (which here equals $\delta_{0}$ ). Also note that in this example, the measure $\delta_{0}$ does not have the Energy Approximation property (since $E\left(\delta_{0}\right)=-1$, while $E(\mu)=0$ if $\mu=\rho d x$ ). Similarly, $F_{\beta}$ Gamma-converges to the constant functional 0. Moreover, in this setting the measure $d x$ is not determining, since setting $\psi:=\psi_{\delta_{0}}-1$ gives $\psi=-V$ and hence $\psi=0$ a.e. $d x$, while $\psi(0)>0$. Moreover, the Bernstein-Markov inequality fails in this example (with $\phi=0$ ), as is seen by taking $\mu=\delta_{0}$.

Modifying the previous example we also have the following example involving the Coulomb gas in the plane $\mathbb{C}$ subject to an exterior potential $\phi$, showing that the corresponding LDP does not always hold at $\beta=\infty$ if $\phi$ is lsc, but not continuous.

Example 4.4. Set $W(z, w):=-\log |z-w|^{2}+\phi(z)+\phi(w)$ for a given function $\phi$ in $\mathbb{C}$ and denote by $H_{\phi}^{(N)}$ and $E_{\phi}$ the corresponding $N$-particle Hamiltonian and energy functional, respectively. Consider the corresponding Gibbs measure at $\beta_{N}=N$, say, with $\mu_{0}$ given by Lebesgue measure on a given compact subset $X$ in $\mathbb{C}$. Fix a continuous function $\phi_{0}$ on $X$ and $t \geq 0$ and set $\phi:=\phi_{0}(z)-t \chi_{S^{1}}(z)$ in $\mathbb{C}$, where $\chi=1$ on the unit-circle $S^{1}$ and $\chi=0$ on the complement of $S^{1}$. Assume to get a contradiction that

$$
\liminf _{N \rightarrow \infty} N^{-2} \log Z_{N}[\phi] \geq-\inf _{\mathcal{P}(X)} E_{\phi}, \quad Z_{N}[\phi]:=\frac{1}{Z_{N, \beta_{N}}} \int e^{-\beta_{N} H^{(N)}} \mu_{0}^{\otimes N}
$$

Now, since the measure $\nu$ defined by the uniform measure on $S^{1}$ is a candidate for the inf of $E_{\phi}$, the rhs in formula (4.7) is bounded from below by $-E_{0}(\nu)-\phi_{0}(r)+t$ and $E_{0}(\nu)$ is finite. But $\phi_{t}=\phi_{0}$ a.e. wrt $\mu_{0}$ and hence $Z_{N}[\phi]=Z_{N}\left[\phi_{0}\right]$, which implies that the lhs in formula (4.7) is uniformly bounded from above by a finite constant $C_{0}$. Taking $t$ sufficiently large thus gives the desired contradiction. Finally, note that the same example applies in the non-compact case where $X=\mathbb{C}$ if $\phi_{0}(z) \geq(1+\epsilon) \log \left(\left.|1+| z\right|^{2}\right)-C$ for some $\epsilon>0$ and $C>0$.

As we will show in Section 4.2 the Gamma-continuity assumption on $F_{\beta}$ is not necessary for the existence of a LDP at $\beta=\infty$ with rate functional $F_{\beta}-\inf F_{\beta}$. On the other hand, by the previous example it it is not enough to assume that $F_{\infty}$ is proper lsc. In the case of the $2 \mathrm{~d}$ Coulomb gas, this will be illustrated using the well-known notion of Bernstein-Markov inequalities. This notion can be extended to a general pair interaction potential $W$ as follows (the case of the Riesz gas was introduced in [18]).

Definition 4.5. Given $\phi \in \mathcal{C}(X)$ we will say that a measure $\mu_{0}$ satisfies the weighted Bernstein-Markov inequality (wrt the pair interaction $W$ ) if for any $\epsilon>0$ there exists a constant $C>0$ such that for any $p>0$

$$
\sup _{X} e^{\psi_{\mu}-\phi} \leq C^{1 / p} e^{\epsilon}\left\|e^{\psi_{\mu}-\phi}\right\|_{L^{p}\left(S_{0}, \mu_{0}\right)}
$$

for all discrete measures $\mu$ of the form $\mu=N^{-1} \sum_{i=1}^{N} \delta_{x_{i}}$, for some $x_{i} \in X$. We say that $\mu_{0}$ satisfies the strong Bernstein-Markov property if it satisfies the weighted BernsteinMarkov inequality for all $\phi \in \mathcal{C}(X)$. 
Priors leading to well-behaved Coulomb, Riesz gases vs zeroth-order phase transitions

The following result shows, in particular, that the Bernstein-Markov inequality is a sufficient condition for the LDP to hold at zero-temperature if $E$ is proper lsc and strictly convex (see [5, 16] for the logarithmic case and complex case and [18] for the case of the Riesz gas).

Theorem 4.6. Assume that $E$ is proper lsc on $\mathcal{P}(X)$.

- If $\mu_{0}$ has support $X$ and satisfies the Bernstein-Markov inequality, then

$$
-\lim _{N \rightarrow \infty} \frac{1}{N \beta_{N}} \log Z_{N}=\inf _{\mathcal{P}(X)} E
$$

and the following concentration property holds: any limit point $\Gamma$ in $\mathcal{P}(\mathcal{P}(X))$ of the law $\Gamma_{N}$ of the empirical measure $\delta_{N}$ is supported in $\arg \inf _{\mathcal{P}(X)} E$. In particular, if $E$ admits a unique minimizer $\mu$, then $\delta_{N}$ converges in law towards $\mu$.

- If $\mu_{0}$ has the strong Bernstein-Markov property and $E$ is strictly convex on $\mathcal{P}(X)$, then the $L D P$ holds at a speed $\beta_{N} N$ and with rate functional $E-\inf _{\mathcal{P}(X)} E$.

Proof. Set $\Phi(\mu)=\langle\mu, \phi\rangle$ for a given $\phi \in C(X)$ and assume that $\mu_{0}$ satisfies the weighted Bernstein-Markov-inequality for the weight $\phi$. Then,

$$
\limsup _{N \rightarrow \infty} \mathcal{F}_{\beta_{N}}^{(N)}[\Phi] \leq \inf _{\mathcal{P}(X)}(E+\Phi)
$$

To see this, first observe that the function $\psi$ on $X$ obtained by freezing all but one of arguments in $H^{(N)}\left(x_{1}, x_{2}, \ldots, x_{N}\right)$ is of the form $\psi_{\mu}$ for $\mu$ a discrete measure of the form appearing in the definition of the Bernstein-Markov-inequality. Hence, using the weighted Bernstein-Markov-inequality $N$ times gives

$$
\limsup _{N \rightarrow \infty} \mathcal{F}_{\beta_{N}}^{(N)}[\Phi] \leq \limsup _{N \rightarrow \infty} N^{-1} \inf _{X^{N}} H_{\Phi}^{(N)}
$$

The bound (4.10) now follows from the following fact, which holds for any $\Phi \in C(\mathcal{P}(X))$ :

$$
\lim _{N \rightarrow \infty} N^{-1} \inf _{X^{N}} H_{\Phi}^{(N)}=\inf _{\mathcal{P}(X)}(E+\Phi)
$$

This is essentially well-known and classical (a proof is provided below). Now, combining the upper bound (4.10) with the corresponding lower bound (4.5) (which always holds) gives

$$
\lim _{N \rightarrow \infty} \mathcal{F}_{\beta_{N}}^{(N)}[\Phi]=\inf _{\mathcal{P}(X)}(E+\Phi)
$$

for all linear and continuous $\Phi$. In particular, specializing to $\Phi=0$ proves (4.9). To prove the concentration property in the first point we note that the lower bound (4.5) can be refined to give

$$
\int_{\mathcal{P}(X)} E \Gamma \leq \liminf _{N \rightarrow \infty} \mathcal{F}_{\beta_{N}}^{(N)}[0]
$$

Combining this inequality with (4.12) (for $\Phi=0$ ) and using that $E$ is lsc gives the concentration property in question.

Finally, if the Bernstein-Markov-property holds for all $\phi$, then the asymptotics (4.12) hold for all linear bounded functionals $\Phi$. Hence, if $E$ is strictly convex the LDP in question follows from the Gärtner-Ellis theorem (see [6, Lemma 4.7] for a convenient reformulation of the Gärtner-Ellis theorem).

Proof of the asymptotics (4.11): 
Priors leading to well-behaved Coulomb, Riesz gases vs zeroth-order phase transitions

We follow the argument in the proof of Theorem 4.2. By (4.5) it is enough to prove the corresponding upper bound. To this end fix $\beta>0$ and note that, since, $\inf _{X^{N}} H_{\Phi}^{(N)}$ is trivially bounded from above by $\mathcal{F}_{\beta}^{(N)}[\Phi]$ the upper bound (4.6) gives, for a fixed $\mu_{0}$ on $X$,

$$
\limsup _{N \rightarrow \infty} N^{-1} \inf _{X^{N}} H_{\Phi}^{(N)} \leq \inf _{\mathcal{P}(X)}\left(E_{\Phi}+\beta^{-1} D_{\mu_{0}}\right)
$$

Thus, the upper bound in question is obtained by taking $\mu_{0}$ as the minimizer of $E_{\Phi}$ (using that $\left.D_{\mu_{0}}\left(\mu_{0}\right)=0\right)$. We note that this proof of (4.11) is closely related to the proof of the result in [7, Cor 1.6], saying that $N^{-1} H^{(N)}$, identified with a functional on $\mathcal{P}(X)$, Gamma-converges towards $E$ (which implies (4.11) and is, in fact, equivalent to (4.11) for all $\Phi)$.

Remark 4.7. The proof of the first point is similar to the proof of the corresponding result in [41], which is claimed without any assumptions on $\mu_{0}$ (see the discussion in Section 1.4). The main difference is that the Bernstein-Markov-property of $\mu_{0}$ is used here to justify the upper bound in [41, Lemma 4], which does not hold for a general $\mu_{0}$ (by Example 4.3) and which corresponds to (4.10) here. See also [18, Section 4] for another approach based on the Bernstein-Markov-property.

\subsection{The case of the Riesz gas}

Let us now specialize to the case of the Riesz gas, i.e. the case when the pair interaction potential $W(x, y)$ is taken as the Riesz kernel $W_{\alpha}$ (section 2). The following result contains, in particular, the LDP for the Coulomb gas $(\alpha=2)$ appearing in Theorem 1.2 in the introduction.

Theorem 4.8. Assume that $\alpha \in] 0, d\left[\right.$. Given a measure $\mu_{0}$ with compact support $S_{0}$, not charging polar subsets, the following holds for the corresponding Riesz gas:

- For any $\beta \in] 0, \infty\left[\right.$, the $L D P$ holds with speed $\beta N$ and rate functional $F_{\beta}-\inf _{\mathcal{P}(X)} F_{\beta}$.

- If $\alpha \leq 2$, then the LDP holds for $\beta \in] 0, \infty]$ at a speed $\beta_{N} N$ with a rate functional which is continuous wrt Gamma-convergence iff $\mu_{0}$ is strongly determining.

Proof. Combining Theorems 3.11, 4.2 we just have to verify that if $\mu_{0}$ does not charge polar subsets, then the assumption in the first point of Theorem 4.2 is satisfied. But this follows from Lemma 3.5, by taking $\mu=\mu_{\beta}$.

Corollary 4.9. Assume that $\alpha \in] 0, d\left[\right.$ and let $\mu_{0}$ be as in the previous theorem and denote by $\mu_{N, \beta}$ the expectation of the empirical measure of the corresponding Riesz gas. In other words, $\mu_{N, \beta}$ is the push-forward to $\mathbb{R}^{d}$ of the Gibbs measure defining the Riesz gas. Then $\mu_{N, \beta}$ converges in energy towards the minimizer $\mu_{\beta}$ of the free energy functional $F_{\beta}$.

Proof. The weak convergence of $\mu_{N, \beta}$ towards $\mu_{\beta}$ follows directly form the LDP in the previous theorem. To prove that $E\left(\mu_{N, \beta}\right) \rightarrow E\left(\mu_{\beta}\right)$ it is, by basic integration theory, enough to show that there exists a constant $C_{\beta}$ such that

$$
\mu_{N, \beta} \leq C_{\beta} \mu_{0}
$$

To prove this inequality first observe that

$$
\frac{\mu_{N, \beta}}{\mu_{0}} \leq\left\{\sup _{S_{0}} e^{\beta \psi}: \psi \in \mathcal{L}_{S_{0}}(\mathbb{R}): \int e^{\beta \psi} \mu_{0}=1\right\}
$$

using that $H\left(x, x_{2}, \ldots x_{N}\right)$ is in $\mathcal{L}_{S_{0}}(\mathbb{R})$ for any fixed $\left(x_{2}, \ldots, x_{N}\right)$ and integrating over $S_{0}^{N-1}$. By Prop 3.6 thus shows that, for any given $\epsilon>0$ there exists a constant $C_{\epsilon}$ such that $\mu_{N, \beta} \leq C_{\epsilon} e^{\epsilon \beta} \mu_{0}$ which, in particular, implies the inequality (4.13). 
Remark 4.10. It follows from the LDP above, when $\alpha \leq 2$ (and its proof), that the functional $\mathcal{F}_{(S, \phi)}$ defined by formula (2.17) can be expressed in terms of the moment generating function of the corresponding empirical measure:

$$
\mathcal{F}_{\left(S_{0}, \phi\right)}(u)=\lim _{N \rightarrow \infty} \frac{1}{\beta_{N} N} \log \mathbb{E}\left(e^{N \beta_{N} \sum_{i=1}^{N} u\left(x_{i}\right)}\right)
$$

In the complex-geometric setting in [5] (which covers in particular the case when $d=\alpha=2$ ) the proof of the corresponding LDP goes the other way around: first the analog of (4.14) is established and then the LDP is deduced from the Gärtner-Ellis theorem.

According to Theorem 4.6 the Bernstein-Markov-property of a measure $\mu_{0}$ is a sufficient criterion for the LDP to hold at $\beta=\infty$. However, in general, the corresponding rate functional is not Gamma-continuous up to $\beta=\infty$, even if $\mu_{0}$ is assumed to be absolutely continuous wrt $d x$. This will be exemplified in the following section.

\subsection{The 2d Coulomb gas and orthogonal polynomials on the real line}

Now consider the "logarithmic case" $\alpha=2=d$, i.e. the Coulomb gas on a measure $\mu_{0}$ in $\mathbb{R}^{2}$ that we shall identify with $\mathbb{C}$. In this section we assume that the support $S_{0}$ of $\mu_{0}$ is contained in $\mathbb{R} \subset \mathbb{C}$.

Lemma 4.11. Assume that $\mu_{0}$ has compact support $S_{0}$ contained in $\mathbb{R}$. Then it has the Bernstein-Markov inequality iff it satisfies the weighted Bernstein-Markov inequality for all weights $\phi$ (i.e. iff it has the strong Bernstein-Markov property). Similarly, $\mu_{0}$ is determining iff it is strongly determining.

Proof. This is well-known, but for completeness we recall the argument. First assume that the Bernstein-Markov inequality holds in the non-weighted case, $\phi=0$. Now take a general continuous function $\phi$ on $\mathbb{R}$. In a neighborhood of $S_{0}$ we can express $-\phi$ as the uniform limit of $\log \left(\left|q_{k}\right|^{2}\right)$ for some polynomials $q_{k}$ on $\mathbb{C}$ of degree $k$ (using the Stone-Weierstrass theorem). The Bernstein-Markov inequality wrt $\phi$ then follows from the non-weighted one by replacing $p_{k}$ in formula (1.11) with $p_{k} q_{k}$. Similarly, if $\mu_{0}$ is determining for $\left(S_{0}, 0\right)$, then it is also determining for $\left(S_{0}, \phi\right)$, as shown by replacing $\psi$ in formula (1.2) with $\psi+k^{-1} \log \left(\left|q_{k}\right|^{2}\right)$.

Combining Theorem 4.6 with Proposition 4.13 below now gives the following characterization of measures $\mu_{0}$ on $\mathbb{R}$ such that the corresponding LDP holds at $T=0$ :

Theorem 4.12. Let $\mu_{0}$ be a measure whose support is a compact regular subset $S_{0}$ of $\mathbb{R}$ and such that $\mu_{0}$ does not charge polar subsets. Then the LDP for the corresponding Coulomb gas at $T=0$ holds with rate functional $E-\inf E$ iff $\mu_{0}$ satisfies the BernsteinMarkov-inequality. As a consequence, it is not enough to assume that $E$ is proper lsc (i.e. that $S_{0}$ is non-polar) for the LDP to hold at $T=0$. More precisely, there exists a measure $\mu_{0}$ with support $[-1,1]$, which is absolutely continuous wrt $d x$ and such that the corresponding expectations $\mathbb{E}\left(\delta_{N_{k}}\right)$ do not converge towards the equilibrium measure of $[-1,1]$ when $\beta_{N}=N-1$ and $N \rightarrow \infty$.

Proof. To prove the "only if" direction we set $\beta_{N}=N-1$ (and hence $\beta=\infty$ ) and note that $p_{N}=2$ in formula (1.10). This means that the corresponding Coulomb gas in $\mathbb{C}$ defines a determinantal point process with correlation kernel $K_{k}(z, w)$, where $K_{k}$ is the integral kernel of the orthogonal projection from $L^{2}\left(\mathbb{C}, \mu_{0}\right)$ onto the space $\mathcal{P}_{k}(\mathbb{C})$ of all polynomials $p_{k}(z)$ on $\mathbb{C}$ of degree at most $k:=N-1$ :

$$
K_{k}(z, w)=\sum_{j=0}^{k} p_{j}(z) \overline{p_{j}(w)},
$$


for an orthonormal base $p_{j}$ in $\mathcal{P}_{k}(\mathbb{C})$ (known as the Christoffel-Darboux kernel in the literature on orthogonal polynomials and the Bergman kernel in the complex analysis literature). In fact, this is the case for any measure $\mu_{0}$ on $\mathbb{C}$ not charging polar subsets (see, for example, [9]). Accordingly, it follows from general properties of determinantal point processes that

$$
\mathbb{E}\left(\delta_{N_{k}}\right)=\frac{1}{k+1} K_{k}(x, x) \mu_{0}
$$

Now, if the LDP holds at $T=0$ with rate functional $E$, then it follows, in particular, that $\mathbb{E}\left(\delta_{N_{k}}\right)$ converges towards the equilibrium measure $\mu_{S_{0}}$. But combining formula (4.16) with Prop 4.13 below then implies that $\mu_{0}$ satisfies the Bernstein-Markov-inequality. For the last statement it is enough to construct a measure $\mu_{0}$ on $\mathbb{R}$ not charging polar subsets and not satisfying the BM-inequality. The existence of such a measure is without doubt well-known to experts, but for completeness a concrete such measure is constructed in the appendix.

The following proposition, used in the proof of the previous theorem, is an unpublished result of Totik (thanks to Norman Levenberg for pointing this out).

Proposition 4.13. Let $\mu_{0}$ be a measure whose support is a compact regular subset $S_{0}$ of $\mathbb{R}$ and such that $\mu_{0}$ does not charge polar subsets. Denote by $K_{k}$ the corresponding kernel defined by formula (4.15). If $\frac{1}{k+1} K_{k}(x, x) \mu_{0}$ converges weakly towards the equilibrium measure $\mu_{S_{0}}$, then $\mu_{0}$ satisfies the Bernstein-Markov inequality.

Proof. Let us explain how to deduce this from the results in [17] concerning measures $\mu_{0}$ with compact support $S_{0} \subset \mathbb{R}$. We denote by $p_{k}$ the sequence of orthonormal polynomials in $L^{2}\left(\mu_{0}\right)$ associated to $\mu_{0}$ of degree $k$, by $\gamma_{k}$ the positive non-vanishing leading coefficient of $p_{k}$, i.e. $p_{k}=\gamma_{k} x^{k}+O\left(x^{k-1}\right)$ and by $\nu_{k}$ the empirical measure on the zeroes of $p_{k}$. The proposition then follows directly from combining the following three results proved in [55, Thm 3.2.3], [53, Thm 13.1] and [55, Cor 2.2.3], respectively:

(1) If $S_{0}$ is regular, then $\mu_{0}$ satisfies the BM-inequality iff $\mu_{0}$ is regular in the sense of Saff-Totik i.e.

$$
\lim _{m \rightarrow \infty} m^{-1} \log \gamma_{m}=\inf _{S_{0}} E
$$

(the lower bound holds for any $\mu_{0}$ )

(2) $\frac{1}{k+1} K_{k}(x, x) \mu_{0}$ converges weakly towards $\mu \in \mathcal{P}(S)$ iff $\nu_{k}$ converges weakly towards $\mu \in \mathcal{P}(S)$

(3) If $S_{0}$ has non-zero capacity (i.e $\inf _{S_{0}} E$ is finite) and $\nu_{k}$ converges weakly towards the equilibrium measure $\mu_{S_{0}}$, then either $\mu_{0}$ is regular or there exists a polar Borel subset $C \subset S_{0}$ such that $\mu(C)=\mu_{0}\left(S_{0}\right)$.

The proof of the previous proposition relies on special properties of orthonormal polynomials on subsets of real line, not shared by general orthonormal polynomials on subsets of $\mathbb{C}$. Accordingly, the equivalence in Theorem 4.12 is widely open in the general logarithmic setting in $\mathbb{C}$ (as well as in higher dimensions). This being said, Theorem 1.2 can be viewed as a general variant of Theorem 4.12 where the property of being Bernstein-Markov property is replaced by the stronger property of being determining (and then the conclusion is also stronger). By Prop 3.6 this amounts to demanding that the Bernstein-Markov inequality (4.8) holds for all potentials $\psi_{\mu}$. 
Priors leading to well-behaved Coulomb, Riesz gases vs zeroth-order phase transitions

\subsection{Proof of Cor 1.4}

By Theorem 1.2 we just have to provide a measure $\mu_{0}$ with support $K \subset \mathbb{C}$, which is absolutely continuous wrt Lebesgue measure (and, hence does not charge polar subsets) with the BM-property, but which is not determining. When $K=[-1,1]$ such an example has been constructed by Totik (reported in [17]) and as indicated in [17], the general case is similar (for completeness a proof is provided in the appendix).

\section{Relations to the Ehrenfest classification of phase transitions}

\subsection{The general setting}

Let us start by recalling the classical Ehrenfest classification of phase transitions in a general statistical mechanical setting, where the Hamiltonian $H^{(N)}$ in formula (4.1) is replaced by a general measurable (not necessarily symmetric) function on $\left(X^{N}, \mu_{0}^{\otimes N}\right)$. The corresponding free energy at temperature $T_{N}$ is defined by

$$
F_{N, T_{N}}=-\frac{T_{N}}{N} \log \int_{X_{N}} e^{-\frac{1}{T_{N}} H^{(N)}} \mu_{0}^{\otimes N},
$$

assuming that it is finite. By definition, there is a phase transition of order $m$ at temperature $T \in] 0, \infty]$ if, for any sequence $T_{N} \rightarrow T$ the limit

$$
f(T):=\lim _{N \rightarrow \infty} F_{N, T_{N}}
$$

exists and the derivatives of order $j=1, \ldots, m$ exist at $T$, but not the derivative of order $m+1$. It should be stressed that the notion of a phase transitions is often used in a broader sense (as discussed in the case of the Coulomb case in [54]), but here we shall be concerned only with the Ehrenfest classification.

We recall that phase transitions have been studied extensively in the setting of spin models, such as the Ising and Potts models on graphs, where the space $X$ is finite. For example, on the complete graph with $N$ nodes the (ferromagnetic) Potts model is defined by the Hamiltonian $H^{(N)}$ of the form (4.1) with pair-interaction $W(x, y)=-x \cdot y$ and space $X=\{1,2, . . q\}$ for a given integer $q \geq 2$, endowed with the counting measure $\mu_{0}$. The case $q=2$ is the Ising model on the complete graph (known as the Curie-Weiss model for magnetism). As is well-known, there is a critical critical temperature $\left.T_{c} \in\right] 0, \infty[$ such that $f(T)$ is smooth for $T>T_{c}$ and a phase transition occurs at $T=T_{c}$, which is of order two when $q=2$ and order one when $q \geq 3$ [58]. Moreover, according to the "mean-field philosophy" this implies phase transitions for the Ising and Potts model on $\mathbb{Z}^{d}$, when $d$ is sufficiently large [14].

However, by the following basic lemma, there are no zeroth-order phase transitions when $T>0$, i.e. no points where $f$ is discontinuous:

Lemma 5.1. If the limit (5.1) exists for any $T \in] 0, \infty[$, then $f$ is concave and increasing on $] 0, \infty[$ and, in particular, continuous.

Proof. If the limits exists then we can take $T_{N}=T$ for all $T$ and observe that $T \mapsto F_{N, T}$ is concave and increasing (as follows, for example, from Gibbs variational principle (4.4)). Since these properties are preserved by point-wise convergence the lemma follows.

Moreover, the following lemma explains why zeroth-order phase transitions do not appear, even at $T=0$, in the spin models discussed above.

Lemma 5.2. For a Hamiltonian of the form (4.1), with lower semi-continuous pair interaction potential $W$, there is no zeroth-order phase transition under the following condition:

$$
\lim _{T \rightarrow 0} \inf _{\mathcal{P}(X)}\left(E+T D_{\mu_{0}}\right)=\inf _{\mathcal{P}(X)} E
$$


Priors leading to well-behaved Coulomb, Riesz gases vs zeroth-order phase transitions

In particular, this is the case if the pair interaction potential $W$ is continuous.

Proof. By (4.5) (for $\Phi=0$ )

$$
\inf _{\mathcal{P}(X)} E \leq f(0) .
$$

Since $f(0) \leq f(T)$ letting $T \rightarrow 0$ it follows from the assumption that $f$ is continuous at $T=0$, as desired. To prove the last statement note that, since $D$ is lsc we have, in general, that

$$
\lim _{T \rightarrow 0} \inf _{\mathcal{P}(X)}\left(E+T D_{\mu_{0}}\right)=\lim _{T \rightarrow 0}\left(E\left(\mu_{T}\right)+T D_{\mu_{0}}\left(\mu_{T}\right)\right) \leq \liminf _{T \rightarrow 0} E\left(\mu_{T}\right)
$$

But if $E$ is continuous, then it follows from the compactness of $\mathcal{P}(X)$ that the rhs above is equal to the infimum of $E$.

Finally, we make the following observation (which applies in particular to Riesz interactions when $\mu_{0}$ satisfies a Bernstein-Markov inequality):

Lemma 5.3. Assume that $e^{-H^{(N)}}$ is continuous on $X^{N}$ and that there exists a sequence $\epsilon_{N}$ in $\mathbb{R}$, tending to zero, such that

$$
F_{N, T_{N}} \leq \inf _{X^{N}} \frac{1}{N} H^{(N)}+\epsilon_{N}
$$

Then there is a zeroth-order phase transition at $T=0$ iff

$$
\lim _{T \rightarrow 0} \lim _{N \rightarrow \infty} F_{N, T} \neq \lim _{N \rightarrow \infty} \lim _{T \rightarrow 0} F_{N, T}
$$

Proof. By the continuity assumption $\lim _{T \rightarrow 0} F_{N, T}=\inf _{X^{N}} \frac{1}{N} H^{(N)}$. Indeed, in general, the $L^{p}\left(\mu_{0}\right)$-norms of a bounded function $f$ on a compact set $K$ converge, as $p \rightarrow \infty$, to the essential sup $\|f\|_{L^{\infty}\left(K, \mu_{0}\right)}$ (compare Step 3 in the proof of Prop 3.6). In the present case $f=e^{-H^{(N)}}$ is continuous and hence the essential sup coincides with the ordinary sup. Moreover, if the inequality in the lemma holds then necessarily $f(0)=\lim _{N \rightarrow \infty} \inf _{X^{N}} \frac{1}{N} H^{(N)}$. Hence, the rhs in (5.2) is equal to $f(0)$, while the lhs is equal to $\lim _{T \rightarrow 0} f(T)$.

While there is an abundance of first and second order phase transitions in the physics and mathematics literature, zeroth-order phase transition appear to be of a rather pathological nature. Still, there has been some speculations on zeroth-order phase transitions in the physics literature in the context of superfluidity (see [45]) and black holes [36]. To the best of the authors knowledge there are, however, no previous examples of zeroth-order phase transitions in the rigorous sense described above.

\subsection{Phase transitions for the 2d Coulomb gas}

Now consider the setting of the Coulomb gas in $\mathbb{R}^{2}$ with a given exterior continuous potential $\phi$ and fix a measure $\mu_{0}$ on $\mathbb{R}^{2}$ which has the Bernstein-Markov property. Then the corresponding free energy $f_{\phi}(T)$ exists for all $T \in[0, \infty[$ (by Theorem 4.6). Any measure $\mu_{0}$ as in Corollary 1.4 provides an example of such a measure, for which the corresponding Coulomb gas has a zeroth-order phase transition.

We recall that phase transitions are also frequently studied as the strength of $\phi$ is varied (in the standard case of spin systems $\phi(x):=-x$ ). This means that $\phi$ is replaced by

$$
\phi_{h}:=\phi_{0}+h \phi
$$


Priors leading to well-behaved Coulomb, Riesz gases vs zeroth-order phase transitions

for a given parameter $h \in \mathbb{R}$ and continuous functions $\phi_{0}$ and $\phi$. We then set

$$
f(T, h):=f_{\phi_{h}}(T)
$$

for $(T, h) \in[0, \infty[\times \mathbb{R}$. Set $T=0$ and consider the function $h \mapsto f(T, h)$. By Prop 2.10 there is no zeroth or first order phase transitions. A third order phase transition was discovered by Gross-Witten in the context of lattice gauge theories and unitary random matrices [34] (and used in [39] to study the expected length of the longest increasing subsequence in a random permutation). This phase transition concerns the case when $\mu_{0}$ is the invariant measure on the unit-circle $S^{1}$ in $\mathbb{C}, \phi_{0}=0$ and $\phi(z)$ is half the real part of $z$, i.e. equal to $\cos \theta$ on $S^{1}$ (the phase transition appears at $h=2$ ). See [44] or a general discussion about third-order phase transitions for $2 \mathrm{~d}$ Coulomb gases. Here we give simple examples of second order phase transitions on the unit-disc.

Proposition 5.4. Consider the Coulomb gas in $\mathbb{R}^{2}$ and let $\mu_{0}$ be normalized Lebesgue measure on the closed unit-disc $K$ and $\phi$ a non-constant radial subharmonic function $\phi$ on a neighborhood of $K$. Set $\phi_{h}=h \phi$. Then the corresponding function $h \mapsto f(0, h)$ is differentiable at $h=0$, but not two times differentiable.

Proof. In order to use standard complex analytic normalizations it will be convenient to use a normalization where $\Delta:=\frac{1}{4 \pi}\left(\partial_{x}^{2}+\partial_{y}^{2}\right)$. These normalizations ensure that $\Delta \log |z|^{2}$ is the uniform probability measure on $S^{1}$. First observe that without loss of generality we may, by replacing $\phi$ by $A \phi+B$ assume that $\int \Delta \phi \leq 1$ on $K$ and $\phi=0$ on $\partial K$. By the maximum principle it then follows that $\phi \leq 0$ in $K$. Set $\psi_{h}:=\log |z|^{2}$ when $|z| \geq 1$. For $|z| \leq 1$ we set $\psi_{h}=h \phi$ when $h \geq 0$ and $\psi_{h}=0$ if $h<0$ and make the following

$$
\text { Claim: } \psi_{h}=P_{K}(h \phi) \text {. }
$$

First observe that $\psi_{h}(z)$ is subharmonic. Indeed, writing $\psi_{h}(z)=\Phi(x)$ for $x:=\log |z|^{2}$ the subharmonicity of $\psi_{h}$ is equivalent to the convexity of $\Phi$, which in turn follows from noting that $\Phi(x)=x$ when $x \geq 0$ and when $x \leq 0$ we have $\partial_{x}^{2} \Phi \geq 0$ and

$$
\partial_{x} \Phi(0)=\int_{-\infty}^{0} \partial_{x}^{2} \Phi=\int_{K} \Delta \phi \leq 1
$$

Moreover, this implies that, when $h \geq 0$,

$$
\Delta \psi_{h}=(1-c h) \delta_{\partial K}+h \Delta \phi, \quad c=\int_{K} \Delta \phi\left(=1-\partial_{x} \Phi(0)\right)
$$

where $\delta_{\partial K}$ denotes the uniform measure on the unit-circle $\partial K$. Moreover, when $h<0$,

$$
\Delta \psi_{h}=\delta_{\partial K}
$$

Hence, $\psi_{h} \leq h \phi$ on $K$ and $\psi_{h}=h \phi$ almost everywhere with respect to $\Delta \psi_{h}$. The claim above thus follows from the domination principle. Now, by Prop 2.10 we have that

$$
\frac{d f(0, h)}{d h}=\int_{K} \phi \Delta \psi_{h}=\int_{|z|<1} \phi \Delta \psi_{h},
$$

using that $\phi=0$ on the boundary of the unit-disc $K$. By the previous discussion this means that $\frac{d f(0, h)}{d h}$ vanishes identically when $h<0$ and is equal to $h$ times the nonzero number $\int_{|z|<1} \phi \Delta \phi$ when $h \geq 0$. Hence, $\frac{d f(0, h)}{d h}$ is not differentiable at $h=0$, as desired. 
Priors leading to well-behaved Coulomb, Riesz gases vs zeroth-order phase transitions

Remark 5.5. The second order phase transition above can be contributed to the fact that the support of the weighted equilibrium measure $\mu_{h}$ changes drastically at $h=0$ : for $h>0$ it contains a disc inside $K$, which disappears when $h \leq 0$.

In particular, if $\phi=|z|^{2}$, say, and if $\mu_{0}$ is taken as the measure whose support is the unit-disc, provided by Corollary 1.4, then the corresponding Coulomb gas exhibits a rather peculiar phase diagram in the $(T, h)$-plane. Indeed, for any fixed $h$ there is a zeroth-order phase transition as $T \rightarrow 0^{+}$and moreover, when $T=0$ there is a second order phase transition as $h \rightarrow 0$. Let us also remark that, comparing with standard physics terminology, the measure

$$
\mu_{h, T}:=\lim _{N \rightarrow \infty} \mathbb{E}\left(\delta_{N}\right)
$$

(which minimizes the corresponding free energy functional) plays the role of an order parameter for the phase transitions (which usually appears as a physical observable). By Theorem 3.10, a zeroth-order phase transition at $T=0$ is equivalent to the discontinuity of $T \mapsto \mu_{T, 0}$, viewed as a curve in $\mathcal{P}\left(S_{0}\right)$. Equivalently, this means that there exists some (smooth) exterior potential $\phi$ such that the corresponding free energy $f(T, h)$ satisfies

$$
\lim _{T \rightarrow 0^{+}} \frac{\partial f(T, 0)}{\partial h} \neq \frac{\partial f(0,0)}{\partial h}
$$

\section{Appendix}

\subsection{Capacities and determining measures}

We start by recalling the notion of (non-weighted) capacity, mainly following [42]. Given a parameter $\alpha \in] 0, d\left[\right.$ the corresponding capacity of a compact set $K \subset \mathbb{R}^{d}$ is defined by

$$
\mathcal{C}_{\alpha}(K):=1 / \inf _{\mu \in \mathcal{P}(K)} E(\mu),
$$

where $E$ is the energy of $\mu$. The inner capacity of a general bounded set $S \subset \mathbb{R}^{d}$ is defined by

$$
\mathcal{C}_{\alpha}(S)_{*}=\sup _{K \subset S} \mathcal{C}_{\alpha}(K)
$$

where the sup ranges over all compact subsets $K$ of $S$. Similarly, the outer capacity is defined by

$$
\mathcal{C}_{\alpha}(S)^{*}:=\inf _{S \subset U} \mathcal{C}_{\alpha}(U)
$$

where the sup ranges over all bounded open sets $U$ containing $S$. A bounded subset $S$ is said to be polar if $\mathcal{C}_{\alpha}(S)^{*}=0$. This equivalently means that there exists a potential $\psi$ such that $S \Subset\{\psi=-\infty\}$. A subset $S$ is said to be capacitable if $\mathcal{C}_{\alpha}(S)_{*}=\mathcal{C}_{\alpha}(S)^{*}$. Any bounded Borel set $S$ is capacitable. The set functional $\mathcal{C}_{\alpha}$ is invariant under translations and satisfies Choquet's capacity axioms on Borel sets:

- (monotonicity) If $E \subset F$ then $\mathcal{C}_{\alpha}(E) \leq \mathcal{C}_{\alpha}(F)$.

- (inner continuity) If $S_{i}$ is a sequence of sets increasing to $S$ and $S=\bigcup_{i} S_{i}$, then $\mathcal{C}_{\alpha}\left(S_{i}\right) \rightarrow \mathcal{C}_{\alpha}(S)$

- (outer continuity). If $K_{i}$ is a sequence of compact sets decreasing to the compact set $K$, then $\mathcal{C}_{\alpha}\left(K_{i}\right) \rightarrow \mathcal{C}_{\alpha}(K)$

Moreover, $\mathcal{C}_{\alpha}$ is sub-additive: given a sequence of compact subset $K_{j}$

$$
\mathcal{C}_{\alpha}\left(\bigcup_{j} K_{j}\right) \leq \sum_{j} \mathcal{C}_{\alpha}\left(K_{j}\right)
$$


Priors leading to well-behaved Coulomb, Riesz gases vs zeroth-order phase transitions

assuming in the case $d=\alpha=2$ that the diameter of $K$ is at most one [49, Thm 5.1.4 a] (then $\mathcal{C}_{\alpha}$ is usually called the Wiener capacity).

Example 6.1. The capacity of a ball $B_{r}$ of radius $r$ centered at $x \in \mathbb{R}^{d}$ is given by $\mathcal{C}_{\alpha}\left(B_{r}(x)\right)=A(d, \alpha) r^{d-\alpha}$ for an explicit constant $A(d, \alpha)$ [42], unless $\alpha=d=2$, in which case $\mathcal{C}_{\alpha}\left(B_{r}(x)\right)=-1 / \log r$. In particular, $\mathcal{C}_{\alpha}\left(B_{r}(x)\right)$ decreases to $0\left(=\mathcal{C}_{\alpha}(\{x\})\right.$ as $r \rightarrow 0$ (which is consistent, as it must with outer continuity).

Similarly, we define the weighted capacity $\mathcal{C}_{\alpha}(K, \phi)$ of a compact weighted set $(K, \phi)$ by replacing the energy $E(\mu)$ with its weighted analog $E_{\phi}(\mu)$. Inner and outer weighted capacities are then defined just as before. It follows from the previous case $\phi=0$ that $S \mapsto \mathcal{C}_{\alpha}(S, \phi)$ satisfies Choquet's axioms on bounded Borel sets, for any given continuous function $\phi$ on $\mathbb{R}^{d}$.

Next recall that if $\mu$ is supported on a compact set $K$, then a Borel subset $C$ of $K$ is said to be a $\mu$-carrier if $\mu(C)=\mu(K)$.

Proposition 6.2. Assume that $\alpha \leq 2$. Then $\mu$ is determining for a regular compact weighted set $(K, \phi)$ iff for any $\mu$-carrier $C$ of $K$ which is the union of increasing compact subsets of $K$,

$$
\mathcal{C}_{\alpha}(C, \phi)=\mathcal{C}_{\alpha}(K, \phi)
$$

Proof. Assume first that $\mu$ is determining and let $C$ be a $\mu$-carrier $C$, which is the union of increasing compact subsets of $K_{i}$ of $K$. Since $\mu$ is assumed determining we have $P_{C} \phi=P_{K} \phi$ and by the outer continuity of $\mathcal{C}$ we have $\mathcal{C}_{\alpha}\left(K_{j}, \phi\right) \rightarrow \mathcal{C}_{\alpha}(C, \phi)$. But, by Prop 2.6 and its proof) $P_{K_{i}} \phi$ decreases to $P_{C} \phi$. As a consequence, $\mathcal{C}_{\alpha}\left(K_{j}, \phi\right)^{-1}:=$ $E\left(\Delta\left(P_{K_{i}} \phi\right)\right) \rightarrow E\left(\Delta\left(P_{C} \phi\right)\right)=E\left(\Delta\left(P_{K} \phi\right)\right)=: \mathcal{C}_{\alpha}(K, \phi)^{-1}$. Hence, (6.2) holds. Conversely, assume that (6.2) holds for any carrier $C$ as above. By Lemma 3.8 (and its proof) there exists such a carrier $C$ with the property that $P_{C} \phi=P_{\mu} \phi$. Hence, by the previous argument $E\left(\Delta\left(P_{\mu} \phi\right)\right)$ is equal to the infimum of $E_{\phi}$ on $\mathcal{P}(K)$. This means, by uniqueness of minimizers, that $\Delta\left(P_{\mu} \phi\right)=\Delta\left(P_{K} \phi\right)$ and hence there exists a constant $c$ such that $P_{C} \phi+c=P_{K} \phi$. But, then it follows from Lemma 3.8 that $c=\sup _{\mu}\left(P_{K} \phi-\phi\right)$ and since $P_{K} \phi$ is continuous (by Lemma 2.13) this means that $c=\sup _{K}\left(P_{K} \phi-\phi\right)=0$. Hence, $P_{\mu} \phi=P_{K} \phi$, which implies that $\mu$ is determining for $(K, \phi)$ (just as in the proof of Theorem 3.10).

Remark 6.3. The capacity criterion above goes back to Ullman in the case when $d=\alpha=2$ and $\phi=0$ (see [56, Thm 2]) and is usually called Ullman's criterion in the theory of orthogonal polynomials on the real line [55].

\subsection{Explicit construction of the measures in Corollary 1.4 and Theorem 4.12}

Following Totik's example for $K=[-1,1]$ (reported in [17]) and the general discussion in [17], the idea of the construction is to start with a sufficiently dense set of points on $K$ and then replacing them by balls of sufficiently small radius, ensuring that the corresponding measure $\mu_{0}$ is carried by a measure which has small capacity. Since it requires no more effort we will consider the general setting in $\mathbb{R}^{d}$ and the Riesz gas with $\alpha \leq 2$, using the general notion of Bernstein-Markov inequalities (definition 4.5).

Lemma 6.4. Let $K$ be a compact domain in $\mathbb{R}^{d}$ and fix $\alpha \leq 2$. For any $\phi \in \mathcal{C}(K)$, there exists a measure $\mu_{0}$ with support $K$ such that $\mu_{0}$ is absolutely continuous wrt $d x$ and satisfies the strong Bernstein-Markov property, but $\mu_{0}$ is not determining for $(K, \phi)$.

Proof. We will use the following sufficient criterion for a measure $\mu$ whose support $K$ is assumed locally regular to have the strong Bernstein-Markov-property: there exists 
Priors leading to well-behaved Coulomb, Riesz gases vs zeroth-order phase transitions

$r_{0}, a, C>0$ such that for any $z \in K$ and $r \in\left[0, r_{0}\right]$

$$
\mu\left(B_{R}(z)\right) \geq C r^{a}
$$

(see [17] for the case $d=\alpha=2$ and [18] for the case of a general $\alpha$ ). This will be contrasted with the capacity criterion in Prop 6.2. Fix a positive integer $k$ and consider the "grid" $K \cap\left(\mathbb{Z} k^{-1}\right)^{d}$. We let $\Lambda_{k}$ be the finite set contained in the interior of $K$ obtained by removing from $K \cap\left(\mathbb{Z} k^{-1}\right)^{d}$ all points with distance less than $k^{-1}$ to $\partial K$ and denote by $\nu_{k}$ the empirical measure on $\Lambda_{k}$. Next take a sequence $\lambda_{k}$ with polynomial decay such that $\sum_{k=1}^{\infty} \lambda_{k}<\infty$, say $\lambda_{k}=k^{-2}$, and set

$$
\nu=\sum_{k=1}^{\infty} \lambda_{k} \nu_{k}
$$

Then, for $k$ sufficiently large the mass criterion (6.3) is satisfied. Indeed, if $k^{-1} \leq 10 r$ say, then (6.3) holds for $\mu_{k}$ with with $a=d$ and a constant $C$ independent of $k$. Hence,

$$
\nu\left(B_{R}(z)\right) \geq \sum_{k^{-1} \leq 10 r} \lambda_{k} \nu_{k} \geq C r^{d} \sum_{k^{-1} \leq 10 r} \lambda_{k} \geq C^{\prime} r^{d+1},
$$

showing that $\nu$ satisfies the mass criterion (6.3). Next, we will modify the construction to get a measure $\mu$ not charging polar subsets. To this end fix a sequence $\epsilon_{k}$ of positive numbers such that $\epsilon_{k}<k^{-1}$ and define $\mu_{k}$ as the measure obtained by replacing each Dirac mass at a point $x$ in the definition of $\nu_{k}$ by the normalized Lebesgue measure on a ball or radius $\epsilon_{k}$, centered at $x$. Equivalently, this means that

$$
\mu_{k}:=\int_{|s| \leq \epsilon_{k}}\left(T_{s}\right)_{*} \nu_{k} d s
$$

where $T_{s}$ denote the translation map $x \mapsto x+s$ for a given $s \in \mathbb{R}^{d}$. We then define $\mu$ as in the decomposition in (6.4). By translation invariance the same estimate (6.5) holds for $\mu$ and hence $\mu$ satisfies the strong Bernstein-Markov-inequality, according to the mass criterion (6.3). Moreover, since $\mu_{k}$ is absolutely continuous wrt Lebesgue measure so is $\mu$. Hence, $\mu$ does not charge polar subsets and clearly has support $K$. Finally, we note that if $\epsilon_{k}$ is sufficiently small, then the capacity criterion (6.2) is not satisfied and hence $\mu$ is not determining for $(K, \phi)$. Indeed, by construction, the set

$$
C:=\bigcup_{k} B_{\epsilon_{k}}\left(\Lambda_{k}\right)
$$

is a carrier for $\mu$, where $B_{\epsilon_{k}}\left(\Lambda_{k}\right)$ denotes an $\epsilon_{k}$-neighborhood of $\Lambda_{k}$, i.e a disjoint union of $M_{k}$ balls in $K$ of radius $\epsilon_{k}$, where $M_{k} \sim k^{d}$. After a harmless scaling we may as well assume that the diameter of $K$ is equal to one. Then, as recalled in the previous section, $\mathcal{C}_{\alpha}$ is sub-additive and invariant under translations. Hence,

$$
\mathcal{C}_{\alpha}(C) \leq \sum_{k} M_{k} \mathcal{C}_{\alpha}\left(B_{\epsilon_{k}}\right) \leq \sum_{k} C k^{d} \mathcal{C}_{\alpha}\left(B_{\epsilon_{k}}\right)
$$

where $B_{\epsilon_{k}}$ the closed ball of radius $\epsilon_{k}$ centered at 0 . Since $\epsilon \mapsto \mathcal{C}_{\alpha}\left(B_{\epsilon}\right)$ strictly decreases to 0 as $\epsilon \rightarrow 0$ (see Example 6.1) this means that, given a continuous function $\phi$ on $\mathbb{R}^{d}$ and a positive number $\delta>0$ we can take $\epsilon_{k}$ sufficiently small to ensure that $\mathcal{C}_{\alpha}(C, \phi)<\delta$. In particular, taking $\delta=\mathcal{C}_{\alpha}(K, \phi)$ the capacity criterion (6.2) is violated and hence $\nu$ is not determining for $(K, \phi)$.

A similar construction yields the following 
Priors leading to well-behaved Coulomb, Riesz gases vs zeroth-order phase transitions

Lemma 6.5. There exists a measure $\mu_{0}$ with support $[0,1]$ such that $\mu_{0}$ is absolutely continuous wrt $d x$, but which does not satisfy the Bernstein-Markov-inequality.

Proof. We recall the following necessary condition for a measure $\mu_{0}$ on $[0,1]$ to satisfy the Bernstein-Markov-inequality [55, Thm 4.2.8]: for any $\eta>0$

$$
\lim _{r \rightarrow \infty} \mathcal{C}_{\alpha}\left(\left\{x \in[0,1]: \mu\left(B_{r}(x)\right) \geq e^{-\eta r^{-1}}\right\}\right)=\mathcal{C}_{\alpha}([0,1])
$$

Denote by $k$ an integer of the form $k=2^{m}$ for some positive integer $m$. We will show that the necessary condition above is not satisfied for a measure of the form

$$
\mu=\sum_{k} \lambda_{k} \mu_{k}
$$

as defined in the previous construction, if $\epsilon_{k}$ and $\lambda_{k}$ are both taken sufficiently small. More precisely, we will show that this happens if $\lambda_{k}=e^{-\epsilon_{k}^{-2}}$ and $\epsilon_{k}$ satisfies

$$
k \mathcal{C}_{\alpha}\left(B_{2 \epsilon_{k}}\right)<\mathcal{C}_{\alpha}([0,1]) / 2,
$$

say. To see this fix $r>0$ and first note that for any $x \in[0,1]$

$$
\left(\sum_{k: \epsilon_{k} \leq r} \lambda_{k} \mu_{k}\right)\left(B_{r}(x)\right) \leq C e^{-r^{-2} / 2}
$$

Next, consider the set $A_{k}$ defined as an $\epsilon_{k}$-neighborhood of the support $B_{\epsilon_{k}}\left(\Lambda_{k}\right)$ of $\mu_{k}$ (which contains the support of $\mu_{j}$ for $j \leq k$ ), i.e. $A_{k}=B_{2 \epsilon_{k}}\left(\Lambda_{k}\right)$. The definition is made so that, if $r<\epsilon_{k}$ then the $r$-neighborhood of $\left([0,1]-A_{k}\right)$ does not intersect $B_{\epsilon_{k}}\left(\Lambda_{k}\right)$. Hence,

$$
x \in\left([0,1]-A_{k}\right) \Longrightarrow\left(\sum_{k: \epsilon_{k}>r} \lambda_{k} \mu_{k}\right)\left(B_{r}(x)\right)=0,
$$

which, combined with (6.7), means that the inequality in condition (6.6) fails when $x \in[0,1]-A_{k}$. But if $\epsilon_{k}$ is sufficiently small, then we get, by the sub-additive of the capacity (just as in the previous construction) that

$$
\mathcal{C}_{\alpha}\left(A_{k}\right)<\mathcal{C}_{\alpha}([0,1]) / 2
$$

say, for all $k$. Hence the capacity condition (6.2) is violated, showing that $\mu$ does not satisfy the Bernstein-Markov-inequality.

\section{References}

[1] R. Allez, J. P. Bouchaud, A. Guionnet, Invariant beta-ensembles and the Gauss-Wigner crossover. Phys. Rev. Lett.109, 094102 (2012).

[2] S.Armstrong, S.Serfaty: Thermal approximation of the equilibrium measure and obstacle problem. arXiv:1912.13018

[3] J. Barré, F. Bouchet, T. Dauxois, and S. Ruffo. Large deviation techniques applied to systems withlong-range interactions.J. Stat. Phys, 119(3-4):677-713, 2005 MR2151219

[4] G. Ben Arous, A. Guionnet, Large deviations for Wigner's law and Voiculescu's noncommutative entropy, Probab. Theory Related Fields 108 (1997), no. 4, 517-542. MR1465640

[5] Berman, R.J: Determinantal point processes and fermions on complex manifolds: Large deviations and Bosonization. Comm. in Math. Phys. Volume 327, Issue 1 (2014), Page 1-47 MR3177931 
Priors leading to well-behaved Coulomb, Riesz gases vs zeroth-order phase transitions

[6] Berman, R.J: Large deviations for Gibbs measures with singular Hamiltonians and emergence of Kähler-Einstein metrics. Communications in Math. Phys. Volume 354, Issue 3, pp 1133-1172 (2017) MR3668617

[7] Berman, R.J: On large deviations for Gibbs measures, mean energy and Gamma convergence. Constructive Approximation. Volume 48, Issue 1 (2018) pp 3-30 MR3825945

[8] Berman, R.J: From Monge-Ampere equations to envelopes and geodesic rays in the zero temperature limit. Math Z. First Online: 02 June 2018. arXiv:1307.3008. MR3936074

[9] Berman, R.J: Statistical Mechanics of Interpolation nodes, Pluripotential theory and Complex Geometry. Annales Polonici Mathematici 123 (2019), 71-153 1810.06939 MR4025011

[10] Berman, R.J: Sharp deviation inequalities for the 2D Coulomb gas and Quantum hall states, I. arXiv:1906.08529

[11] Berman, R.J.; Boucksom, S: Growth of balls of holomorphic sections and energy at equilibrium. Invent. Math. Vol. 181, Issue 2 (2010), p. 337 MR2657428

[12] Berman, R.J.; Boucksom, S; Witt Nyström, D: Fekete points and convergence towards equilibrium measures on complex manifolds. Acta Math. Vol. 207, Issue 1 (2011), 1-27 MR2863909

[13] Berman, R.J; Boucksom, S; Guedj,V; Zeriahi: A variational approach to complex MongeAmpere equations. Publications math. de l'IHÉS (2012): 1-67, November 14, 2012 MR3090260

[14] Biskup, M; Chayes, L: Rigorous analysis of discontinuous phase transitions via mean-field bounds. Comm. Math. Phys. 238 (2003), no. 1-2, 53-93. MR1989669

[15] T. Bloom and N. Levenberg, Asymptotics for Christoffel functions of planar measures, Journal d'Analyse Math., 106 (2008), 353-371. MR2448990

[16] T. Bloom and N. Levenberg, Pluripotential energy and large deviation, Indiana Univ. Math. J., 62 (2013), no. 2, 523-5 MR3158519

[17] Bloom, T; Levenberg, N;Piazzon, F; Wielonsky, F: Bernstein-Markov: a survey. Dolomites Research Notes on Approximation. Special Issue for the "10 years of the Padua points", Volume $8 \cdot 2015 \cdot$ Pages 75-91 MR3437486

[18] Tom Bloom, Norman Levenberg, Franck Wielonsky: A large deviation principle for weighted Riesz interactions. Constructive Approximation volume 47, pages 119-140(2018). MR3742812

[19] R. M. Blumenthal, R. K. Getoor and D. B. Ray: On the Distribution of First Hits for the Symmetric Stable Processes. Transactions of the American Mathematical Society, Vol. 99, No. 3 (Jun., 1961), pp. 540-554 MR0126885

[20] Bogdan, K et al: Potential analysis of stable processes and its extensions. Lecture Notes in Mathematics, 1980. Springer-Verlag, Berlin, 2009. x+187 pp MR2569321

[21] Braides, A: Gamma-convergence for beginners. Oxford University Press, 2002. MR1968440

[22] Chung, K.L: Probabilistic approach in potential theory to the equilibrium problem Annales de l'institut Fourier, tome 23, n o 3 (1973), p. 313-322 MR0391277

[23] Caglioti.E; Lions, P-L; Marchioro.C; Pulvirenti.M: A special class of stationary flows for two-dimensional Euler equations: a statistical mechanics description. Communications in Mathematical Physics (1992) Volume 143, Number 3, 501-525 MR1145596

[24] Dal Maso, G: An Introduction to Г-Convergence. Birkhäuser, 1993. XIV, 341 p MR1201152

[25] Dembo, A; Zeitouni O: Large deviation techniques and applications. Corrected reprint of the second (1998) edition. Stochastic Modelling and Applied Probability, 38. Springer-Verlag, Berlin, 2010. xvi+396 pp. MR2571413

[26] Chafaï, D; Gozlan, N; Zitt, P-A: First-order global asymptotics for confined particles with singular pair repulsion. Ann. Appl. Probab. 24 (2014), no. 6, 2371-2413. MR3262506

[27] Dahlberg, B. E. J: Estimates of harmonic measure. Arch. Rational Mech. Anal. 65 (1977), no. 3, 275-288. MR0466593

[28] Dumitriu, I., Edelman, A.: Matrix models for beta ensembles. J. Math. Phys. 43 (11), 5830-5847 (2002) MR1936554 
Priors leading to well-behaved Coulomb, Riesz gases vs zeroth-order phase transitions

[29] Dupuis, P; Laschos, V; Ramanan, K: Large deviations for empirical measures generated by Gibbs measures with singular energy functionals. arXiv:1511.06928

[30] Fitzsimmons, P. J.; Getoor, R. K: A fine domination principle for excessive measures. Math. Z. 207 (1991), no. 1, 137-151. MR1106819

[31] Forrester, P. J.(5-MELB) Log-gases and random matrices. London Mathematical Society Monographs Series, 34. Princeton University Press, Princeton, NJ, 2010 MR2641363

[32] García Zelada, D: A large deviation principle for empirical measures on Polish spaces: Application to singular Gibbs measures on manifolds. Ann. Inst. H. Poincaré Probab. Statist. Vol.55, No. 3 (2019) arXiv:1703.02680 MR4010939

[33] RK Getoor. Some asymptotic formulas involving capacity. Probability Theory and Related Fields, 4(3):248-252, 1965. MR0190988

[34] D.J.Gross; E.Witten: Possible third-order phase transition in the large N lattice gauge theory, Phys. Rev. D, 21, 446-453, (1980).

[35] Guedj,V; Lu, C.H: Zeriahi: Plurisubharmonic envelopes and supersolutions. J. Differential Geom. 113 (2) 273 - 313 (2019). MR4023293

[36] S. Gunasekaran; D.Kubizňák; R.B. Mann: Extended phase space thermodynamics for charged and rotating black holes and Born-Infeld vacuum polarization. J. of High Energy Physics (2012:110).

[37] Ismaeel, T: On the Wiener criterion in higher dimensions. Boundary Value Problems (2017) 2017:1747 MR3736606

[38] K. Johansson, On the fluctuations of eigenvalues of random hermitian matri ces, Duke Math. J., 91, pp.151-204 [1998] MR1487983

[39] K.Johansson, The Longest Increasing Subsequence in a Random Permutation and a Unitary Random Matrix Model, Math. Res. Lett., 5, no.1-2, 63-82, (1998). MR1618351

[40] Kiessling M.K.H.: Statistical mechanics of classical particles with logarithmic interactions, Comm. Pure Appl. Math. 46 (1993), 27-56. MR1193342

[41] Kiessling, Michael K.-H: Spohn, H: A note on the eigenvalue density of random matrices. Comm. Math. Phys. 199 (1999), no. 3, 683-695 MR1669669

[42] 9] N.S. Landkof, Foundations of Modern Potential Theory, Grundlehren der Math- ematischen Wissenschaften, 1800 Springer-Verlag, Berlin, (1972). MR0350027

[43] Levenberg, N: Monge-Ampère measures associated to extremal plurisubhar-monic functions in $\mathrm{C}^{\wedge}$ N, Trans. Amer. Math. Soc., t. 289 (1), 1985, p. 333-343 MR0779067

[44] Majumdar, S.N; Schehr, G: Top eigenvalue of a random matrix: large deviations and third order phase transition. J. Stat. Mech. Theory Exp. 2014, no. 1, P01012, 31 pp. MR3172178

[45] V. P. Maslov: Zeroth-Order Phase Transitions. Mathematical Notes (2004), Vol. 76, Issue 5-6, pp 697-710 MR2129341

[46] M.L. Mehta, Random Matrices, 3rd ed., Pure and Applied Mathematics, Vol. 142, Elsevier/Academic Press, Amsterdam, 2004. MR2129906

[47] Messer, J; Spohn, H: Statistical mechanics of the isothermal Lane-Emden equation. J. Statist. Phys. 29 (1982), no. 3, 561-578 MR0704588

[48] Nguyen, Q. D., Regularity of certain sets in C n. Ann. Polon. Math., 82 (2003), 219- 232 MR2040807

[49] Ransford, T: Potential theory in the complex plane. (English summary) London Mathematical Society Student Texts, 28. Cambridge University Press, Cambridge, 1995. x+232 MR1334766

[50] M. Riesz, Integrales de Riemann-Liouville et potentiels, Acta. Sci. Math. Szeged. vol. 9 (1938) pp. 1-42.

[51] Saff, E. B. \& Totik, V., Logarithmic Potentials with External Fields. Grundlehren der Mathematischen Wissenschaften, 316. Springer, Berlin-Heidelberg, 1997 MR1485778

[52] Serfaty, S: Systems of Points with Coulomb Interactions. Proceedings of the International Congress of Mathematicians (ICM 2018), pp. 935-977 (2019) arXiv:1712.04095 MR3966749

[53] Simon, B: The Christoffel-Darboux kernel. Perspectives in partial differential equations, harmonic analysis and applications, 295-335, Proc. Sympos. Pure Math., 79, Amer. Math. Soc., Providence, RI, 2008. MR2500498 
Priors leading to well-behaved Coulomb, Riesz gases vs zeroth-order phase transitions

[54] S. M. Stishov: Does the phase transition exist in the one-component plasma model? Jour. Exp. Theor. Phys. Lett, 67(1):90-94, 1998.

[55] Herbert Stahl; V. Totik: General Orthogonal Polynomials. Encyclopedia of Math. Cambridge Univ. Press (1992). MR1163828

[56] Ullman, Joseph L: Orthogonal polynomials for general measures. I. Rational approximation and interpolation (Tampa, Fla., 1983), 524-528, Lecture Notes in Math., 1105, Springer, Berlin, 1984. MR0783300

[57] Zeitouni, O; Zelditch, S: Large deviations of empirical measures of zeros of random polynomials. Int. Math. Res. Not. IMRN 2010, no. 20, 3935-3992. MR2738347

[58] F. Y. Wu. The Potts model. Rev. Mod. Phys. 54, 235 (1982) MR0641370 


\section{Electronic Journal of Probability Electronic Communications in Probability}

\section{Advantages of publishing in EJP-ECP}

- Very high standards

- Free for authors, free for readers

- Quick publication (no backlog)

- Secure publication $\left(\mathrm{LOCKSS}^{1}\right)$

- Easy interface (EJMS²)

\section{Economical model of EJP-ECP}

- Non profit, sponsored by $\mathrm{IMS}^{3}, \mathrm{BS}^{4}$, ProjectEuclid ${ }^{5}$

- Purely electronic

\section{Help keep the journal free and vigorous}

- Donate to the IMS open access fund ${ }^{6}$ (click here to donate!)

- Submit your best articles to EJP-ECP

- Choose EJP-ECP over for-profit journals

\footnotetext{
${ }^{1}$ LOCKSS: Lots of Copies Keep Stuff Safe http://www. lockss.org/

${ }^{2}$ EJMS: Electronic Journal Management System http://www.vtex.lt/en/ejms.html

${ }^{3}$ IMS: Institute of Mathematical Statistics http://www.imstat.org/

${ }^{4}$ BS: Bernoulli Society http://www. bernoulli-society.org/

${ }^{5}$ Project Euclid: https://projecteuclid.org/

${ }^{6}$ IMS Open Access Fund: http://www.imstat.org/publications/open.htm
} 